LYNCH

1. LNCH $20 \mathrm{JWLY}$

AUSTRALIA AUE/988

$$
\begin{aligned}
& \text { AUSTRALIA } \\
& \text { ZO JUN-1 AUO } 1988
\end{aligned}
$$


19 July Bg En Rerte Guildford Kellerberri Grocries 117,00 Lamp 12.00 Left Midland offire of CSIVO at lli 3osm 183,753

Passed turnoff to York at -784 Fuirly heary urodeanbs until n - 815 when rees begin to cuppean someulat when trees begin to dppen farce hean

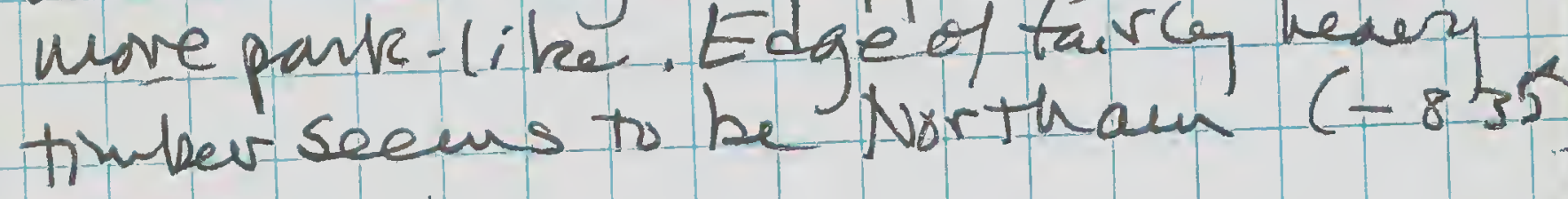
noted Mute Swans and Coots on Avon R. Reached Kellerbersin at 14:00 (990) Gas 30.7 litre $061.99=19.00$

Drove N. to CSIRo house - 9630 So, totel distance from Midland is $210 \mathrm{~km}$ Sow a wedge-taibl eagle cuttin low over Weeds Res. at the Crest 10 Nauken kestres and 3 Blackdiordereb kites beturen Novtham aud Rellerbersin. 
Lotuh C81R0 Kouse N. of Kelluban: cab last uz WT - frost au over car in A.M. 3974.2

9:30- Set up hist net in crea wher 1 heand 2 orer sins. Thr

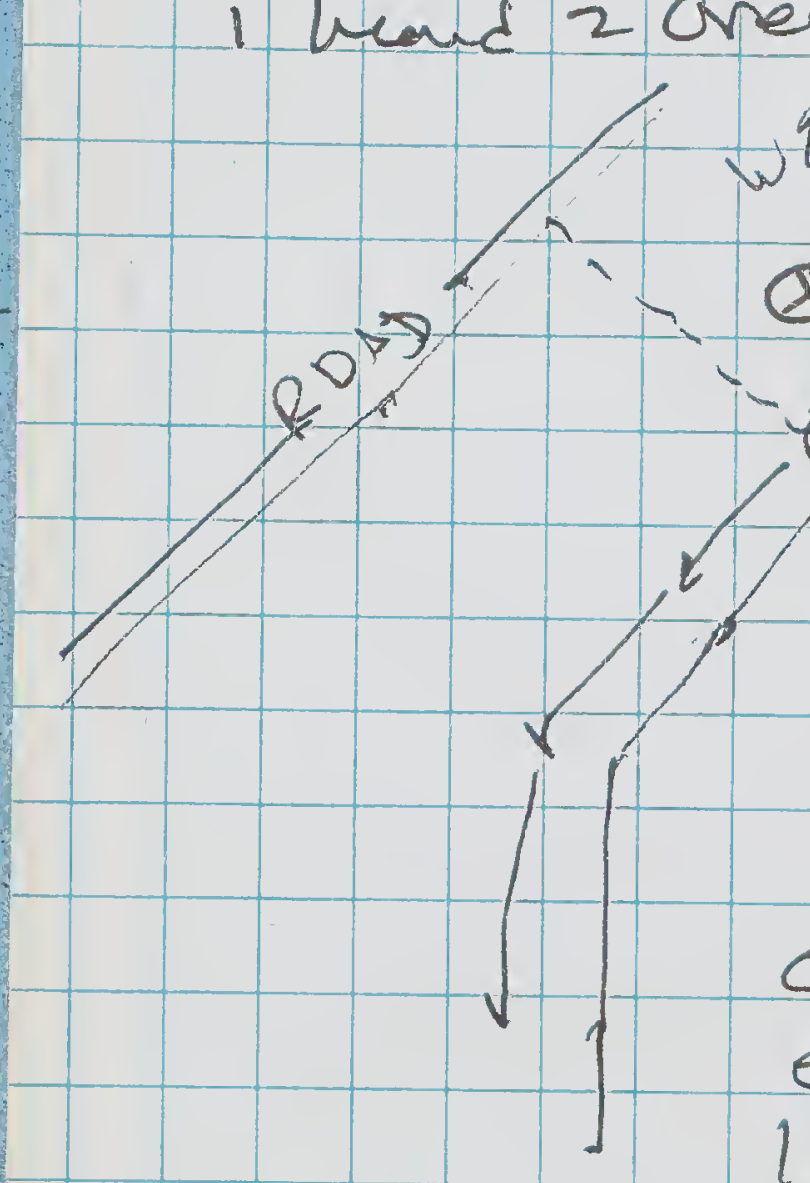
Start strip net Trousects at 10.00 clear,cool, slibrege 15. - walk $25 \mathrm{~m}$ in fromedqe, parallel to it; head sW to el bow in resure bownday, Then on to end of reserve. count all bivdo 252 e.Ther side; stopand listen for 10 Mili ever 5 om (Count This stop w. Plevious I st So: $\sim 10$ Duwoswall over hear strip) OUT3:0 E 25 m (1B) Willie waytail (edge)

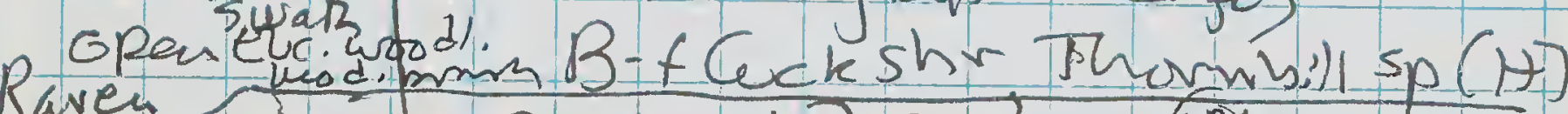
Raven 2nd. Bellbind, 2nd: o(13) chat B 10 PT Line Paw Tree M(Sei) oper Euci woode. ( 9 OH, I P)

PTLine.lar 4R sav. Tree Martio (OL) Treallow

ar 5 hrithr

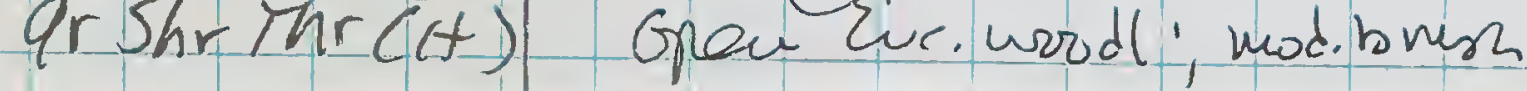

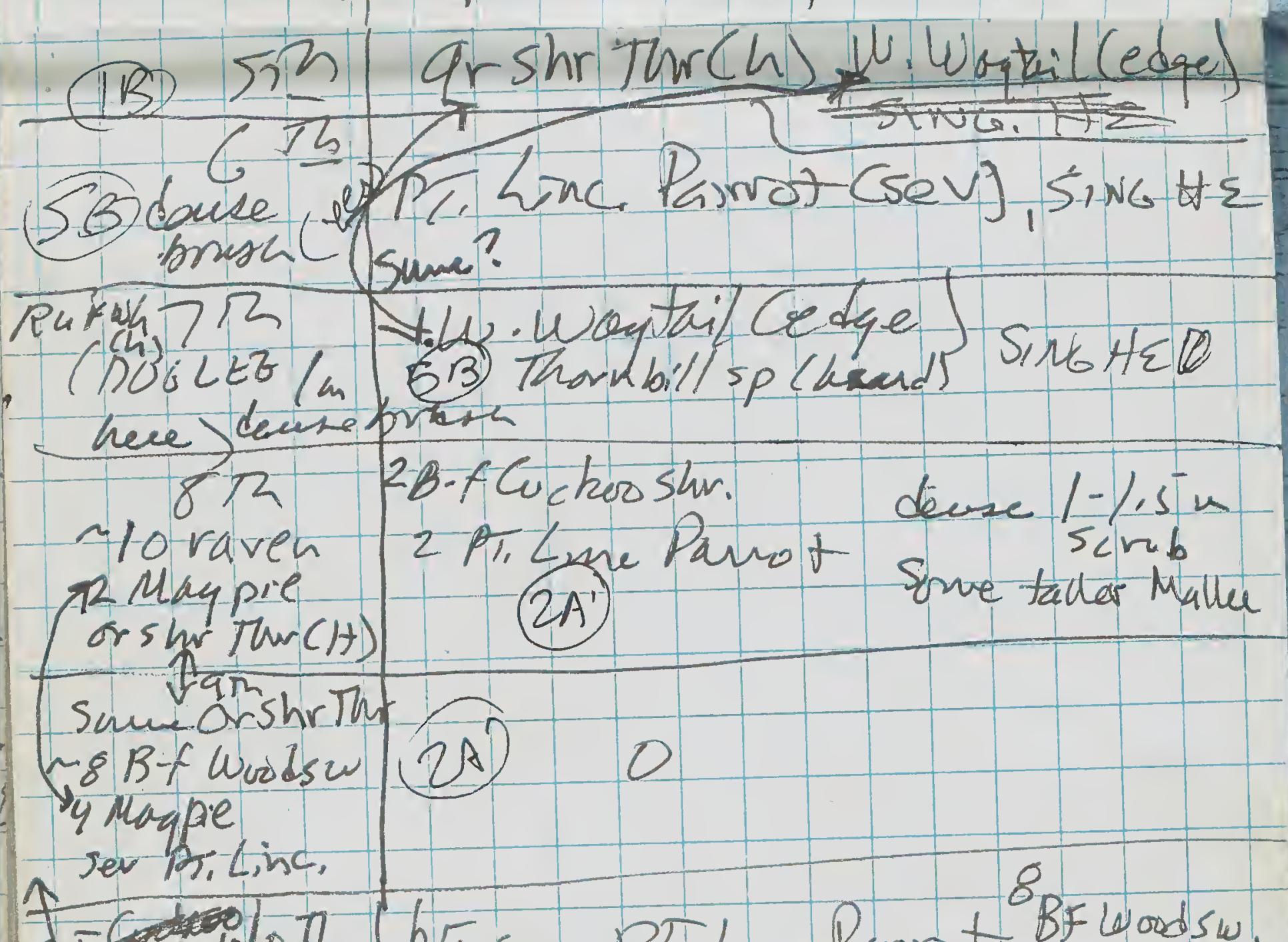

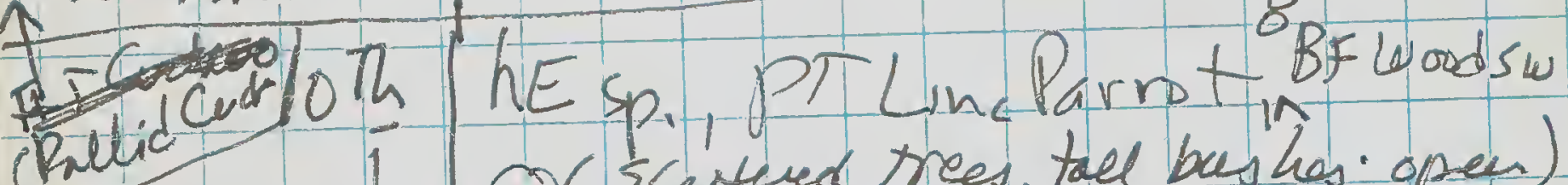
(i) Scalticed trees, tall bashes; open) Bellbird(A) grshrThr(h) 12 SIvG1tE, 2PILinc Pan (OH) 3 Mudlarls cpalbo ac Sov. Pr. Linc. Parr(peacled) (17)

$H$-heard $O H=$ flyingoverhead $P=$ perched $p=$ perched 


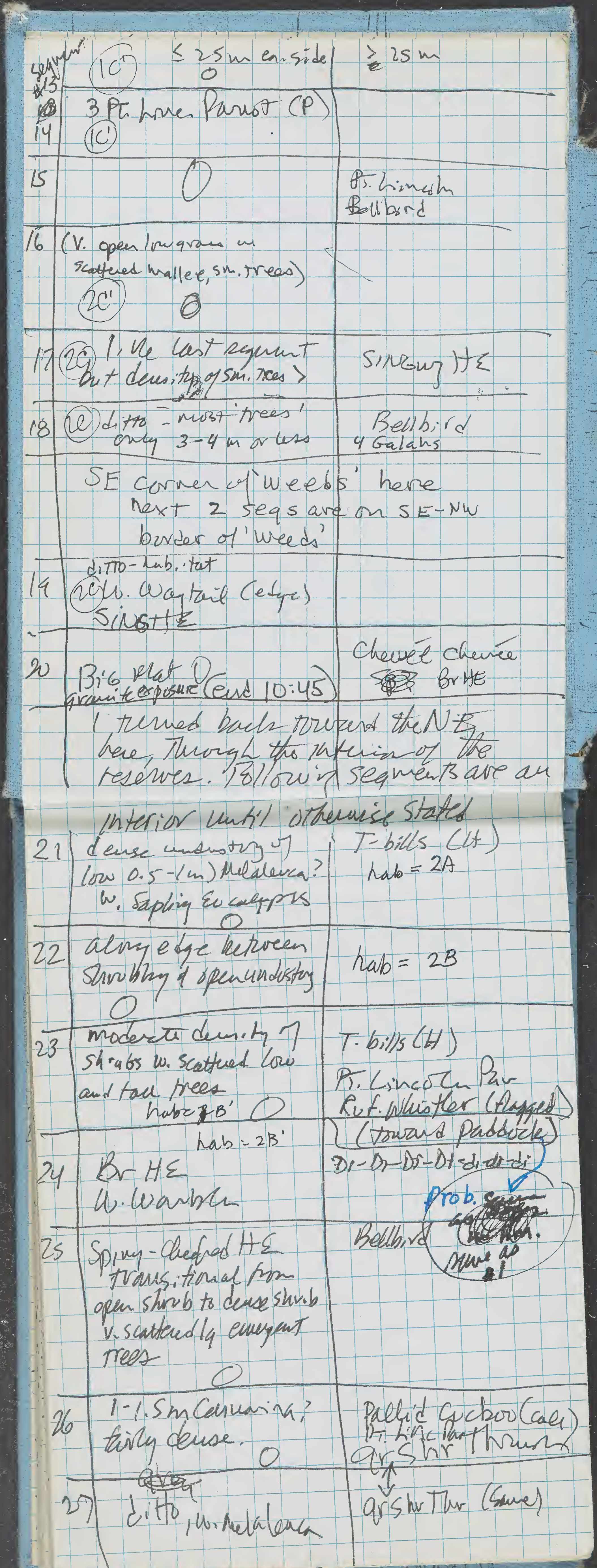


28 deunvielalenca

thichats 0

$-\quad h a v=S B$

29 Melakua-Casuar.

2t m ligh

habiss 0

30 dito 0

Vals $=5 B$

31 Someulat lower

- mune open suab

ve suatued nallec dups

(O) 2BI

32 deuse 1-1.5m surh-lots of yellow Pi.luc Parots

BrHe hav $=\frac{7}{5 c}$

33 area w. mave

PT.Linc Pair (P) $20 \mathrm{~m}$ teee; cas dewne

Tree Maitin OH

Greyfoutail

Votoce

39 ditto but fower shnbs

trav $=I B$

2 ches-r $+b^{2}$

2 (1 cirrmim,wool habs=1B

nest materio 1 )

Singivglt

PT. Linc. Purots

Weebill

39 dithobabitat

2 PTílinc.Pan

$l_{a b}=1 B(P)$

38 open w. Sattered swar brish climpsoand wallep,

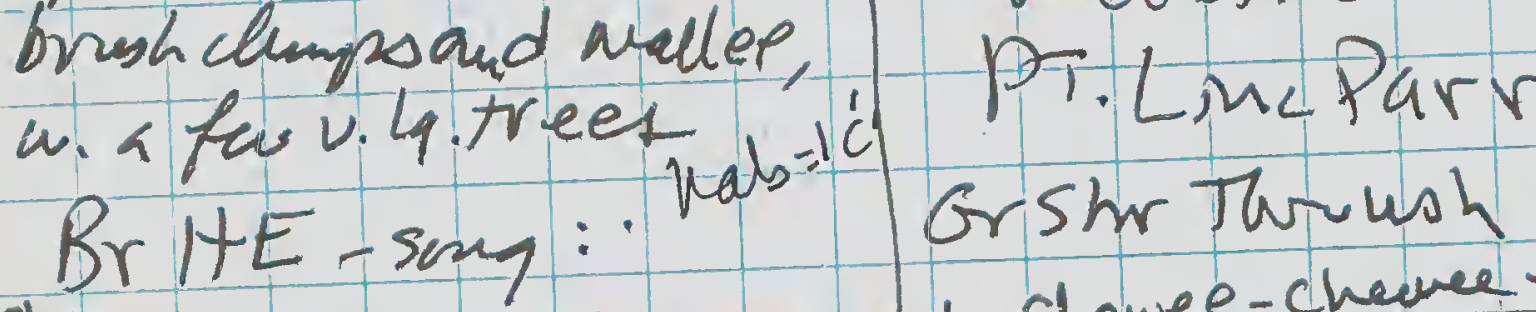

chewrchew-chen. Thet-pzeet-zeet-Chewee-chemee-chare

V.open_los of aran af

36 bave qround - v. Scatperd

14. tree at a few isolated sunobs mab=1 $1 c^{\prime}$ 4 P. Lmc. Pau. (P)

3 N $50 \%$ bove ground or $\mathrm{hav}=1 c^{\circ}$ v. boohabs; fur hurnes a combet lypees 0

(38 dito 0

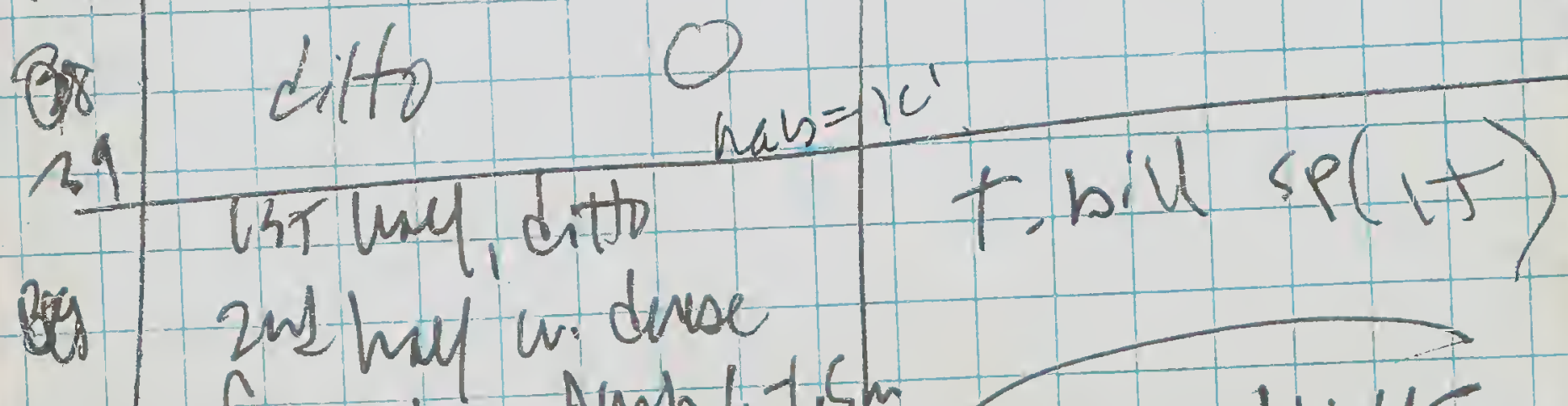

40 Aswaina Amb 1.1.5m ad 11:4S 
li:45 5106106 HE in vet l'd opened Sp. Code 608 earlien. band size 3 Bands: LefT - wh/metal Right - DK.Blue/white $43.5 \mathrm{gm}$ gross ar. the 19.5

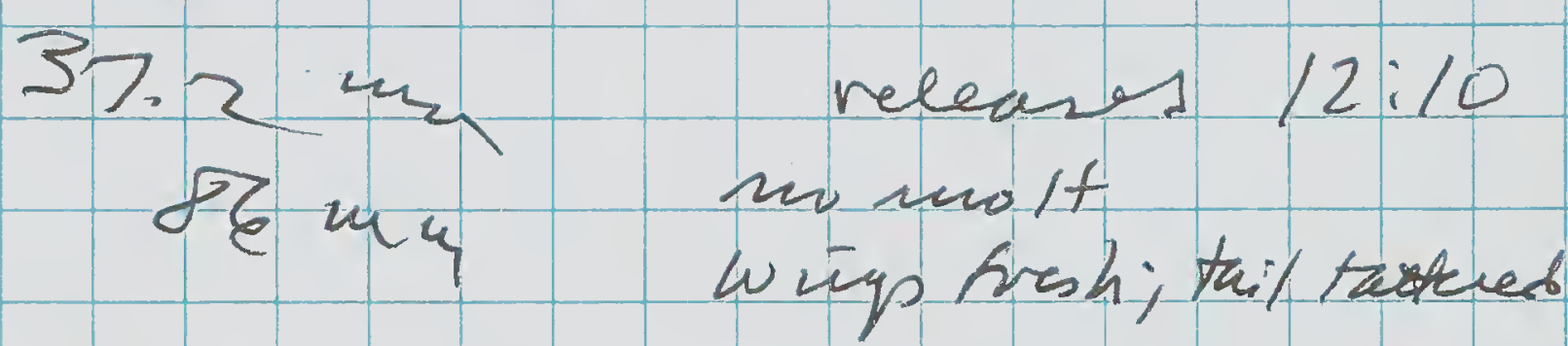

$12: 30$ start awive tranect Ruorgh SW ace of Weeds tion coss road SW Thugh center of Weeds, Then baek

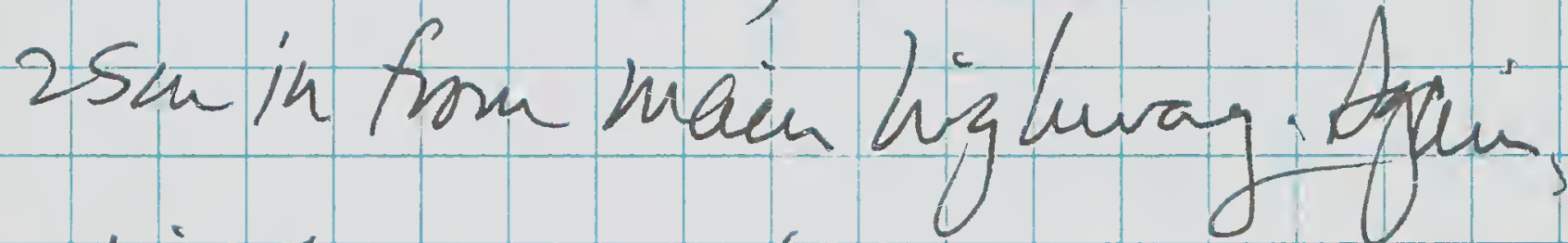
hirds tallicd us som sequents

No) Habitat Type $\leqslant 25 \mathrm{~m}$ hr. obs. I $>25 \mathrm{~m}$ fr. obs. 1. 15r 3/y tal (2.34) GrShr Tarush Casuacinascub; Then - pencu, yave dirr, qrass, scateodlow shrulos Thornbill SPCH

V: opren, low, much

diryshoniclewp of for quins at fareud (IC')

3 open,qlato, tor shnis but pev. Acurtied 19 . glems

2 Miners

2 Pr.cinc(P) Grshring $H)$ Wallie Wagrail(OH) Br HE (H) 101

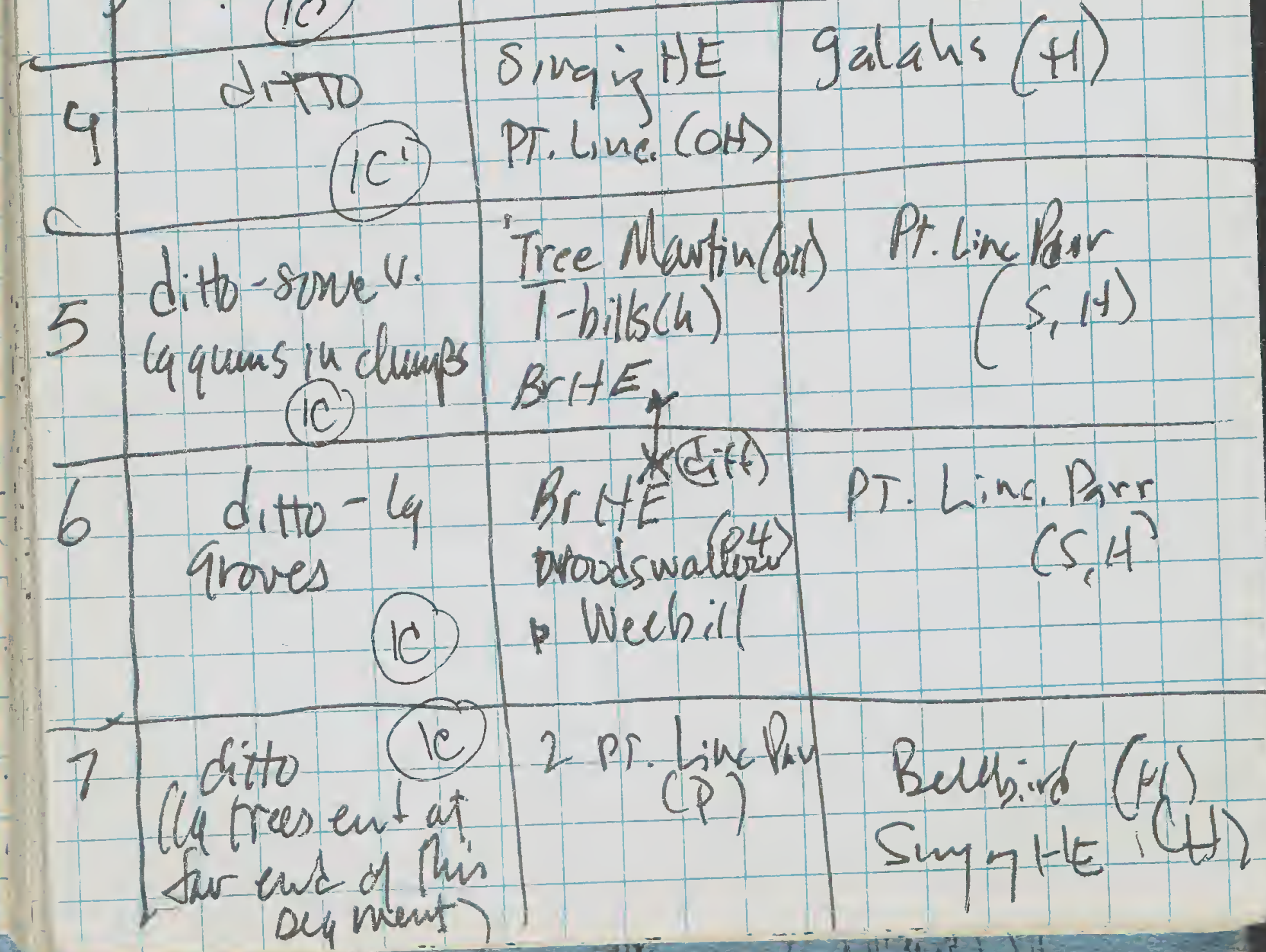




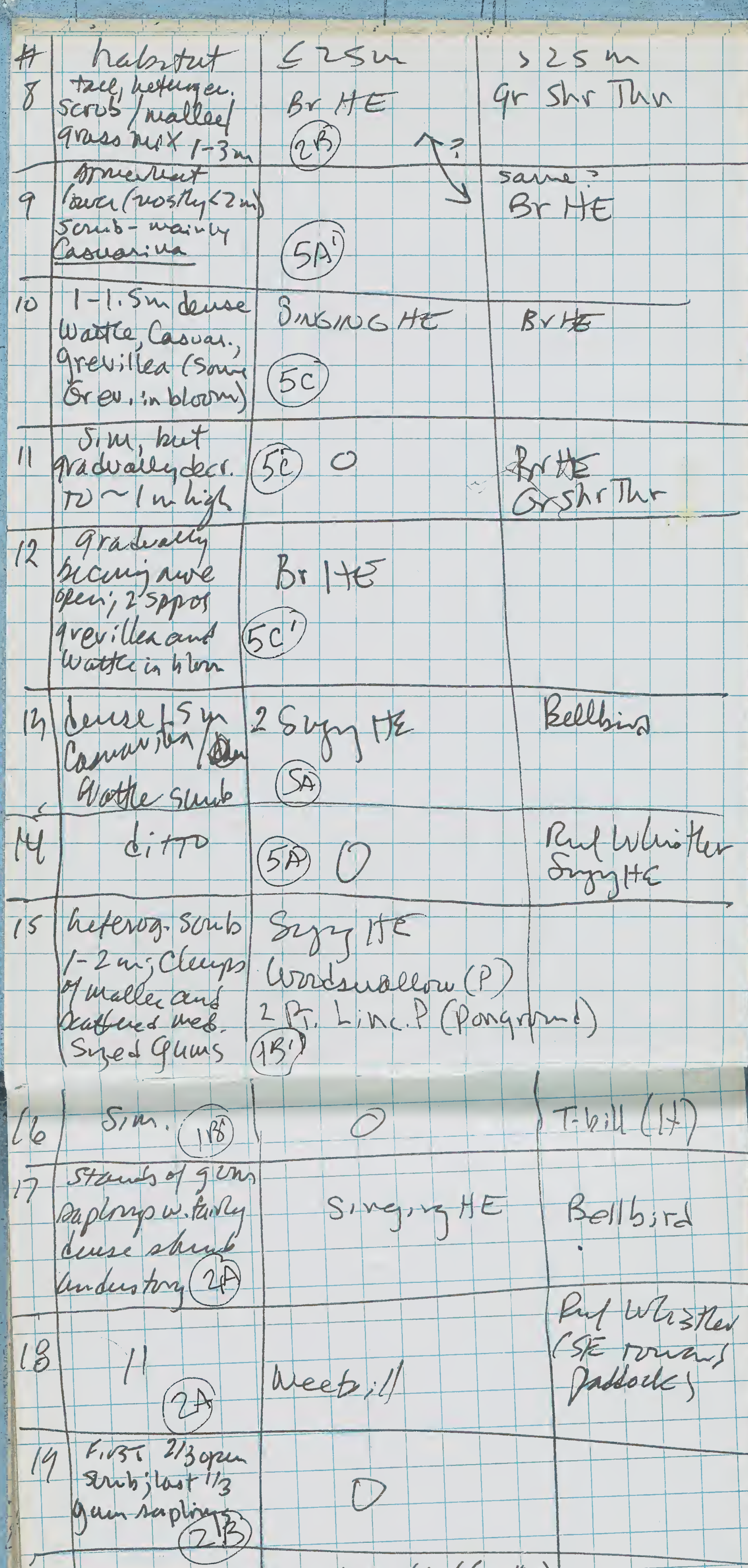
$20 \begin{aligned} & \text { Euc. (2C) } \begin{array}{l}\text { Weebills (difficu"/8) } \\ \text { Miner }\end{array} \\ & \text { suplinip w. }\end{aligned}$ sparse shroubs

21 dewershuh (Mel.?)w. C) Eve.saping Pe. cinciParr Ris Reverent ands - $100 \mathrm{~m}$ firs $S$ houndern 1 reserre- paest serval che 'edqe' sequents 


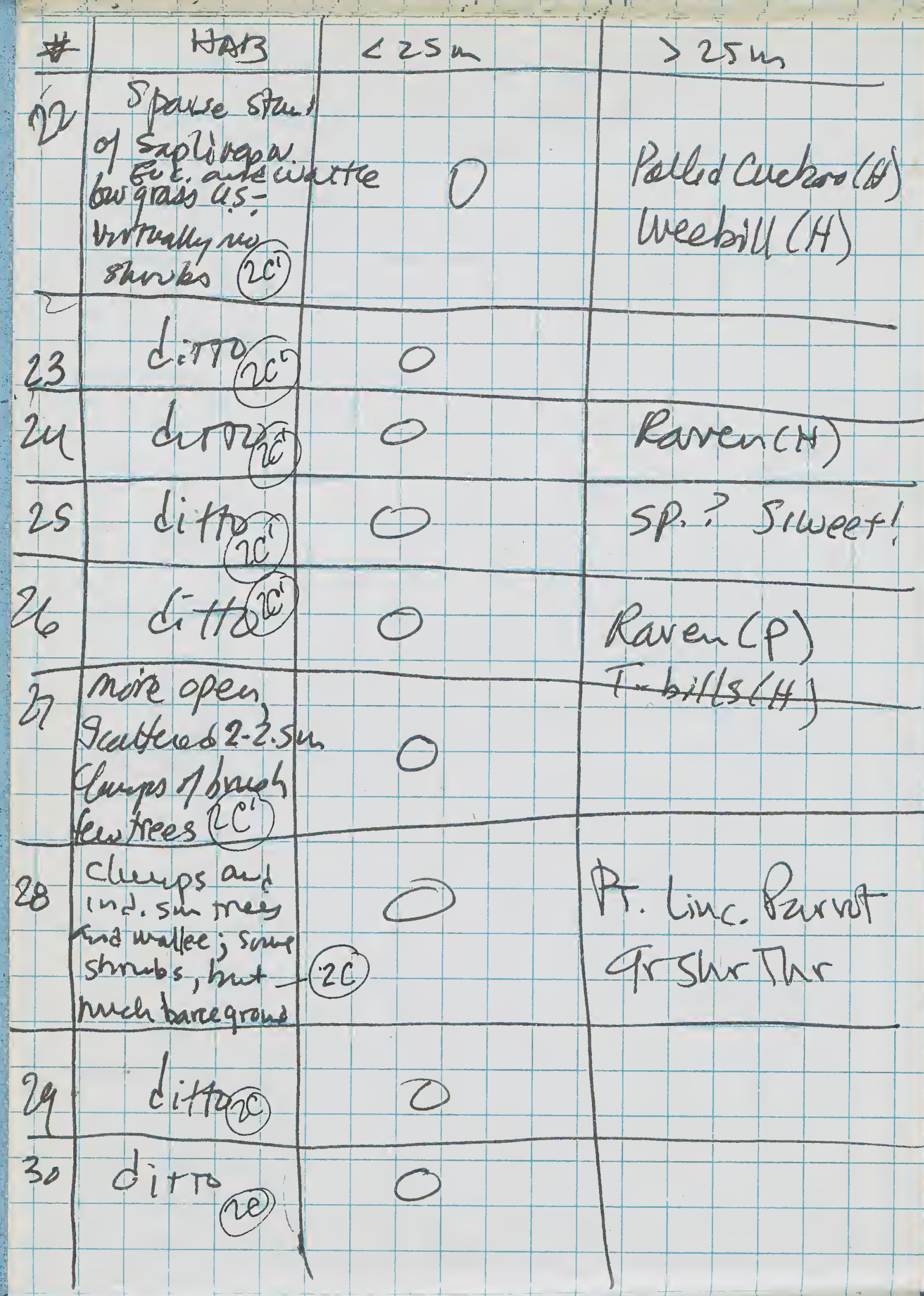

$31 \operatorname{diss}_{20} 0$ Jugzhtte

32 Lot3 of 1-2.5n

tru clumps 1 zweebills PT LincPan serrial bruss 9r Fanta: I pp, tscatted \&chrThombill Ravea (5) suplip a trees qu Warble

up to 15aranda, Ruf whister

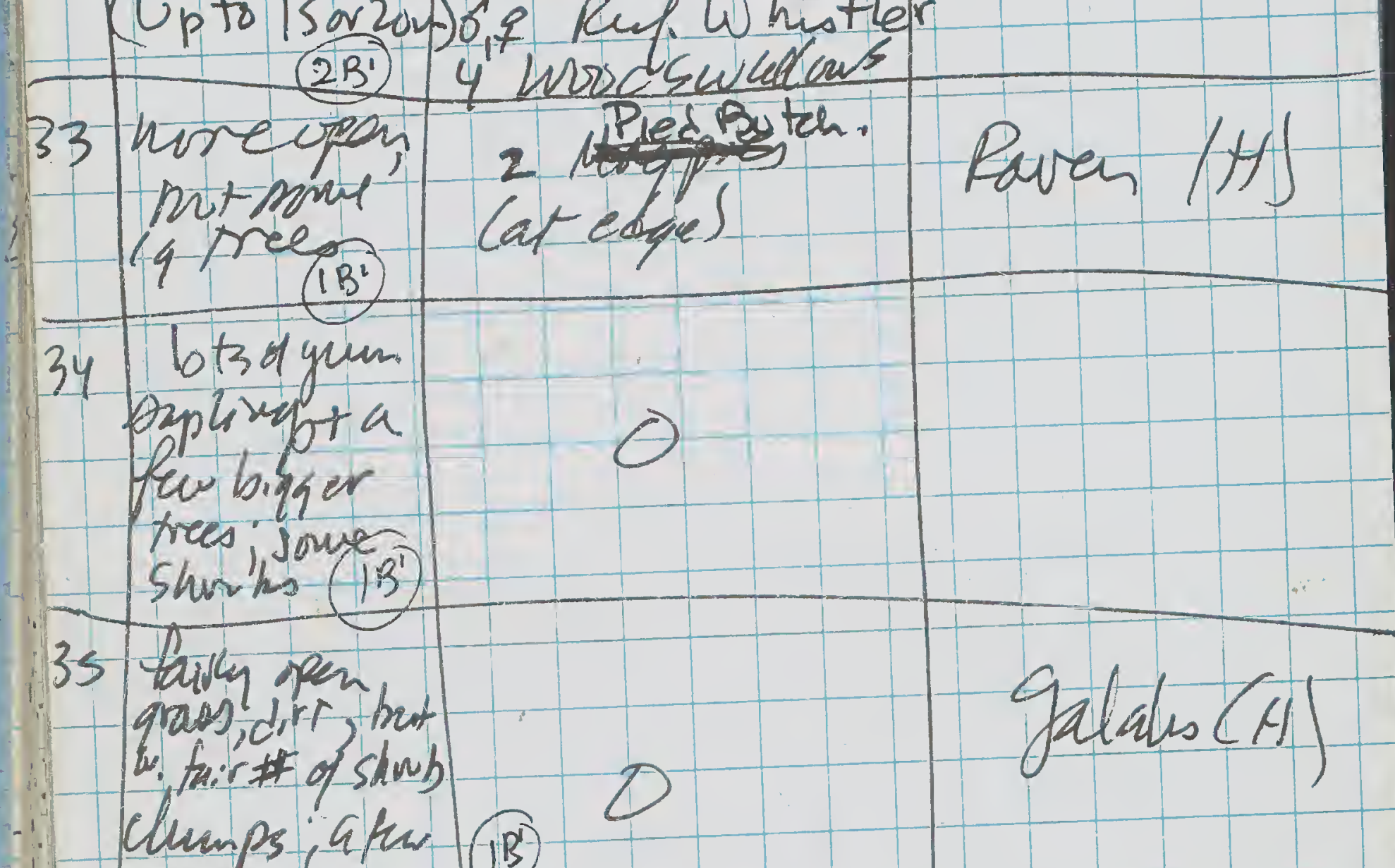

climps; afur

Del gum (B)

36 denre inatte coordowallow(ot)

$1-1 / 2 m$ man

of it in blom DT hinculn (P)

This mis meto swicome a weedd - Tum bauk NE Ravalel rd 


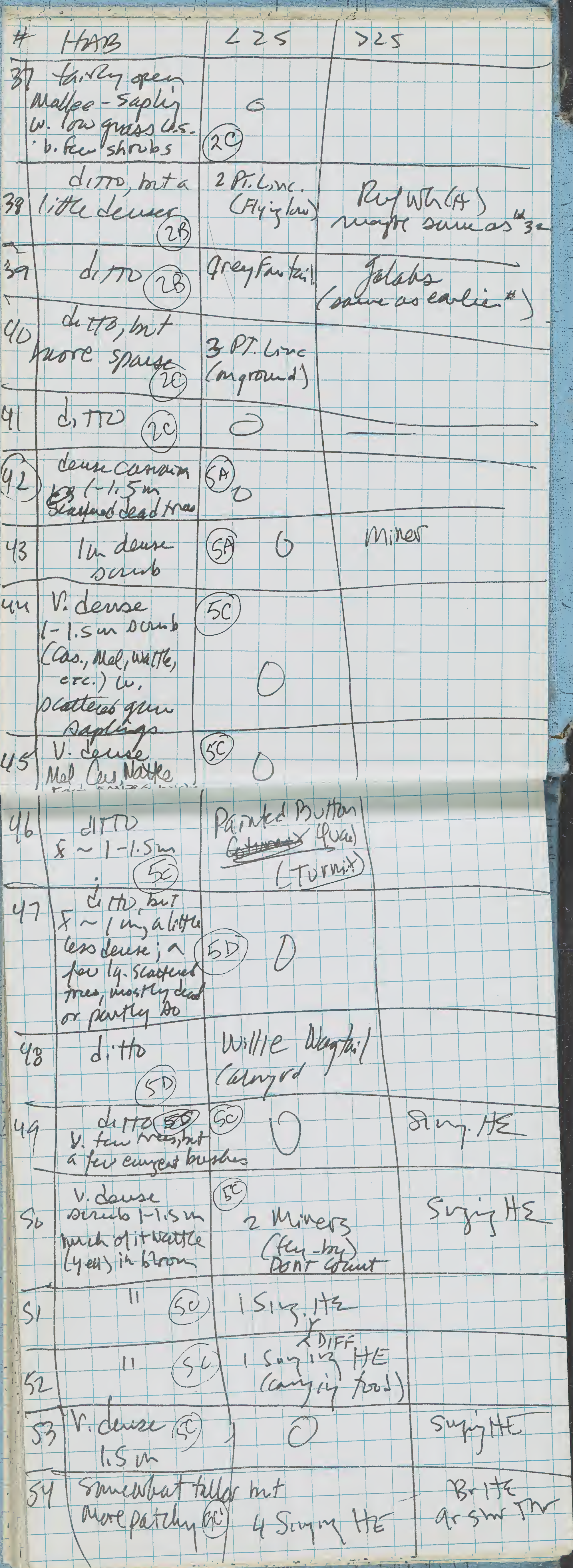


20 July

(contos)

15:20 Pardated fe in saun net That Caught singing HE This A.M. This one doesn't get olor bauded

Code 976 Band Size $\$ 1$ left: White/metal $\$ 014-57663$

9055 WT. 39.5 tare 29.5 HL2/.3 met 10.0 al 66

phant tod,s

Drosera macrantha - sm. climbino sundew now in flower - brantitol single white buttercup-like bloom

Baeckea Alor;bunda - bush w, Tiny scaly leaves, pale puple flower

Hibbertia eatoni:-sim, buy with yellow flower, large hooks

hooks

\section{D's countes}

Judith Harvey Plaut Ecolog:s Dept Cons. car land Mam

Cicerello:s - fresh Jizh in Freemantle ; Fshermais harbor (lokm w. of Perth)

Cloremont shoppria (pr. - Sust w. of Perth - $1 / 2$ way to Freemante

21 July "KWONGAN" or Heath-hand tauts ot Durahompir East 1.D,'s by Judith Harvey arevillea pritzilii - pill.like w. 2 "long mades" gintegrifolia - Shor t "z" nedes" G.exce/sior - V.lni (6") 'vendes" Evcalyphes drummondi: lorw, twisted $\Leftrightarrow$ roinds Casuarina campestris - coum, w. Sm rectaigubar fin. c. acutivalvus - lawer,spinier fruis AsToloma serratofoliam - Thy Ive currec Melatena hou re? leavo radial veins on leaves fr wiret hud.like berries $~$ 
Melaleuea Eleptospernoides smale, pets1-like umspined leares

Leucopogon - tiny-leaved verswn of Astrolowg - minute

Ec deocolea mrnostachys bia bunch-graso losky sedge SED $6 E$ wire-grass ryze leares-smoota life size topured sud thed

Wattes-7and to tell-ove w. ina narrow leva and sin yelloutlown all the Acalia Rige A. nigripilusa spine sross-

Synaphea sp. 1 kow growirg Proteaceres plant w. Brillicunt yellow 'spikes'.

Verticordia (featherflower) Cauliflower-like 2' high

minute ( / / unn) leaves several spp. here vis $Y^{\prime} y^{\prime}=$ Dome un bloon (Lavender or yellow) (.

Davisea hakeoides Rea family Spike-like lleaves'
low-growiy dumps
Trangular tods

Mirbelia spinosa - Moretypical pea-spina - small Yellow flower u. red.or Ceater

Acalia muitispiata - Met 5 ze $(1.5 \mathrm{~m})$ rounded bush w, needle-like lears $12^{\prime \prime}$ "ery and rows of yellow bau-supid florers

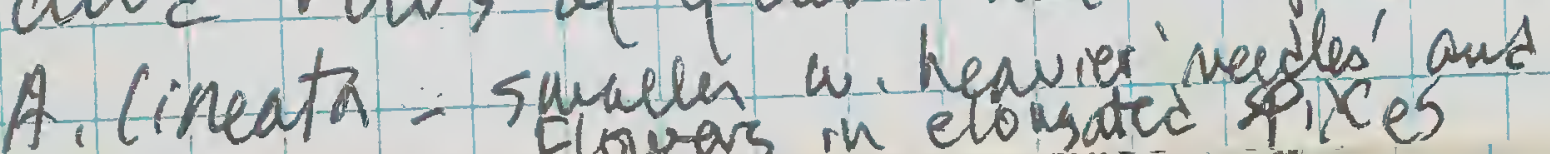


Petrophile Cricifolia - Proteiacurs bas nw. Rersistant furzzy Crulike = Ifs froit - flowen yellow from f) 3 sed elorgate tulip-like folded bud' soler ens

Hahen erecta
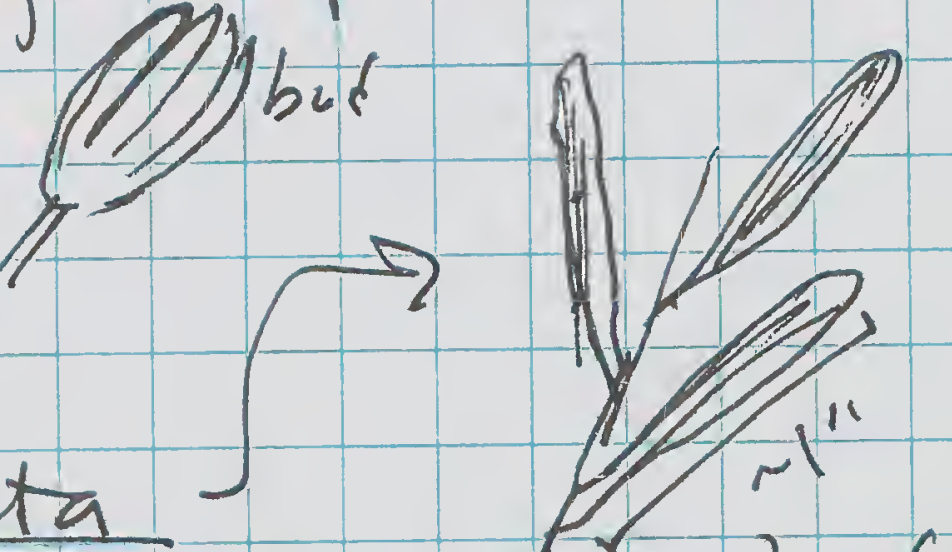
alvayp have persistery asola trit b. sprit-"Clausbee" shape

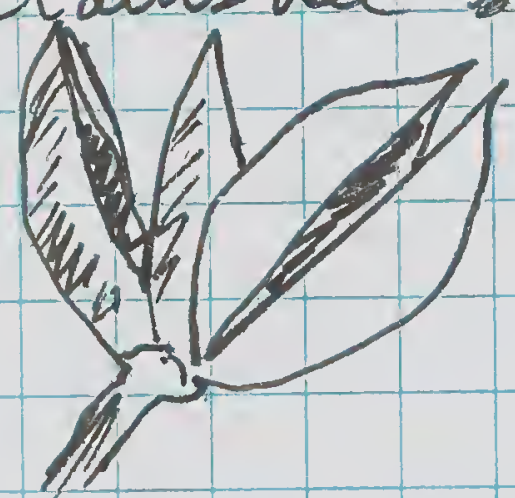

revoluta
Dianellat large lily w.
dead flower

Borya Censtricta - Huy pincusion norewd w. brilliaut white flowers (a'resurrection' plaut)-Lelee Liliaceae

$$
\text { PRoDS E. Durakoppin }
$$

1. Acacia multispilata Yellow popcorm-like flowers 2. Synaphea Sp. - yellow proteacous 3.

4. qrevillea excelsior-pine-liketree 5. Xylomelum anqustatum (Woody Pear)

6. Perrophile cricifolia 7. Borya constricta- clos-ys 8

9. Grevillea ex celsion- flower lyellow Tarae euvergent spike 10. Acacia sp w. Gudith is piture vilory Thim reutle-like leares (-4") bushy tree y un lugh

11. General vilw of 'Kwongan' vegetation 12. Peculiar missilike se dge - gewus anken 13. 'TY Pilal'sedge - Ec deocolea monostachys Mi Davese a hakeoides - wered pea 
Sollya heterophylla - Soft leared bush-a. elnzate I"leaf clastars<smiles>C#CC1CC1CC1CC1</smiles>
blueflowers, 1/2" quen pods.

sopogon scabriosus

lovg, s tout heedled like leaves with beats fue starbust flower in lavandr horn in cluster at

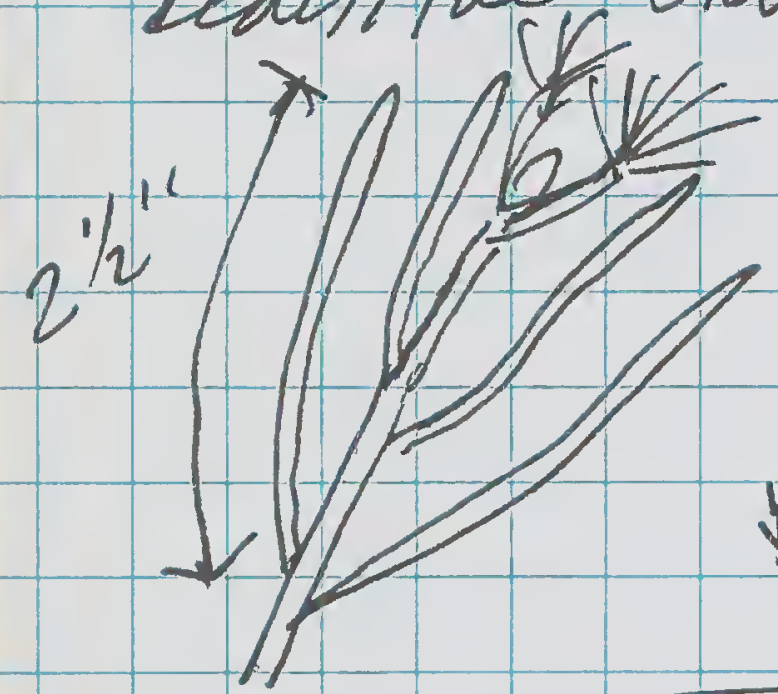
$b$ ranch tip. Reis istant worde glubase 'pineapple troit on branchatils somerbat fazzy

Dryandra amata - exagevated evarzinate bolly-Aype lewsos

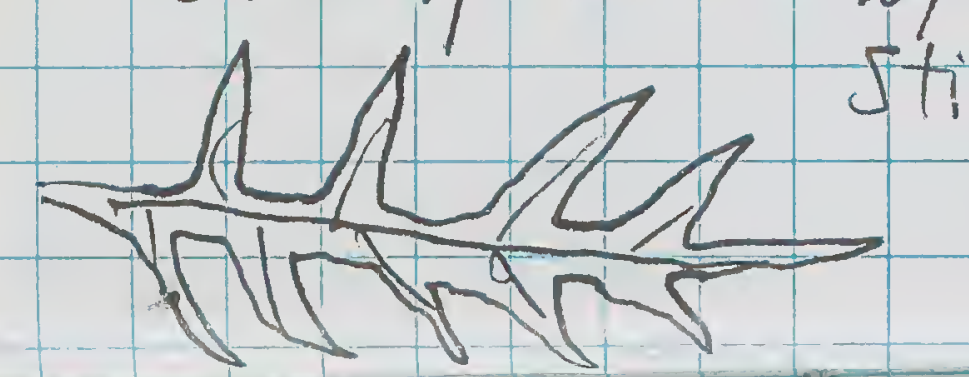

stiffileatheryloures oligophylla

Dampieva $\pi$ blue flarer in Leschervalta tamily . Caves cylindrical photos-contd

14. moss-like sedge w. 'nawal one in bochquound

15. Isopogon seabriosus - Plover

16. fruits of Casuationa acutivalvus

17. Dampiera - brique blue feowers

18. Daresea weird pea lu. Heedle. Whe lewes

19. Orevillea pritzilii

20. Dampiera flowers $\omega_{\text {. }}$ $\frac{\text { Hibbertia }}{\text { (hooked equanis) }}$ (yellow)

$$
\text { (END OF Roll \#1) }
$$

A. Stenoptera - Jam-1ibe Acacis w. $\frac{\text { stout heavily veined elnigate }}{4}$ leaves

Casuariua huegeliana-r.tau Apecies - open tree form 
Lepto spermum arabesceus (Ten Tree) - opnght open powth form - baves orate, clustered alpy huizo-not spina- open white 5-pets led flosa /like a strawbiny) 1/4" in dianoter

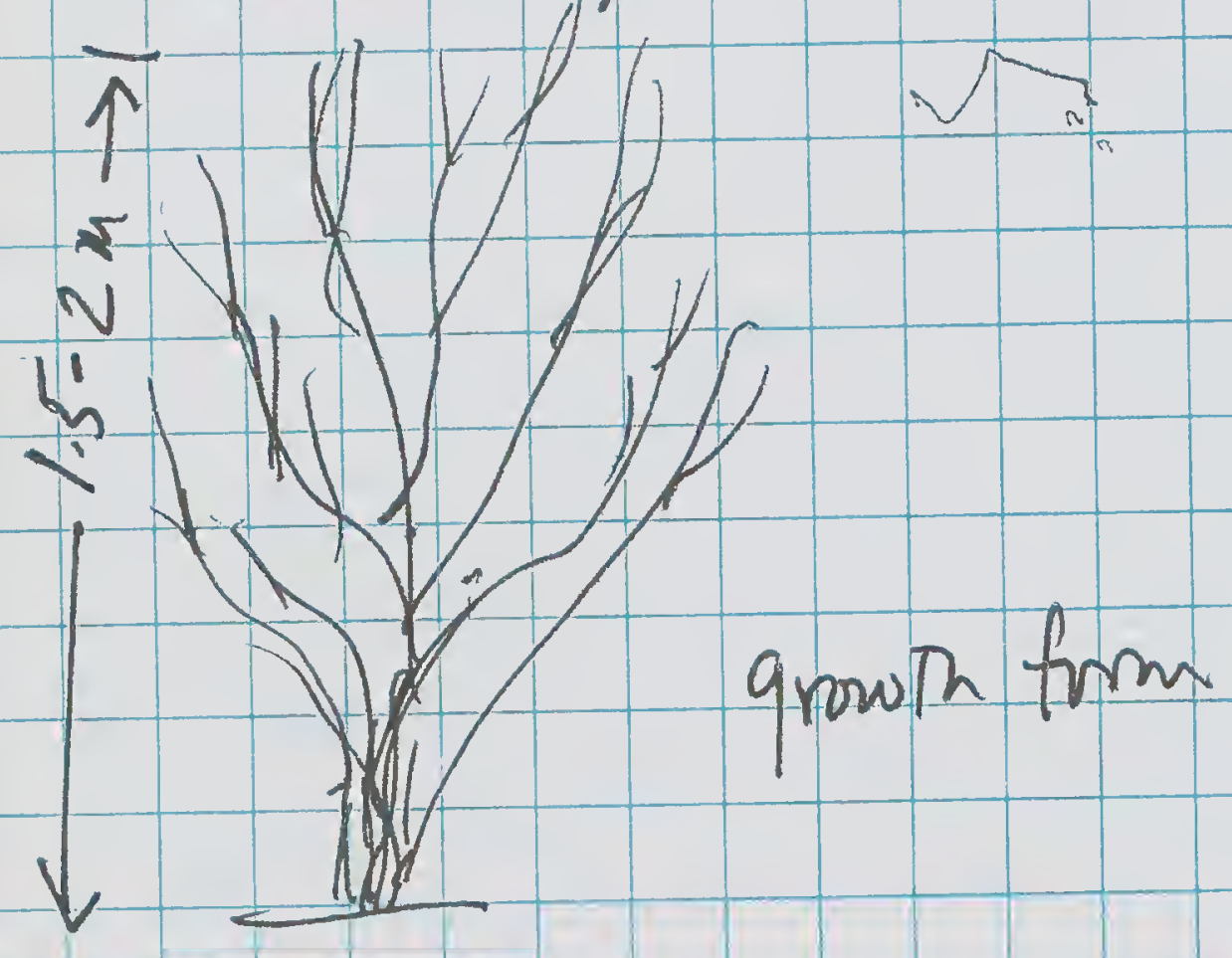

Curny Bush: Lysenima ciliatum

cool, clem, brisk breeze start 10:05

21 July bird trawsents in Durakoppin east reseme. start at Plact quadrat site 9 walk $X$. Turovgl Wongan, Then W. to main rd i s. alar r' to entrauce Trach, and baek E. tocar $50 \mathrm{~cm}$ sequents, $25 \mathrm{~m}$ each side

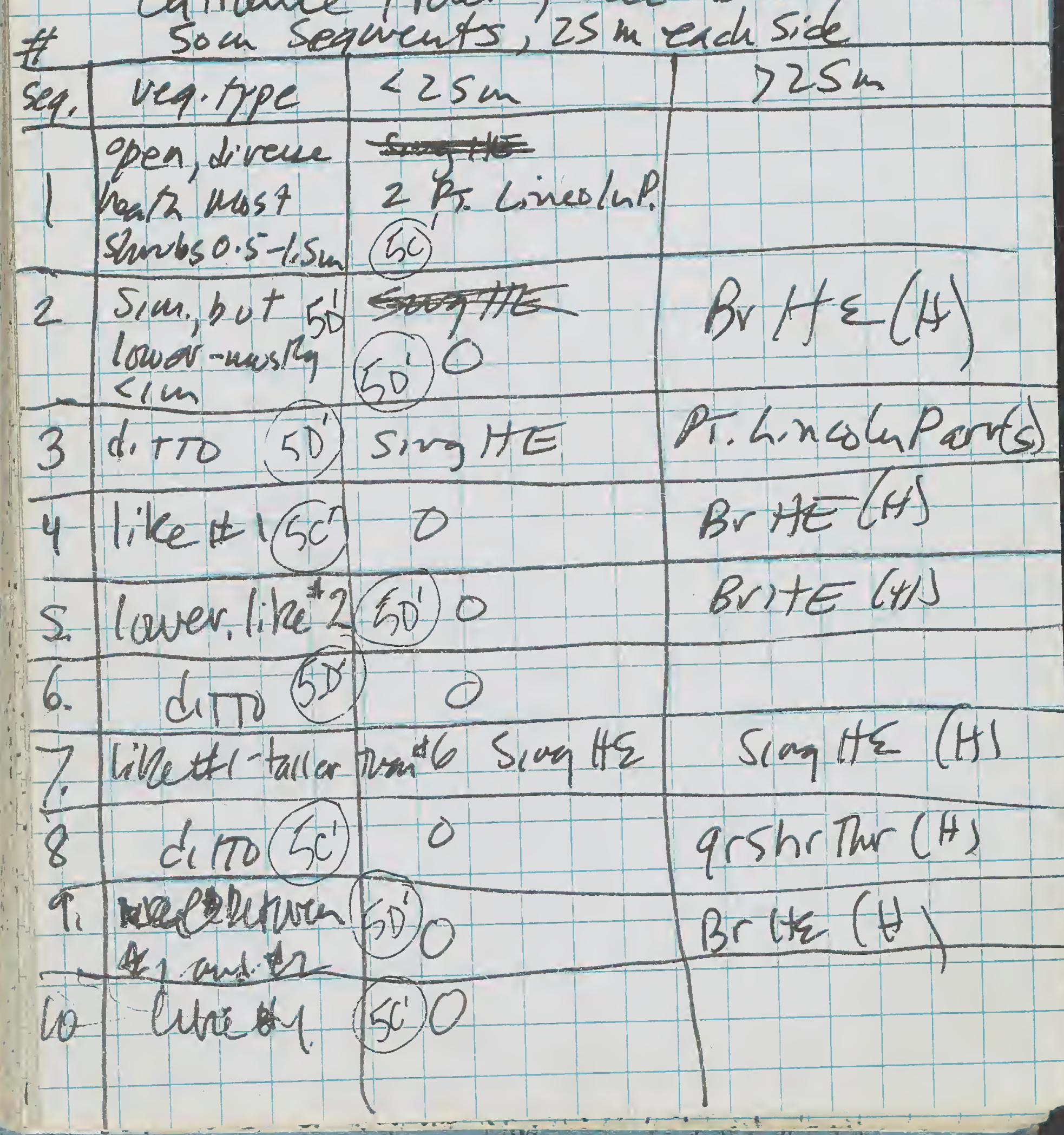




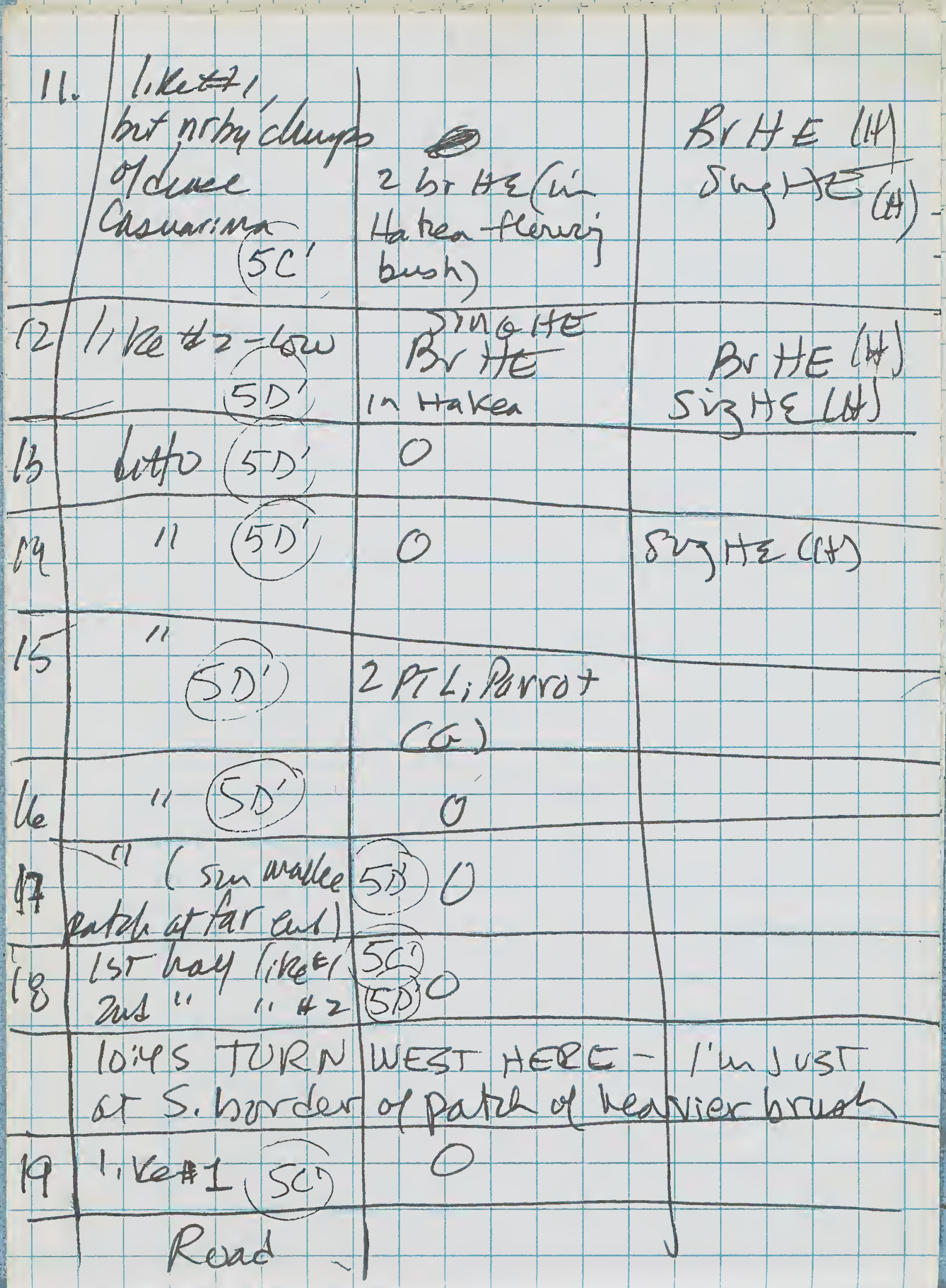

Idid one fune 'interior' sequent is mole aur even 201

Eysenima ciliatum "cupry flower"

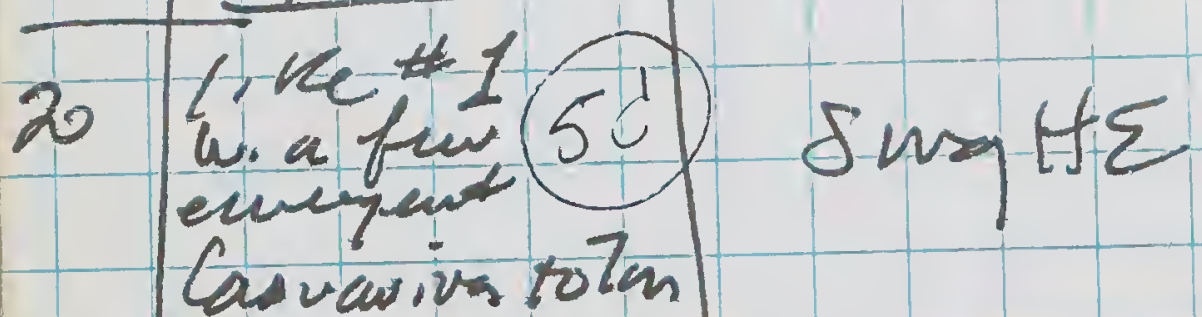

Reabiden proge SouTupand

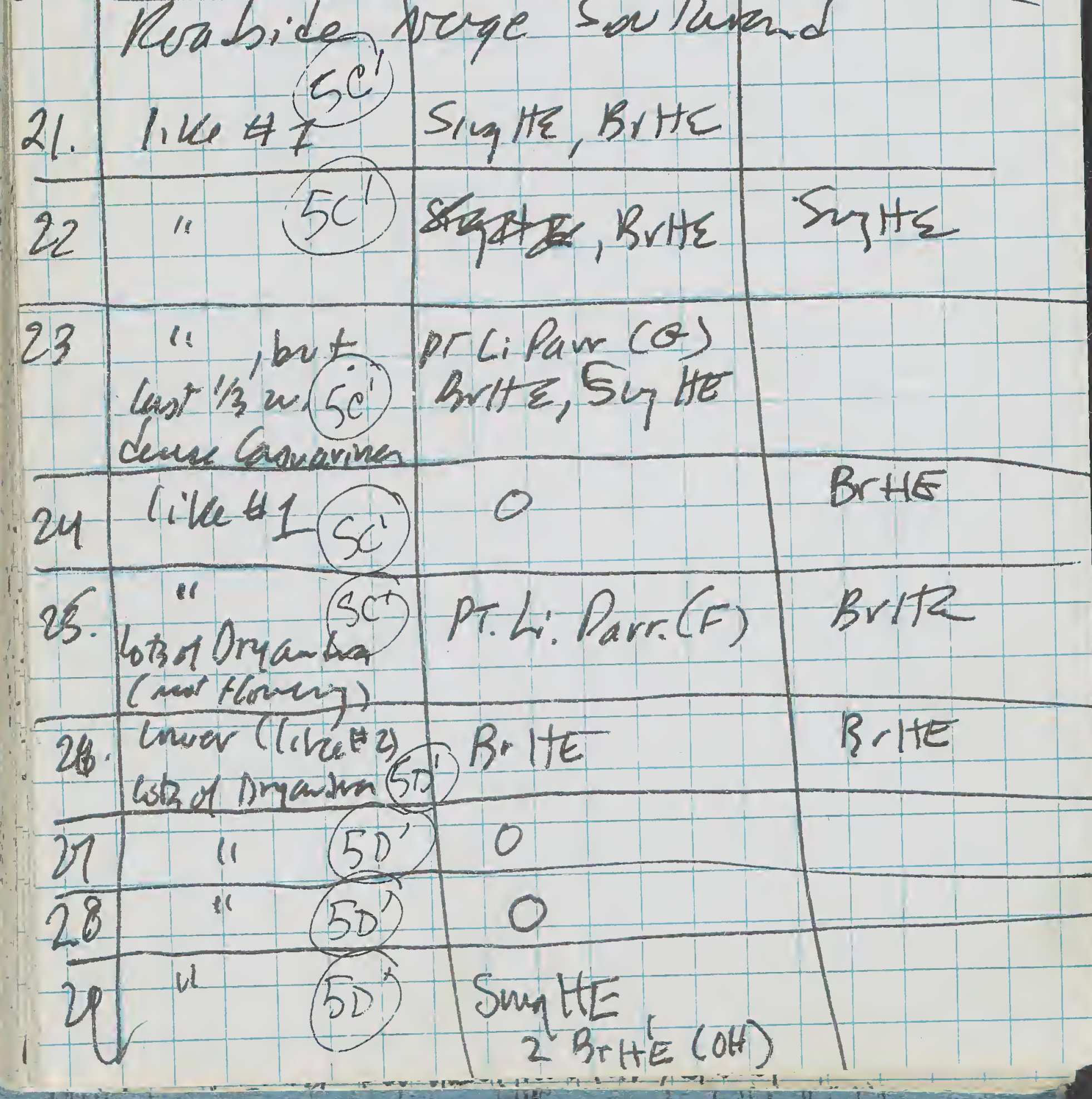




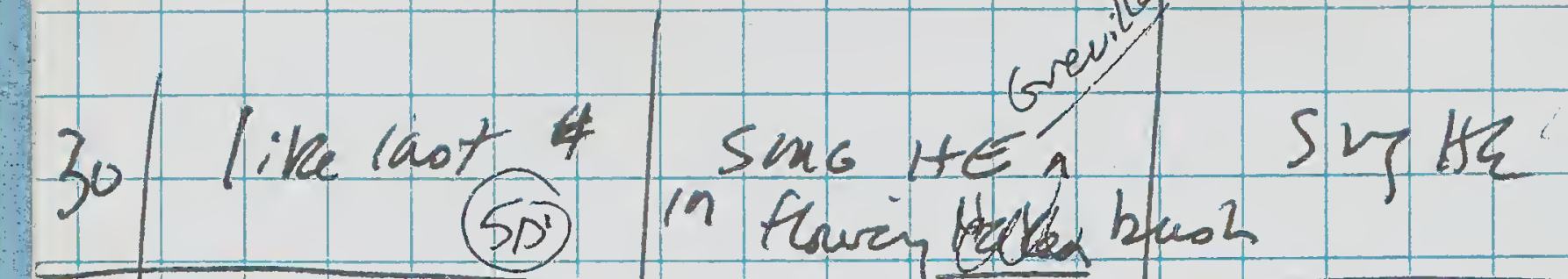

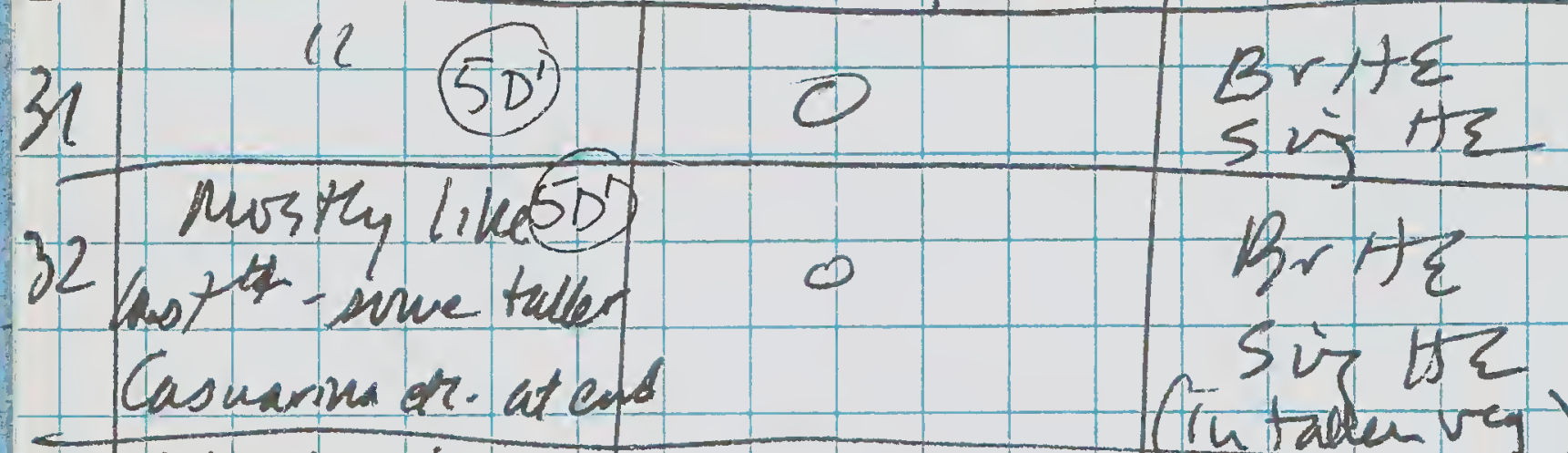

new 21/2m (5C) 3-4 BrHE Brite

- Cas woina Thents

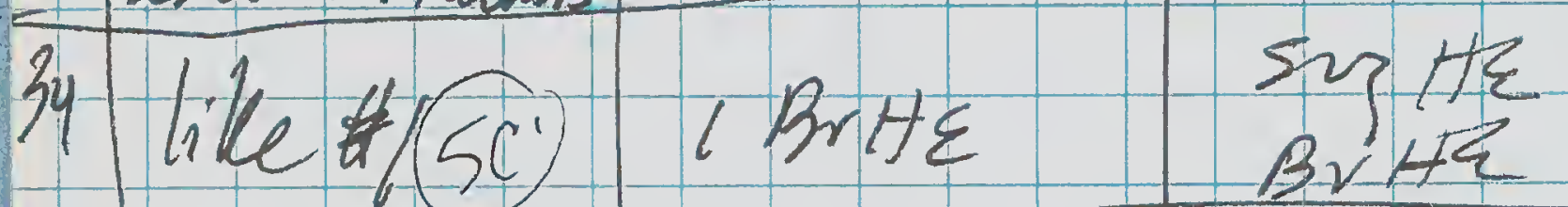

- BVAlE

- Vine 41 (50)

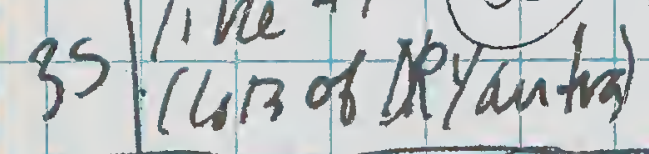

36 line 41501$)$ LSuyte

37 liket $15 i$

tar Dryantia

38 like \#! but 5C

w. mve dever

O

$0 \quad$ BrttE

entrare road

3e lou sedqe-bush

3e. Clemps (Liw) w $5 D$

nou of las varin alid? 0

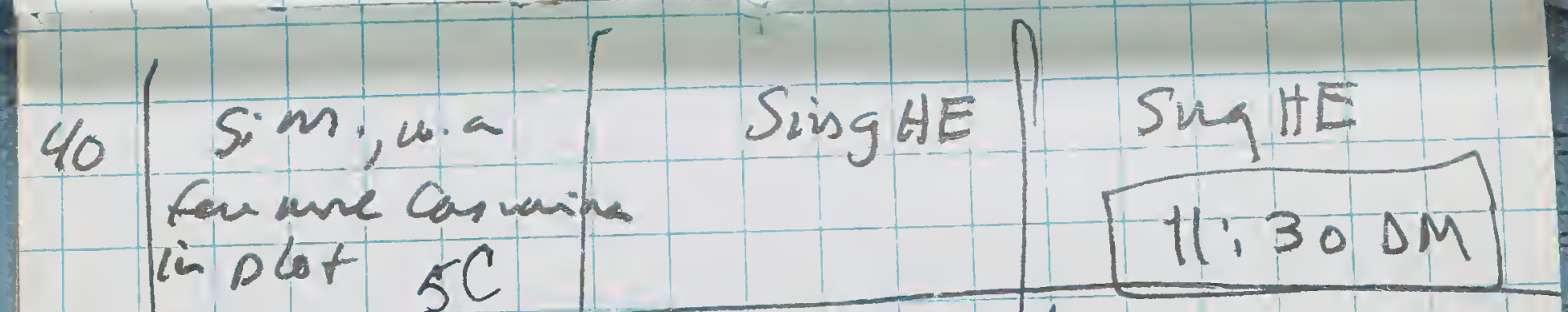

fou une Cosmaiph
in Dlot $5 \mathrm{C}$

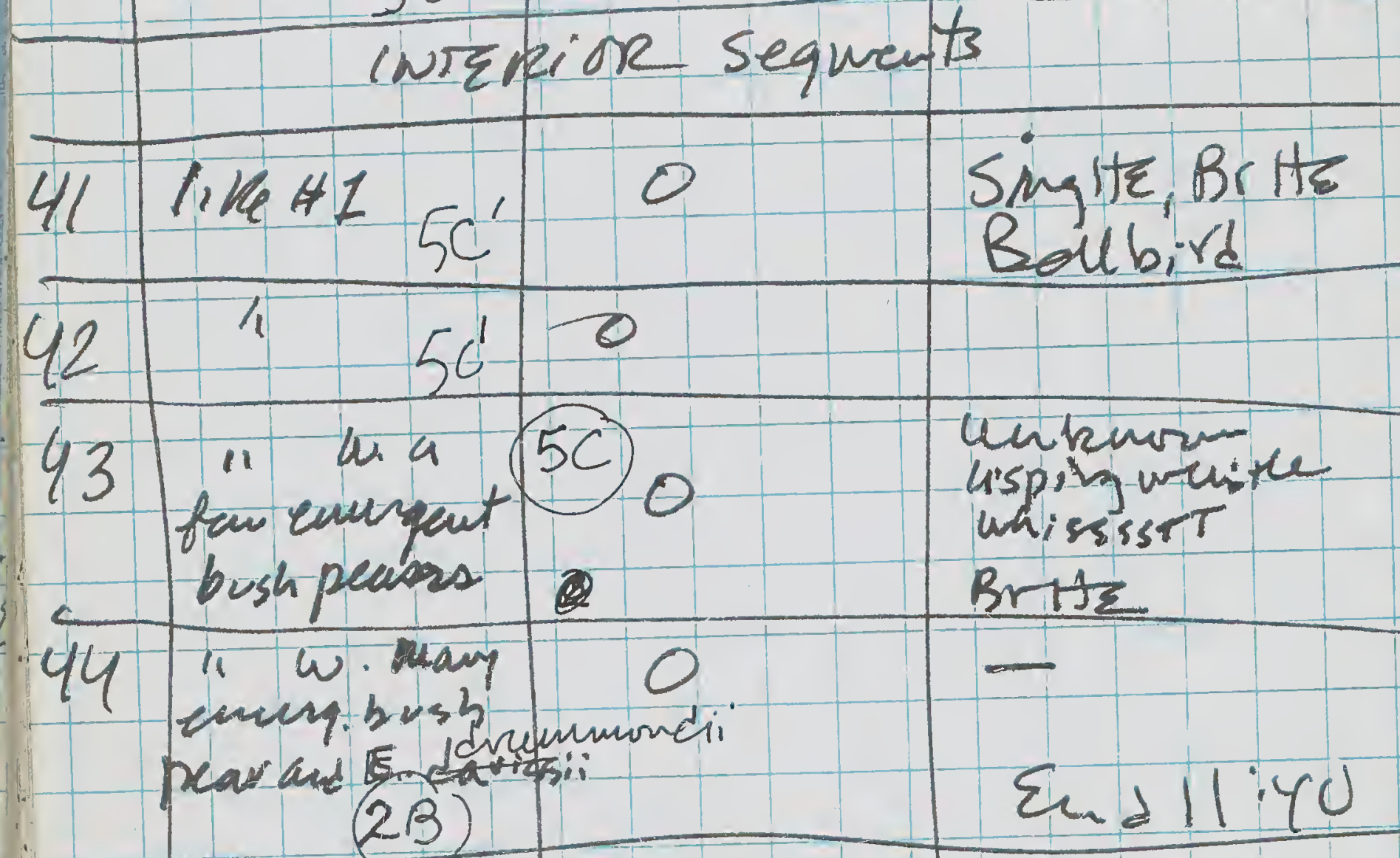

mileage at parkiz spot :988.05

at dot cu main roas: 88.3 
2) Swey 88 'Weeds' pes

sig. Habitat Paddock, Start at cross track,

$525 \mathrm{u}$ EDge Transeet work baek roward NE

11 st $2 / 3$ open w

$1-1.5 \mathrm{~m}$ buskes je

lout $1 / 3$ tall (to Sun)

Cosuariua pateles

2

- pen arasty $30^{\circ}$

suramiah lite

$\mu$. Scafered Wettle

Sandalurod,

3 Seusev, pot tremes mis Gr. Fautaid

clump (3B)

4 treent 7 3-9m W. wagtal

S. like 44 , but suctuos tell glems up to $10 \mathrm{~m}$ (B)

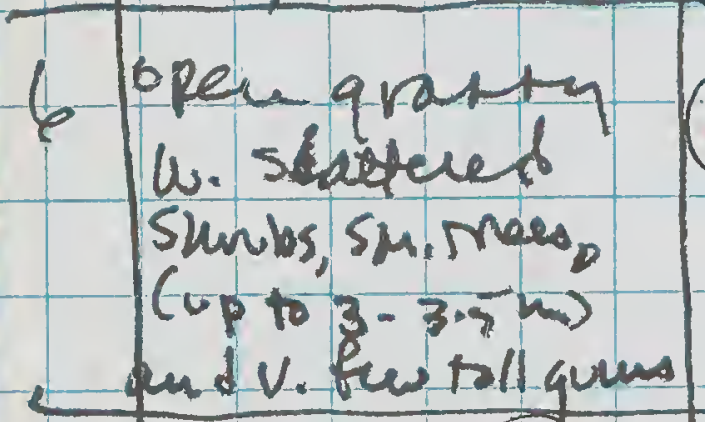
Sugtr

Th-bill sp

a shrthr

$2 \pi$ (16

8 (1C)

Shrubs 1 saplings Chat

9 Move deup - sume

nmall copses (B)

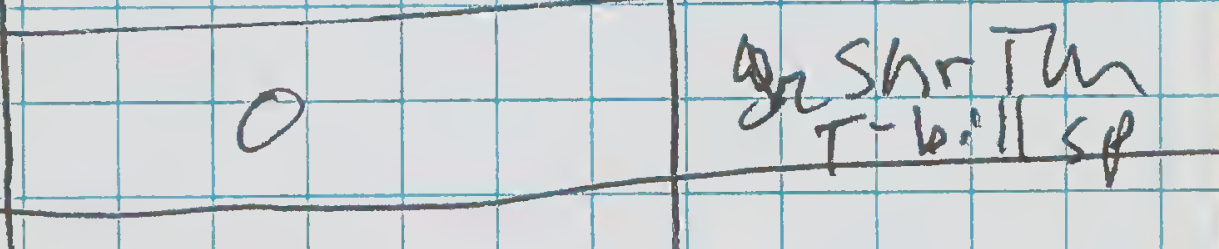

10 V. Low open

grassy wentual (IC)

cow surnbs aud

a lewvitall (20m)

Shlumen quens

II opensoulya.

gras deup, $2 C^{\prime}$

puttud low sturlas

sonve watte papling

12 Hetrioqueverear shrub/sapling/

grass CHT 3-3.5m

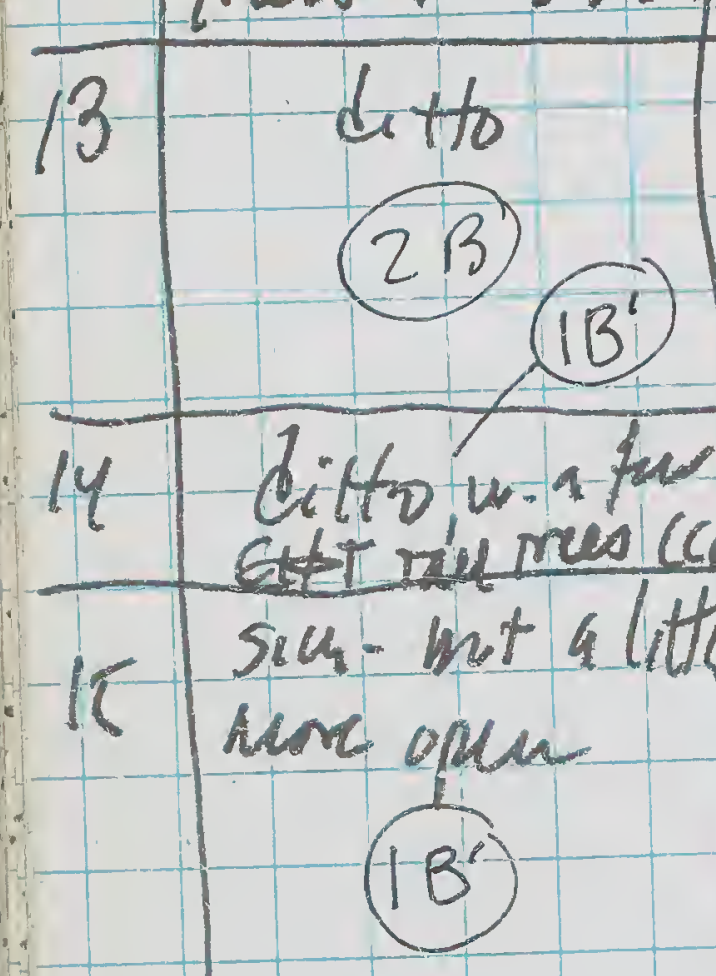

\section{(2B)}

Thounbil sp

Sincte

qRef Whitler Gr Funtaid Simate Bromite Thitlsp

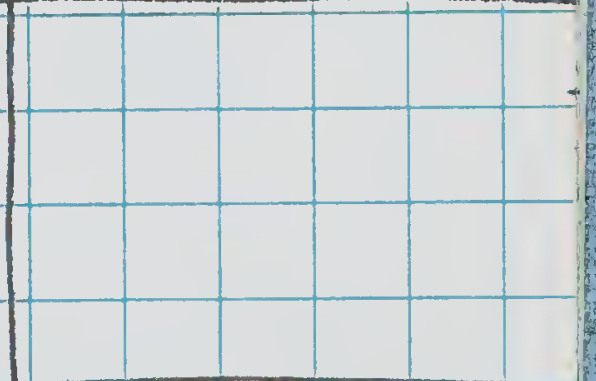




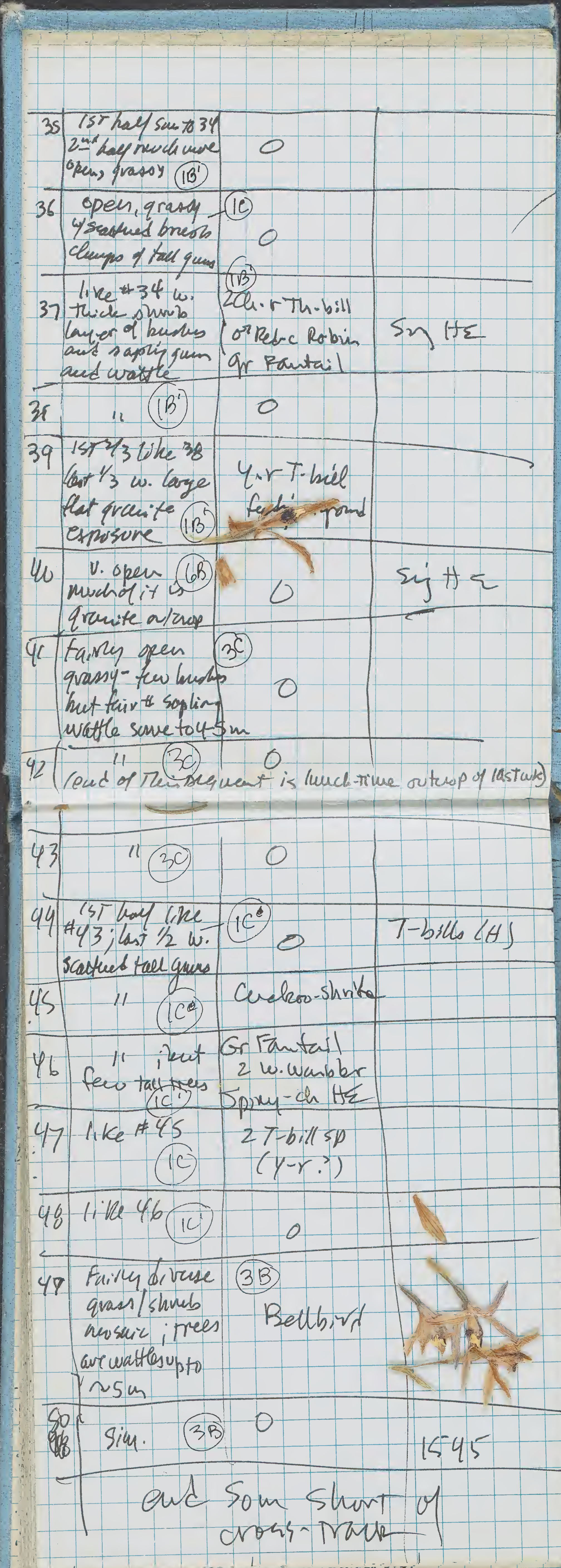


22 July. Durokoppin East - É. Side aloma Mission Rd. Trauscits of Som Sequn $15 \times 50 \mathrm{~m}$ wide; read sidetinterer. EDEE cool, Plc, s1. breege SDorT : :20 II $v E G \quad<25 \mathrm{~m} \quad>25 \mathrm{u}$

1 Gr Fantail Grshrthr opeubrush w. 2 Tree Marta (OH) Bellb

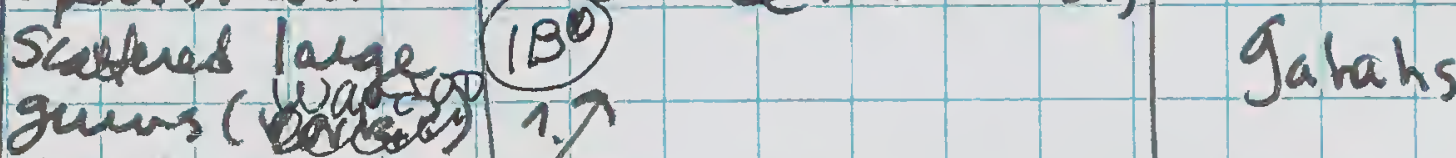
To $215 \mathrm{~m}$

smu, but a. elegant parrot

2 deven bush (J) Jree Martin (ot) equer bigtres (IA)

3 dito, but

3 asuacuina v. delese

(1A) 0 Singhe
A)

4 dito (A) $2 y-r$ Thambil sovita trees (quirs)

zeleqaut parrot

Pately sunibs SUM HE overstumstil $B$

rastent ly guens (B)

6 Tut $1 / 2$ V.open, 2 Galuhs (oH)

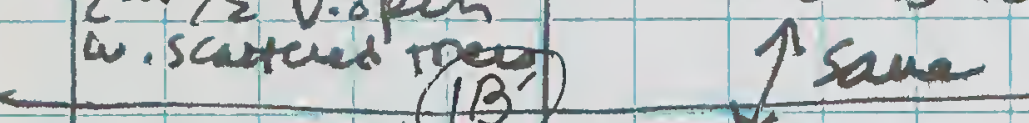
Bellbrrs live lost lof 2 gatale (revelud) of HC (B) Tree Meutinfo A) (i)

of $1 / s$ hay dito 2 galahs $(p)$

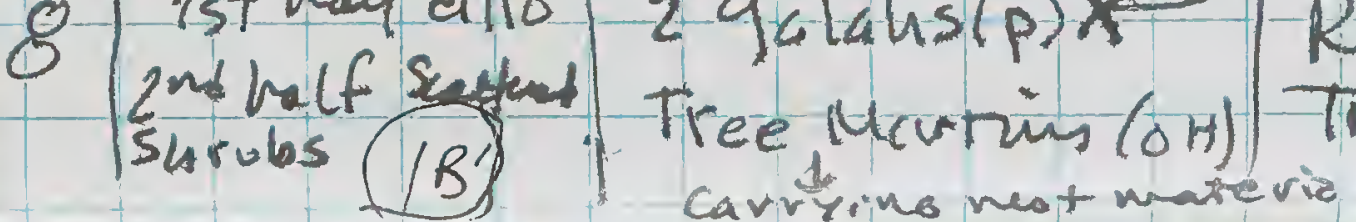
Raven (paddode) Orshr Thr

9 V.oreu ywaided \& Dowestic 2 Seluovoum - (1C) Pigem (P) wuch bare qrond galahs (P)

10 deto $1 C^{C}$

\section{1} 1 "(C") Ser. Gulaks (p)

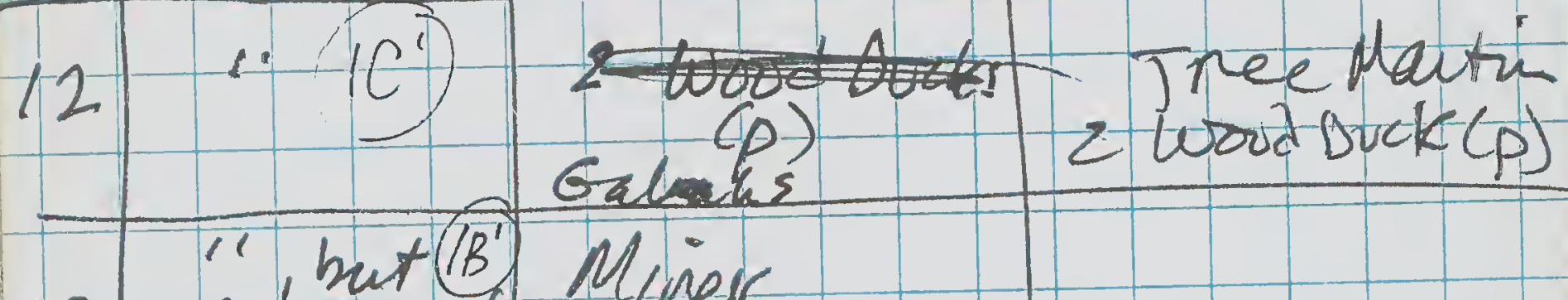
13 modenato demia of $1.2 \mathrm{~m} k \mathrm{zh}$ shiviss

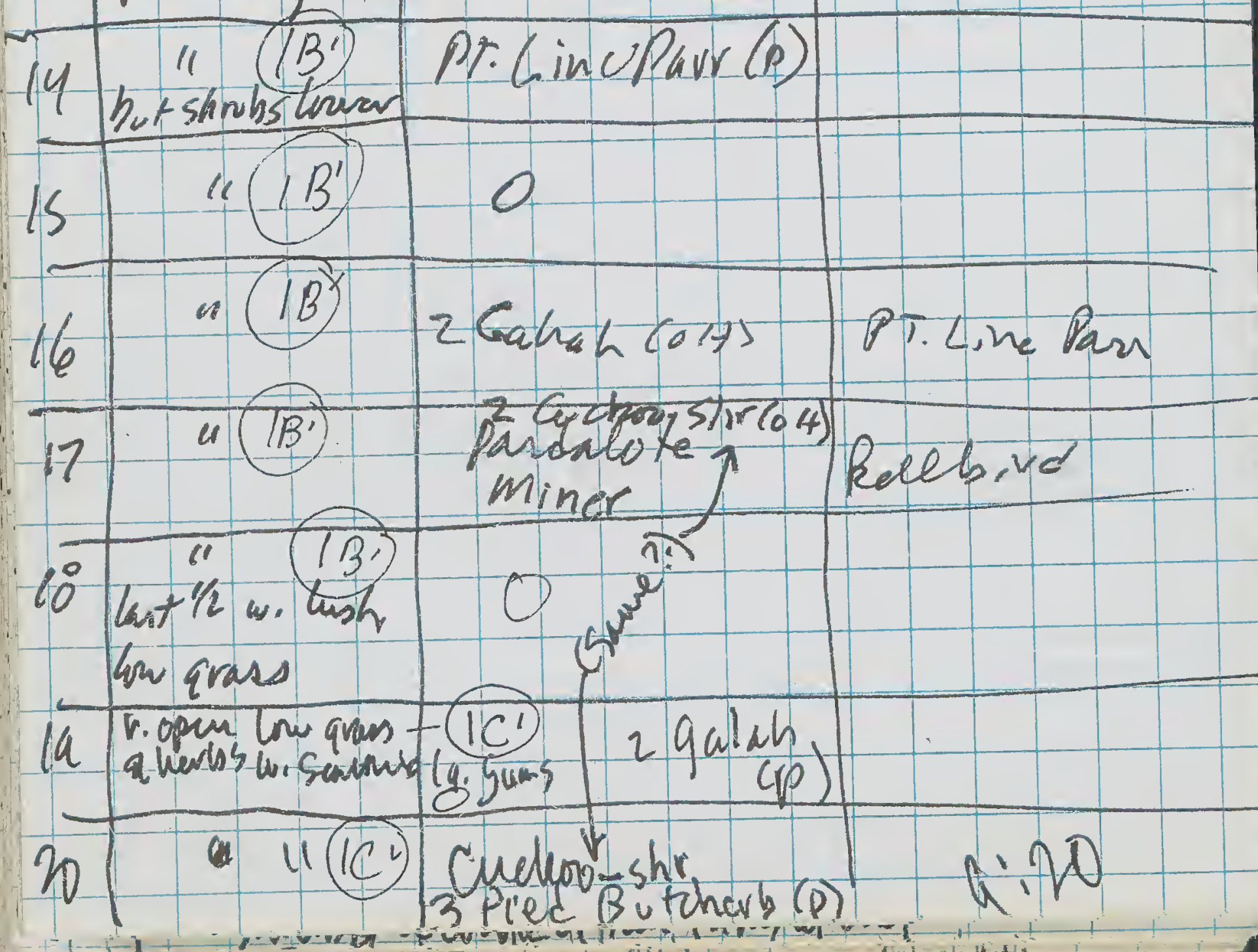


D. $\left(p_{i p}{ }^{\prime}\right)$

oun mostof

Pilipa Aarvey, nexpert to Mark Harvey Durokoppin Mon swotch to Interior trancat

Toel gunsw. Purdalote to the Ui, estusa 4 gahah (p) Tea tree Thichota

Heterog, open (1B) Pardalote $*$ (P)

2 /briesh/bare/ graso a.ten Wando

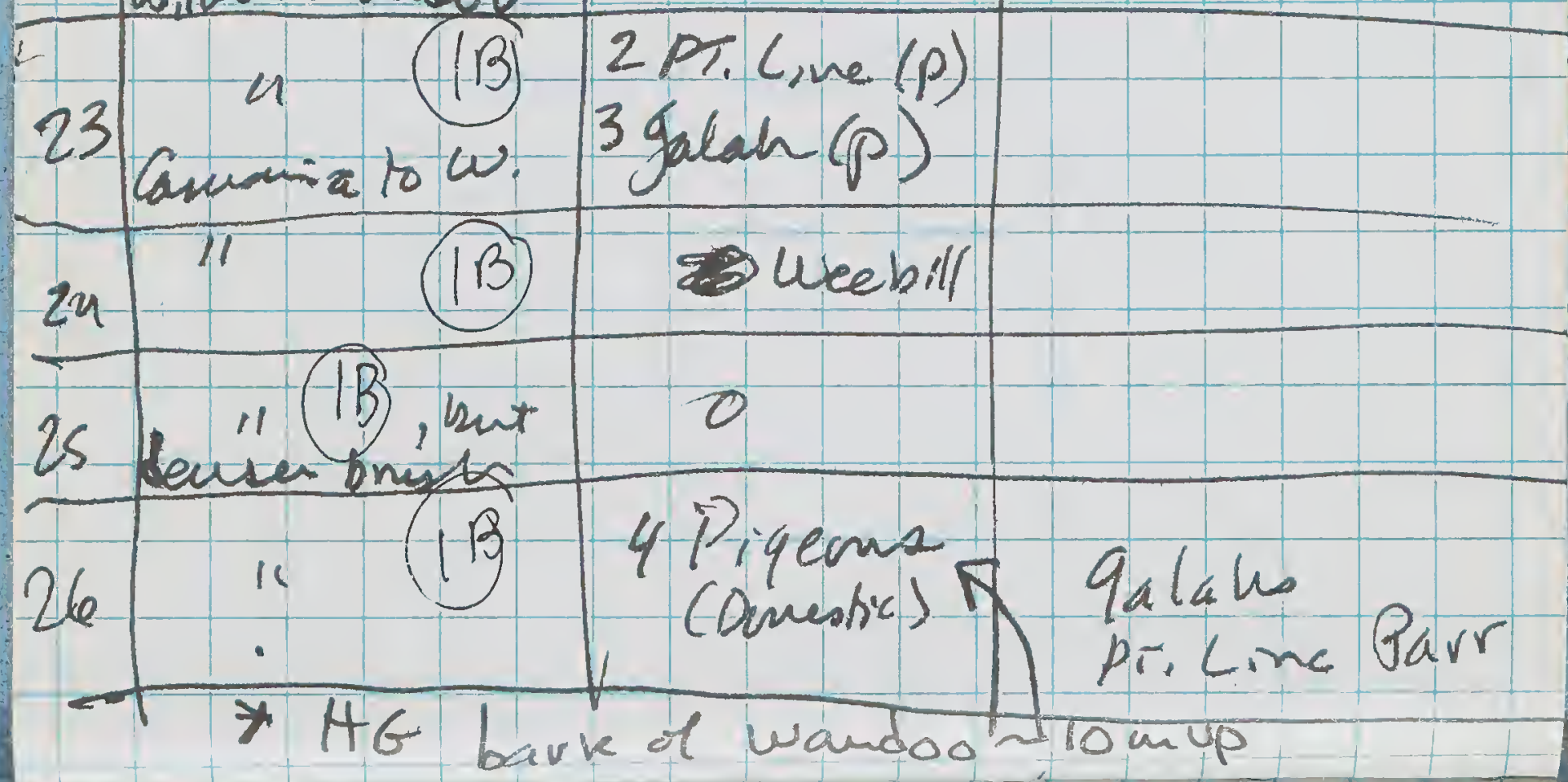

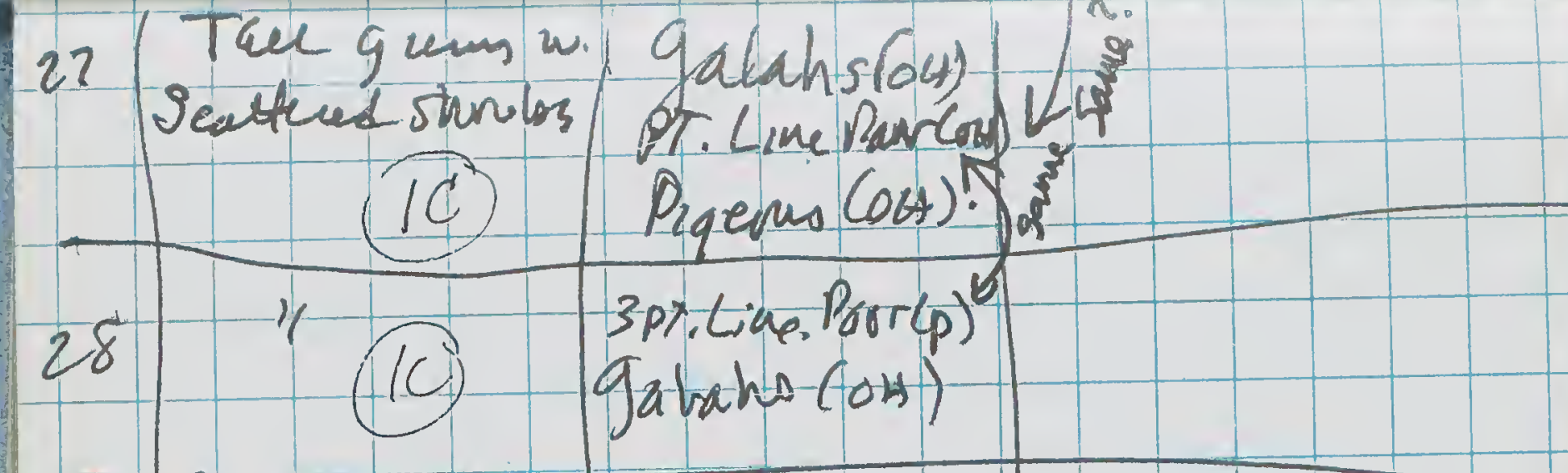

Sun, but sonu $1 B$

29 raller cluaps of (13) 0 Casumiva

30 Taee Salum 9 (10) Wavdoo open bou quass w. scutkend hushos

\section{Jalahs}

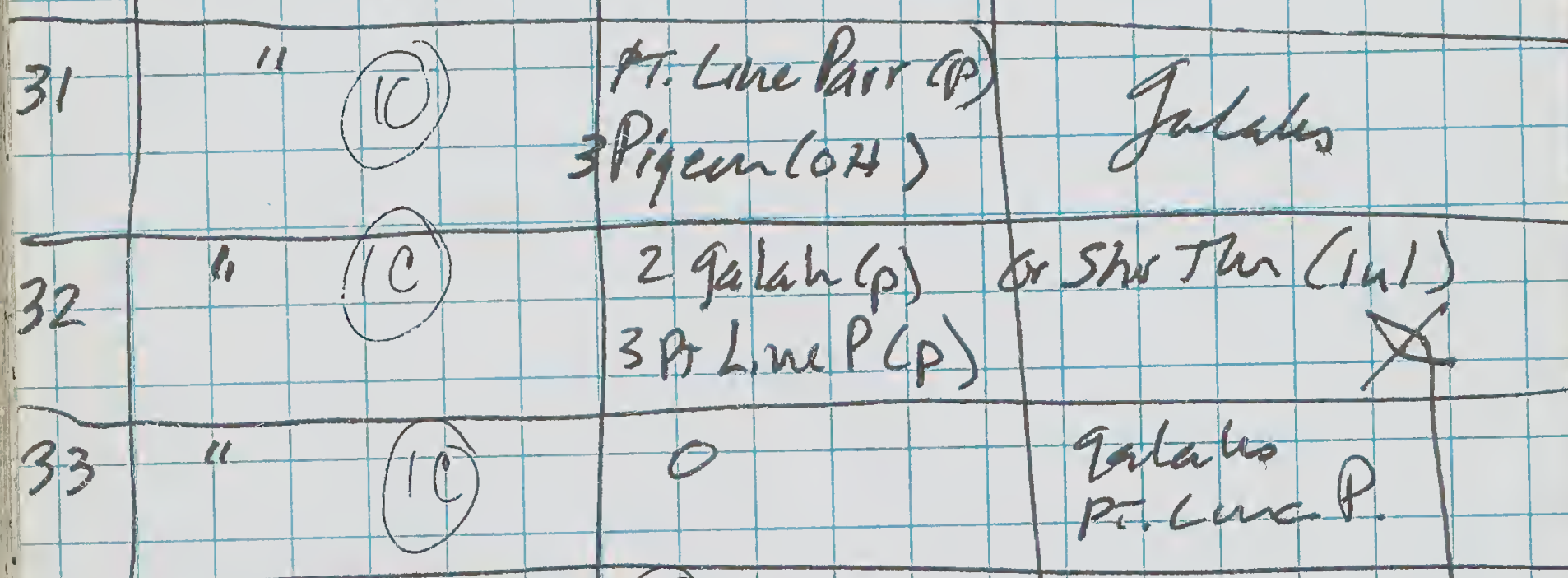

sim, butsunbs $1 B$

34 densier, max heterog. 2 galah $(01 x)$

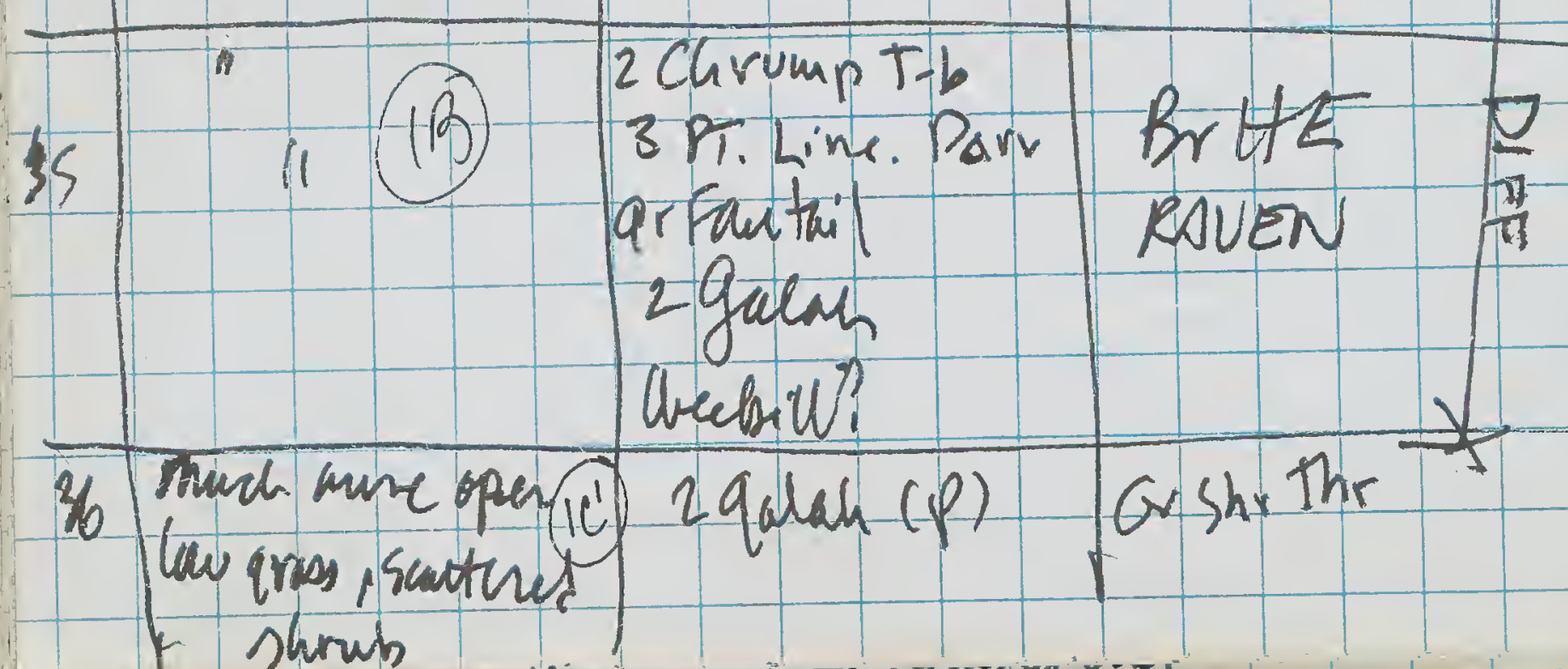


53 deesengum und decsen gum und/C scotenad shrub

54 11 10

2 cuck-shrlp

2 PT, Linc ( $P$ )

55 Vopeng wom wosdl. (IC)

\&e Lachin shrubs-low heabs, ape o

\begin{tabular}{|c|c|c|c|}
\hline & 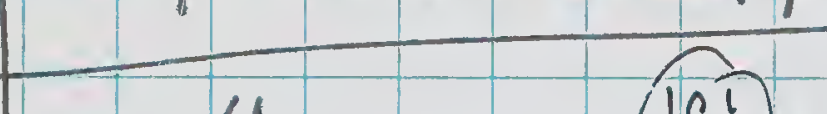 & & Pr. LucP. \\
\hline & $\left(1 C^{\prime}\right.$ & 0 & \\
\hline & $u$ & P. Linc $P_{1}$ & \\
\hline & r & & \\
\hline & $u$ & PrLuc P & \\
\hline & & & \\
\hline & Soure low $(1-1.5$ & & \\
\hline & Shube & miver & \\
\hline & cut in & $\$$ me & $10: 55$ \\
\hline & wattel to $7 \mathrm{~m}$ tan & 0 & QrshrThr \\
\hline & Cosimive (2A) & 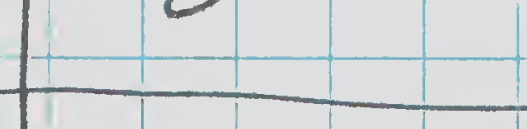 & \\
\hline & "1 but some tai & 0 & PT.Linc. P \\
\hline & J wons & & \\
\hline & 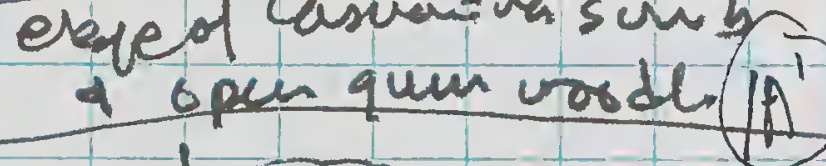 & $\begin{array}{l}\text { BKsh K,te Cou) } \\
\text { (Don't Count }\end{array}$ & $f($ Track $)$ \\
\hline & 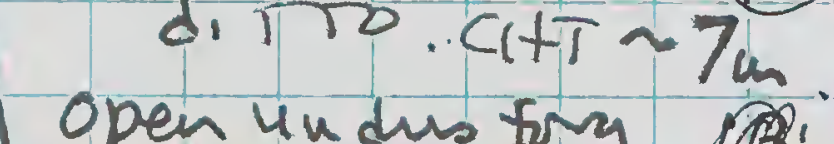 & weebillt & \\
\hline & open undus tory & & \\
\hline
\end{tabular}

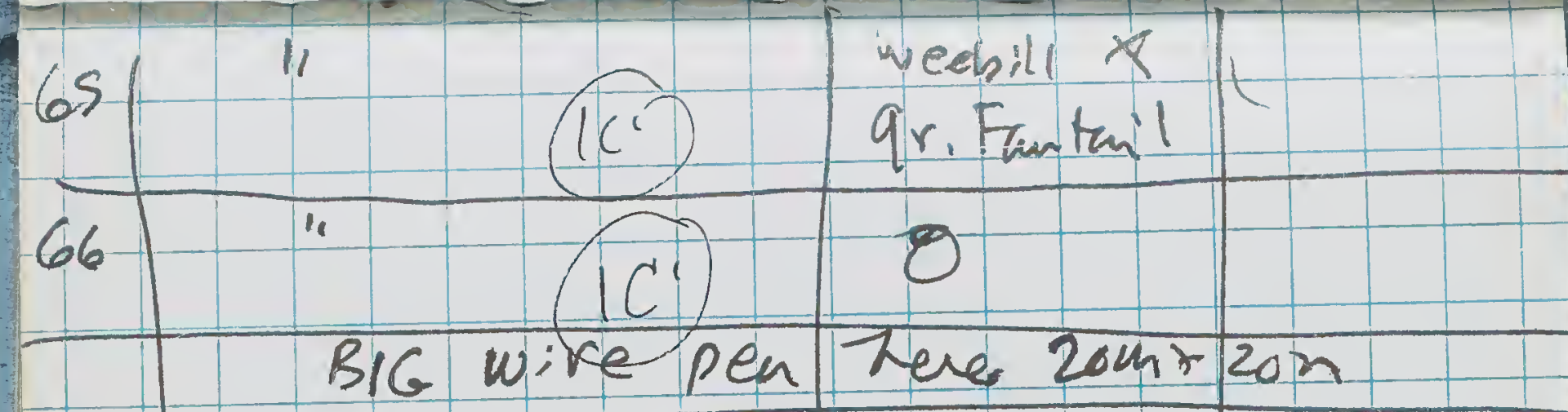

\begin{tabular}{ll|l|l|}
\hline 62 & " $\left(1 c^{i}\right)$ & 0 & \\
\hline
\end{tabular}

BIG Circulat track hen Tall quans (to zom) $283 . \ln P(A)$ but V.open undustiry

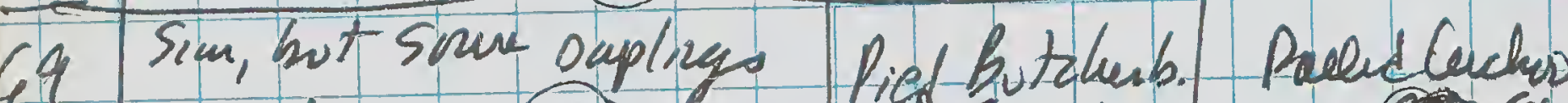
ols (1B) Esub.) (A)

70. lower qum woold w. (Cat T/ 5-6m) Ballbird

70. York a Waudoo-sprenti (IC) frim-openundustory P.Luc. Parr

\begin{tabular}{ll|l}
\hline 7. & T.bils (sp) Grshrthr \\
gatalls
\end{tabular}

\begin{tabular}{cccccc|c}
\hline 72 & 11 & (1) & 0 & $9 a$ cins \\
\hline 73 & 11 & (10) & 0 &
\end{tabular}

44 "butar edge of - (c)

44 duse casnariva somb

10 6

15 (10)

13

16 Mozply wattle (to $5 \mathrm{~m}$ ) 30 ) oply undestory 
$27\left(\begin{array}{l}\text { sim, but quento } \\ \text { wiwattle }\end{array}\right.$

Gr Shrthr

Sutte

$P_{T}$. Cine $P$ 28 Heterg. Shrobu.tare

4 Godah (or) Weabill

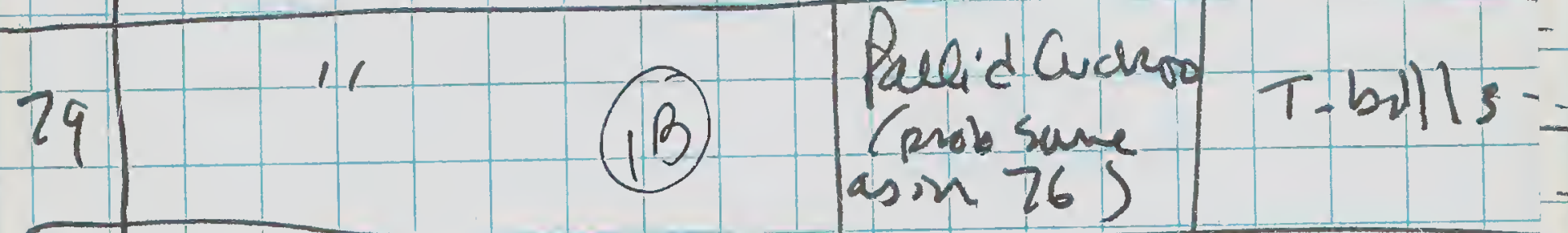

80

(B)

$P T \cdot$ LincP galahs

lauded 150 in taur

lan uiddla of staked ptit]

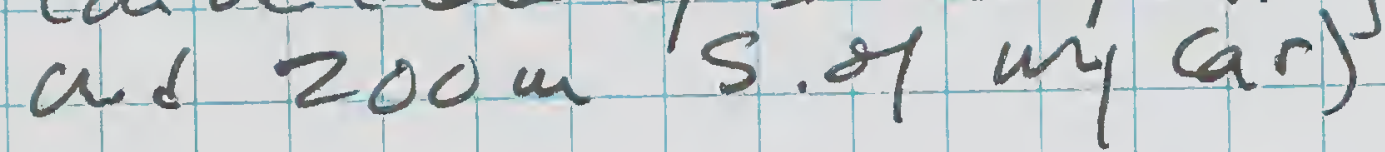

Car is parked $1.3 \mathrm{~km} 5$. of Ja Brow rd $x$ Mession

$4.3 \mathrm{ren}$ from Thes courer to CSIRO love - verge is veretated all thu way

22 July. Corridor Surrey. W. Side Missian rd workin S. from 5. Conven 'Weeds'

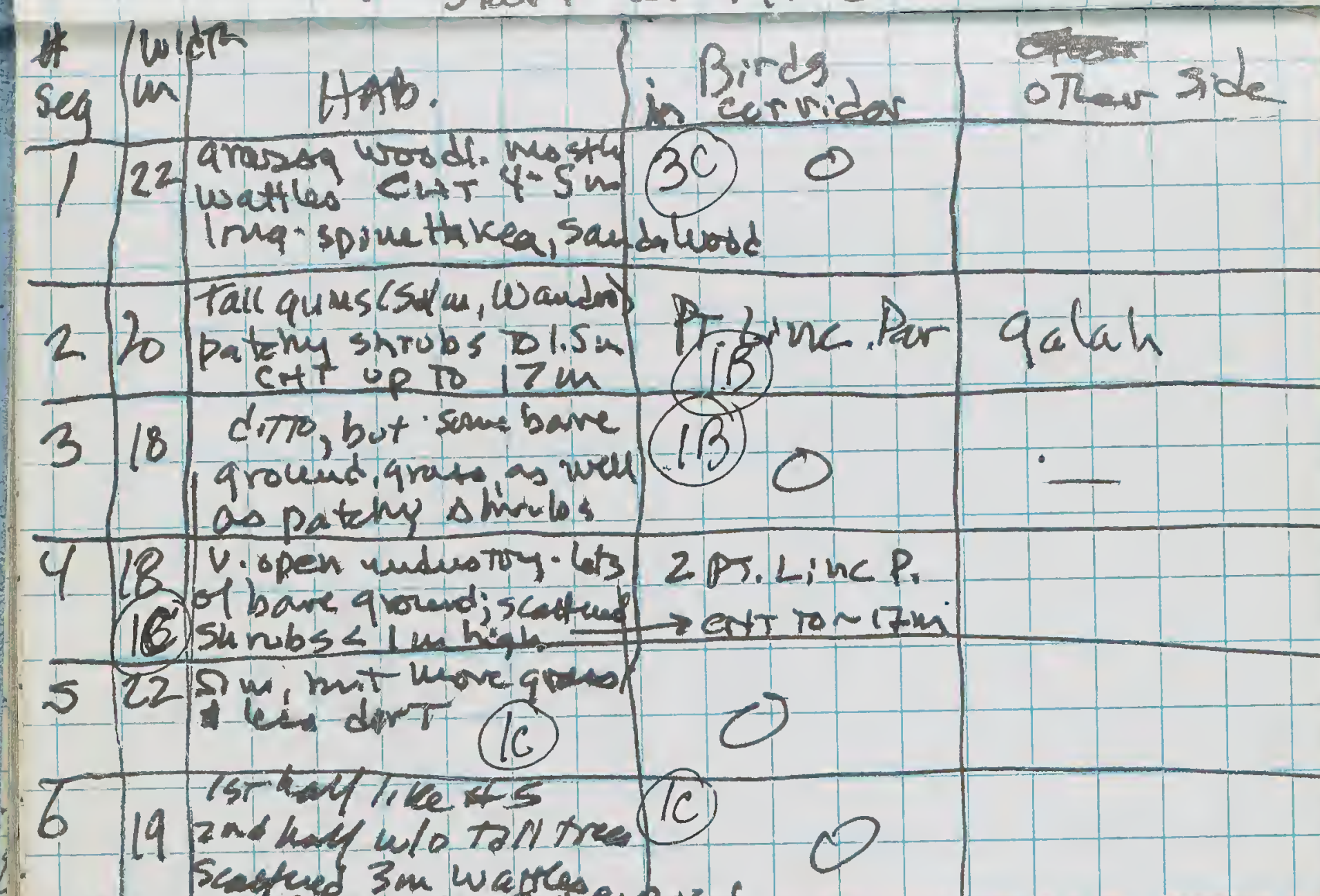

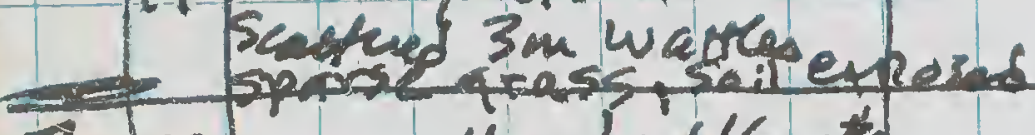

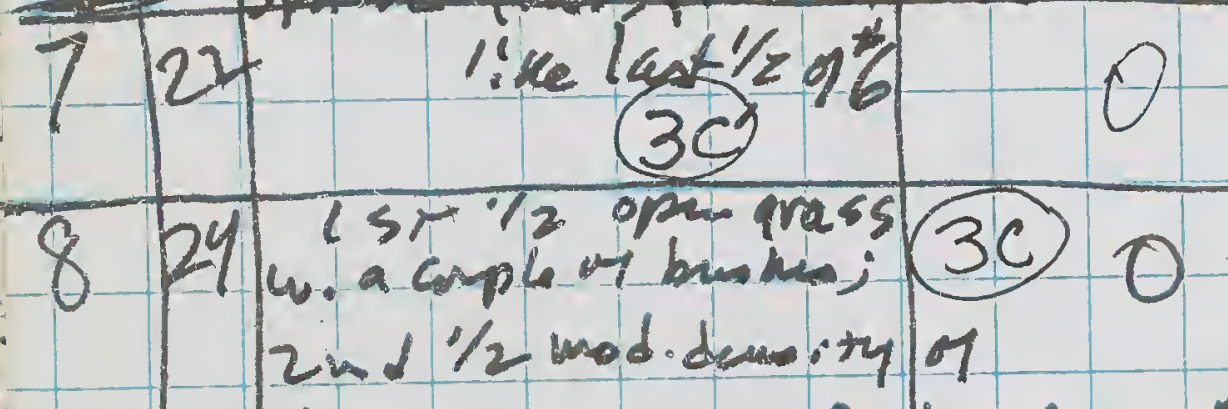

Wartle to $2.5 \mathrm{~m}$ but otill mosth orem 9 r

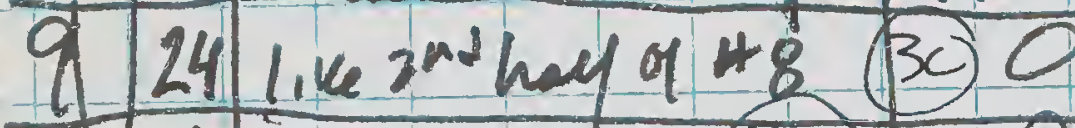

to 19 11 (30) 0

II- 18 ópen grass; v. Seno $6 B$

scatked Sm. Imoluse

1220 V.opengrassy; mod. (6B) - wathe d l taken las $10 \mathrm{~m}$

1319 open shrublarass w. $5 c^{3}$ )

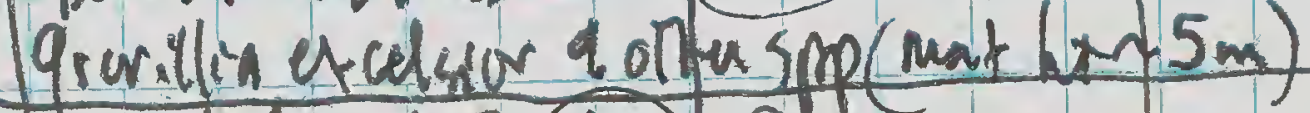

$14 \sqrt{18} 1 / 5+1 / 3$ (1.ke 13 (613) riest 24s v. opeu qrats

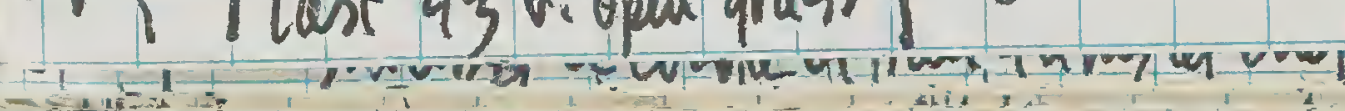




$$
\text { Thue 3:40 Pm }
$$

at This pt. 1 an $\sim 350 \mathrm{~m} N$. of entrace To G. Formurase on É. Side of $\mathrm{rd}$. I tersssed rd. and stouted back $N$, cuscusing. Verge

\begin{tabular}{|c|c|c|c|}
\hline wingh & n 1 & sping inge ? & OThers \\
\hline & 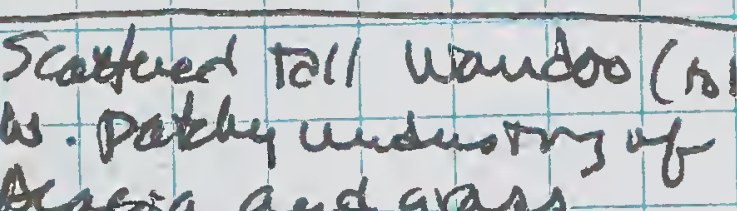 & 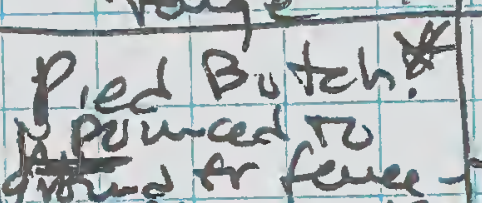 & \\
\hline
\end{tabular}
Q2 21 (Bi) 11 fer 1 st $35 n$ TF Miverx DiFF

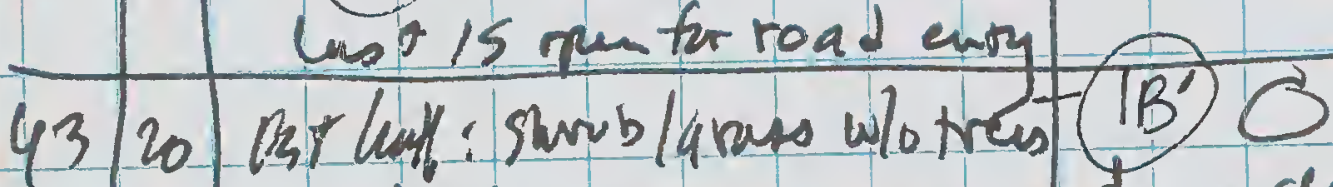

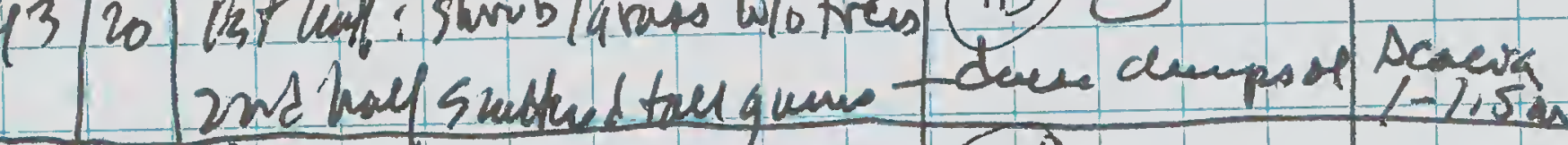

4422 bu breshdeuzp w.open $1 B$

- around - a couple of tan quans

QS 22 Gen" orchand "Type Good! of supling watte, queuv. little modentry. lots of dirt CIIT 3-4m

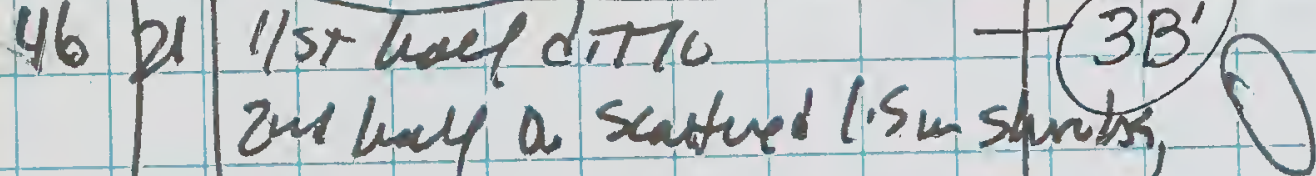
fever trees

4720 low trees scatcied w. 1B.

4720 clumps o1 duse 2-2.5m bipesh $1 \mathrm{lg} . \mathrm{gman}(\sim 18 \mathrm{~m})$ 2nd hall mave open $3 B$ 3B5 twiver un padduch

49 Ro he ferca. - low gouss watteg y Miner $\rightarrow$ Flew to ground $C H+23 m-2 B$

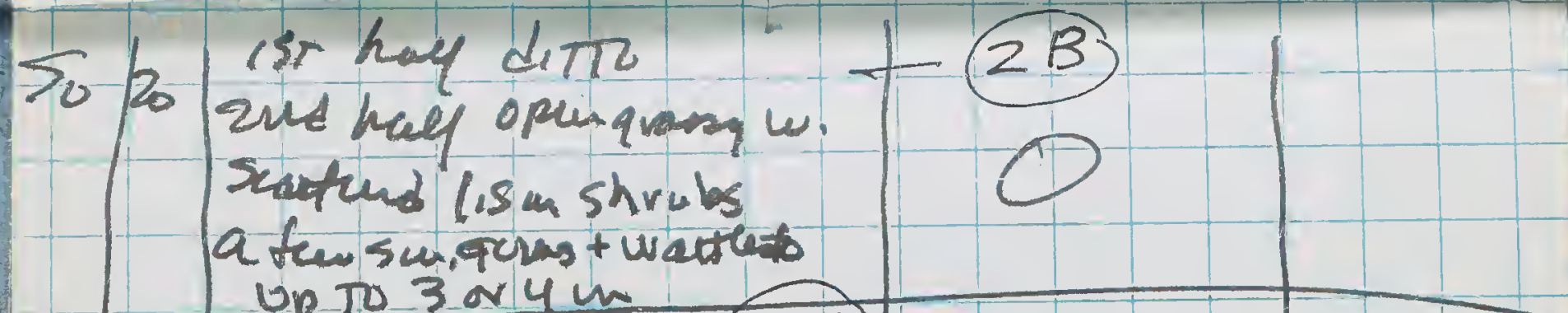

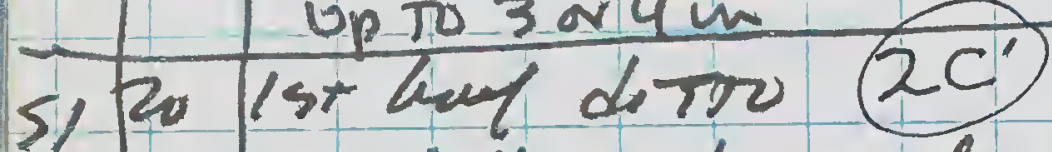

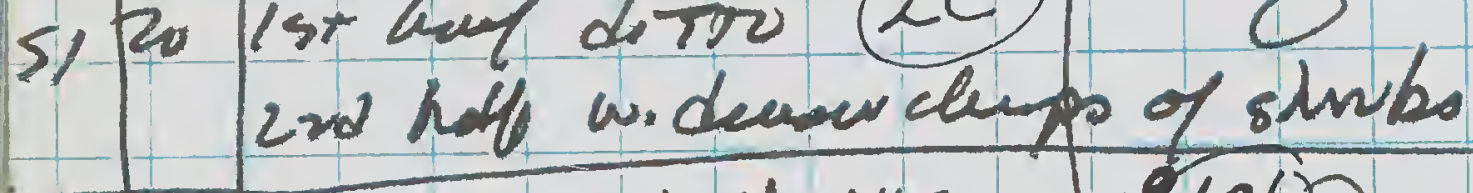

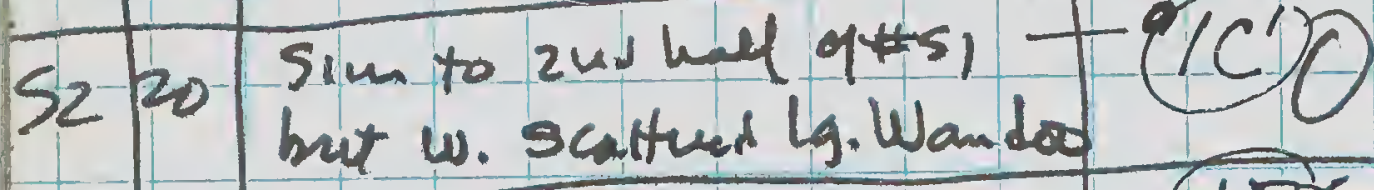

3 po opeu wandoo wordl. $\left(B^{3}\right.$ fatchy undustry of qrass, 3 Yif Miner brash chumps to $2 \mathrm{~m}$

$54 b_{0}$ open liandoo w, v sperse low underst; nucla bove dirt

So 29 (st 13 likett 54; last $2 / 3$ + deme alunbs lis-2:04 No rees (IB)

Mit of dunse shrub patdep and $\sim 1 / 3$ open graps Nola. frees

57 Sim, but Shrubs to $2.5 m$ (2B) 0 a a few sm. qums o waltes to 4 m 5824 tower prees, mave opuig 592011 (2B) (a) 22 kr hal u. duese Tichas of - (5A) Qo Gosuarm; and hall ope arass a. féw shmibs 


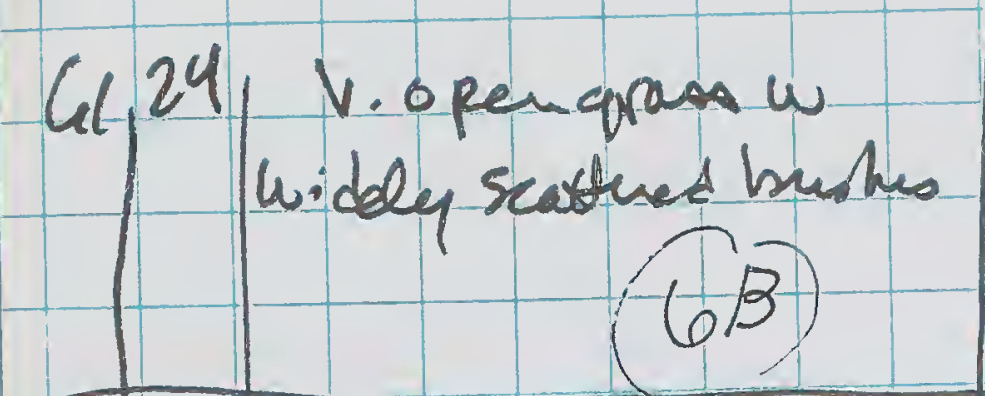

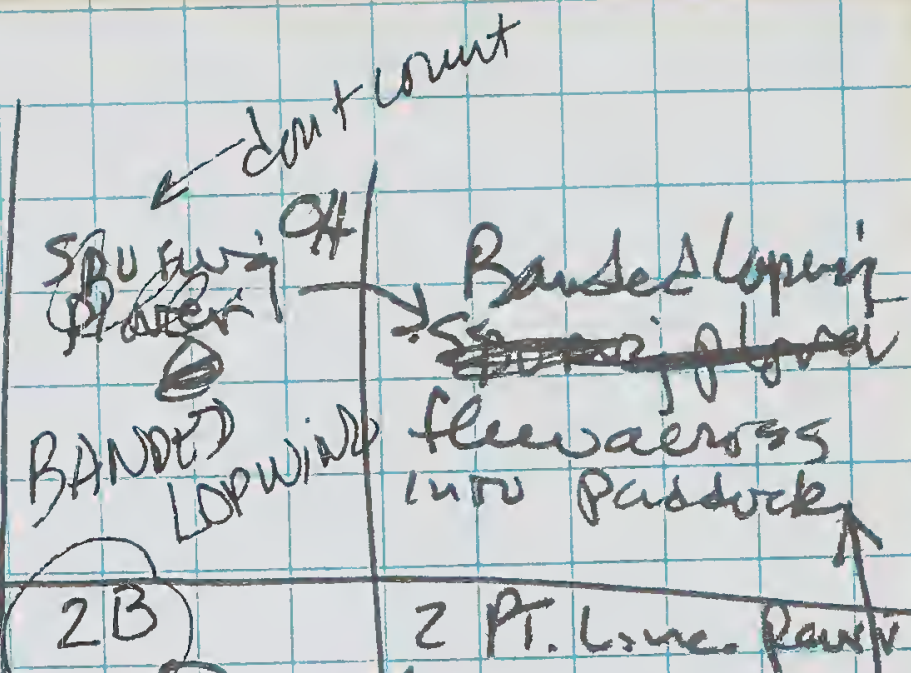
624 ist hay ditzo ${ }^{2 B} 0$ scrulb and sm. gquus, wattes to 2, $5 \mathrm{~m}$ feesin in passuger acsoss $r d$

6328 like 2xd bar of 62 (2B) ples sour quevilleato $3 \mathrm{~m}$

64 of open qrase w. widery proxtered demps of wathe, quevillen cos unim ro $-3 m$

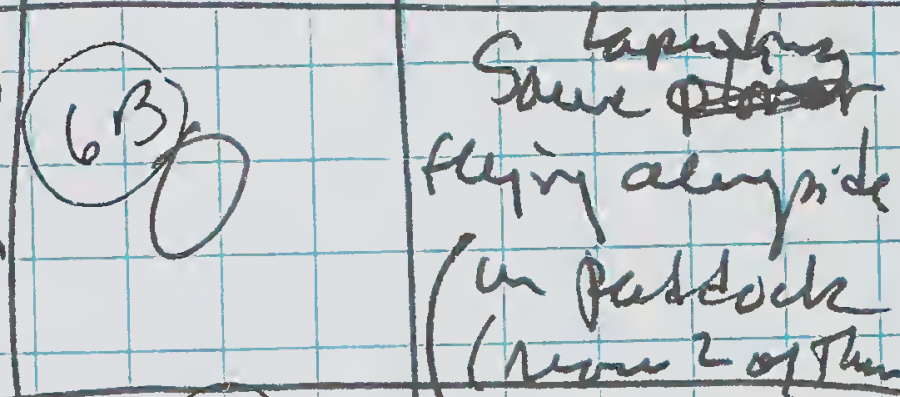

\section{2 opengrass 4 exotic caterdicasses ber}

(6) 21 ditto, but a fow 0 (6B) (nem 2017un)

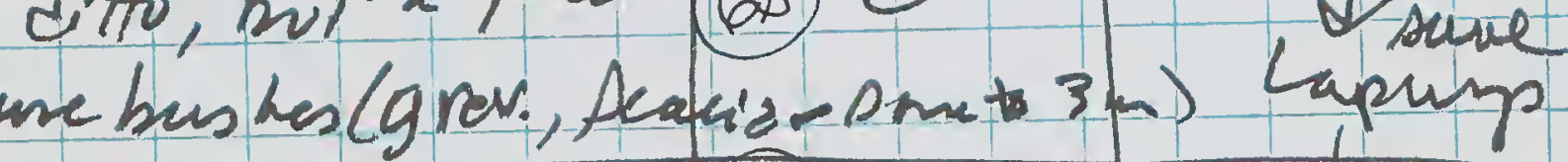
as iq betwe $15 \times(6)$ (6) 0 vave (5C) 11 openbuedirt 0 Casuaviun qraillen, danfia mestly < $2 \mathrm{~m}$, mut qrevillea to $2.5 \mathrm{~m}$ (8) ho open grass wi sextued Srug HE

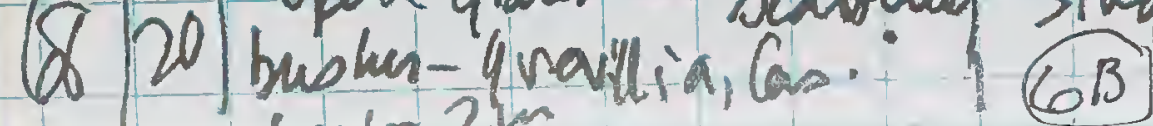

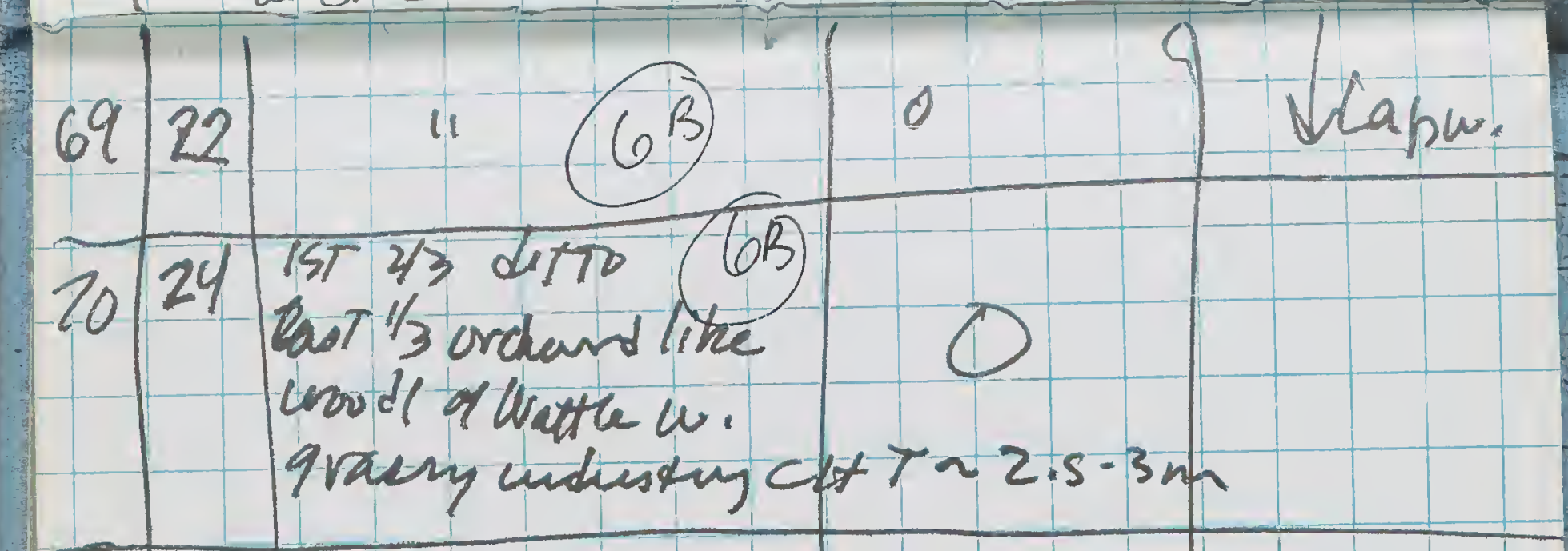

$1121 \quad 1130$ 2

$7220 \quad 11 \quad 30 \quad 0$

$7324: 11$ a terseng

7422 open wandos wood

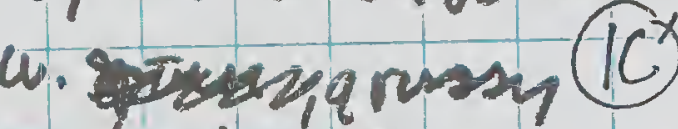

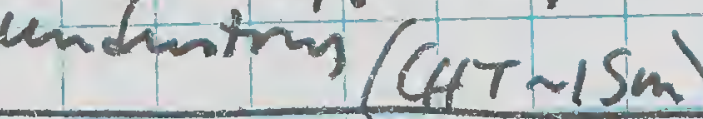

15.22 (1C)" but quas. spunso - muchdirtsum $15,1 / 3$ rd $-d_{1}+72$ last $2 / 3$ - no biy rees hut nore shonber Thich grass, Scaltured Sin, leatten to $-3 \mathrm{~m}$

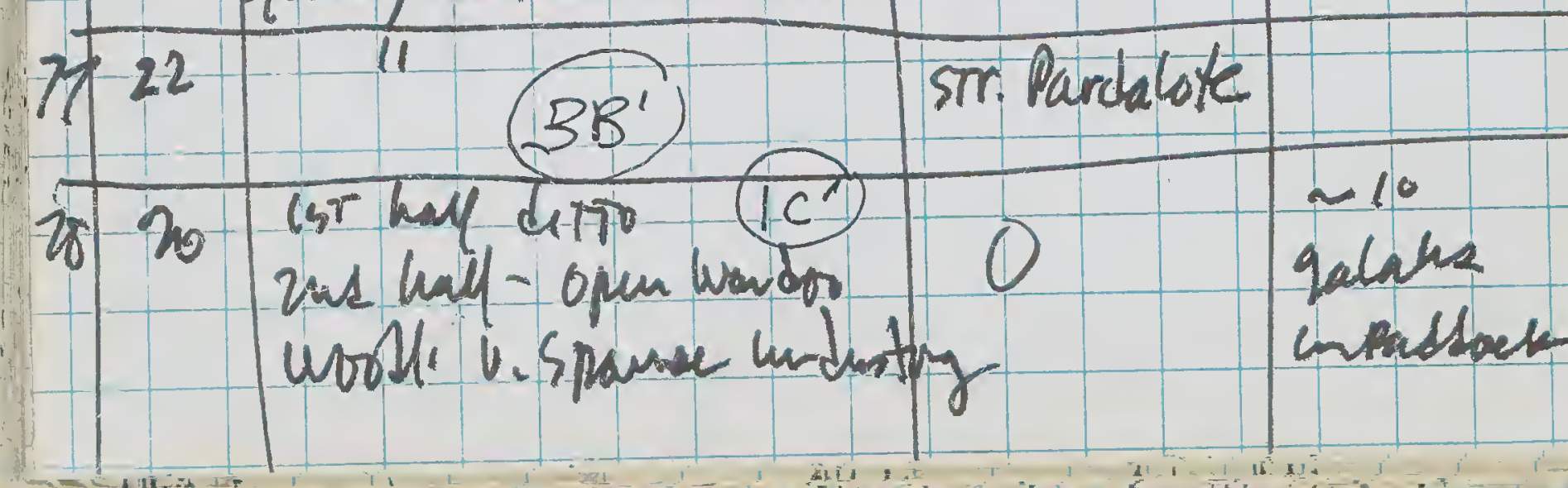




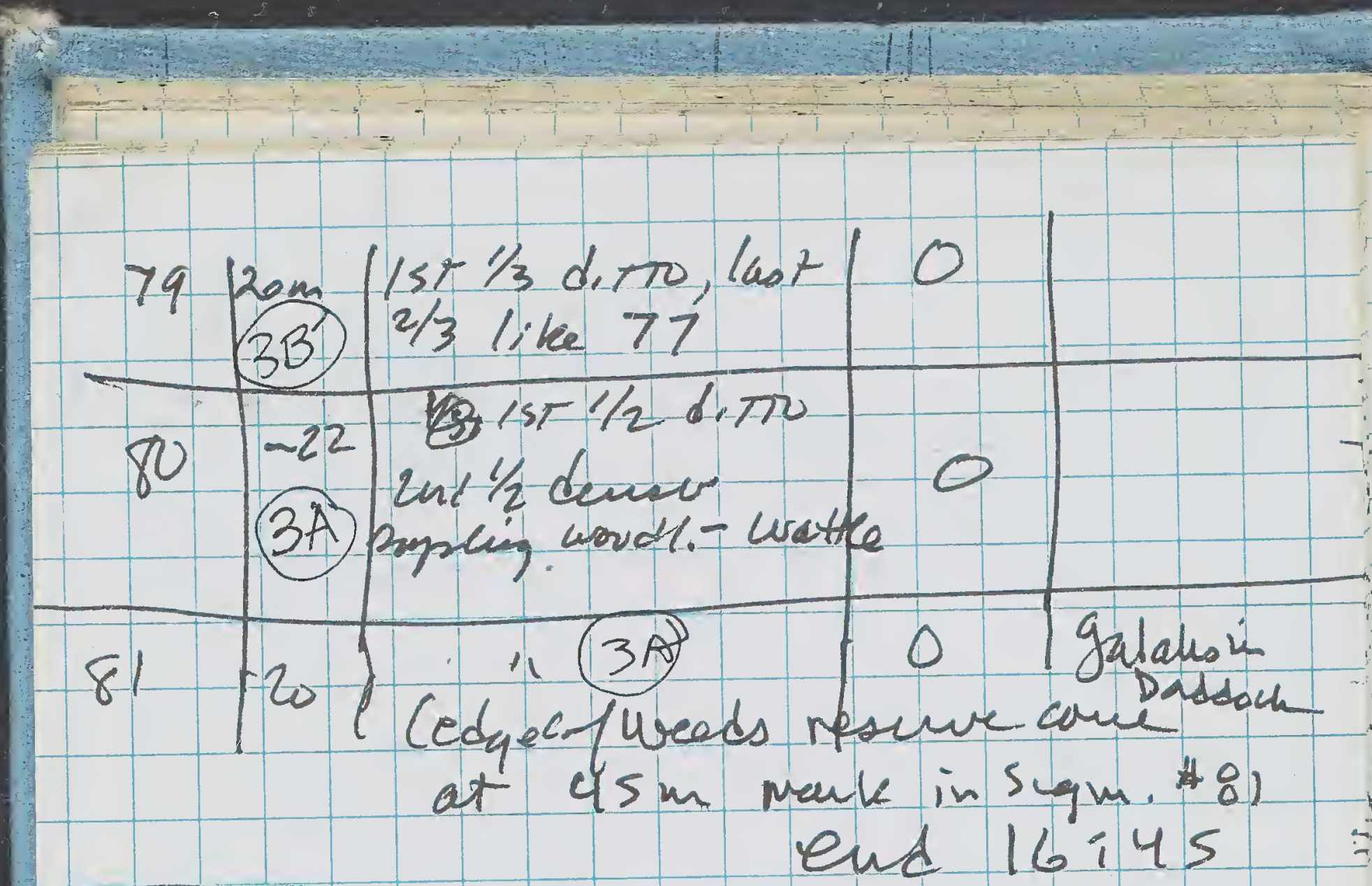

sat. 23 Jaby-loode, mild - vofors last night.

o8:w Buff-banded Rail feediz in garden at Csiko how - size of Quail delikerstely Pichin its way throrgh grass and avoud bases of husbes 8i15 Kangaro(pay?) in verge ot cover of Traquin Ret Buin Rd

Trausects Drvokappin-East Wandor wordeand Interior

$4 \cdot V \varepsilon a \quad c 25 m$

1 V. open wandro wosdl weebill

(10) Lq. Scattued trees, V. Low \&at $210-12 \mathrm{~m}$ grass-herb un duttry no den 20 of bresh

2 Sine, but smetreest lalahlotf Aswe saplimp r sm.hrushceups (IC) 3 ever une openthan

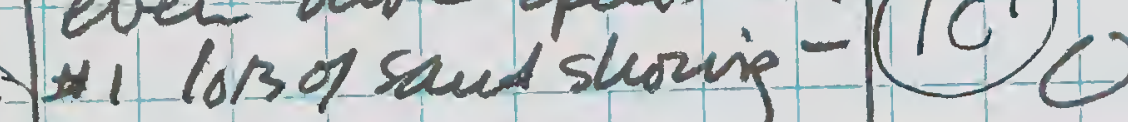
IC) wibly scathed qums

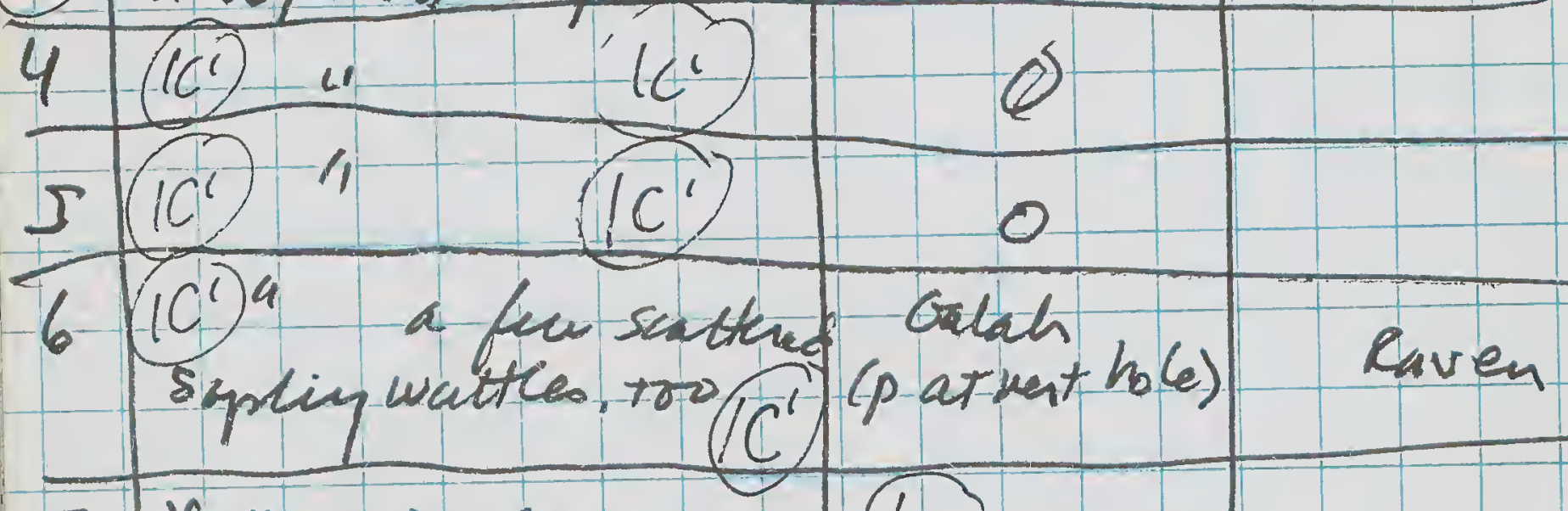

2 v. open w. avoly (lc) 0 (ic) V. few G Wardo-sm wartes, et.

8 mue la, trees tiket 4 galah (p)

9 Mob. Cunsity of i wandes (1B) (Goit Tolsm) w.open (mut thividurese) shrob/saplij hyer of mallec, Casuarira, Acucia eta.)

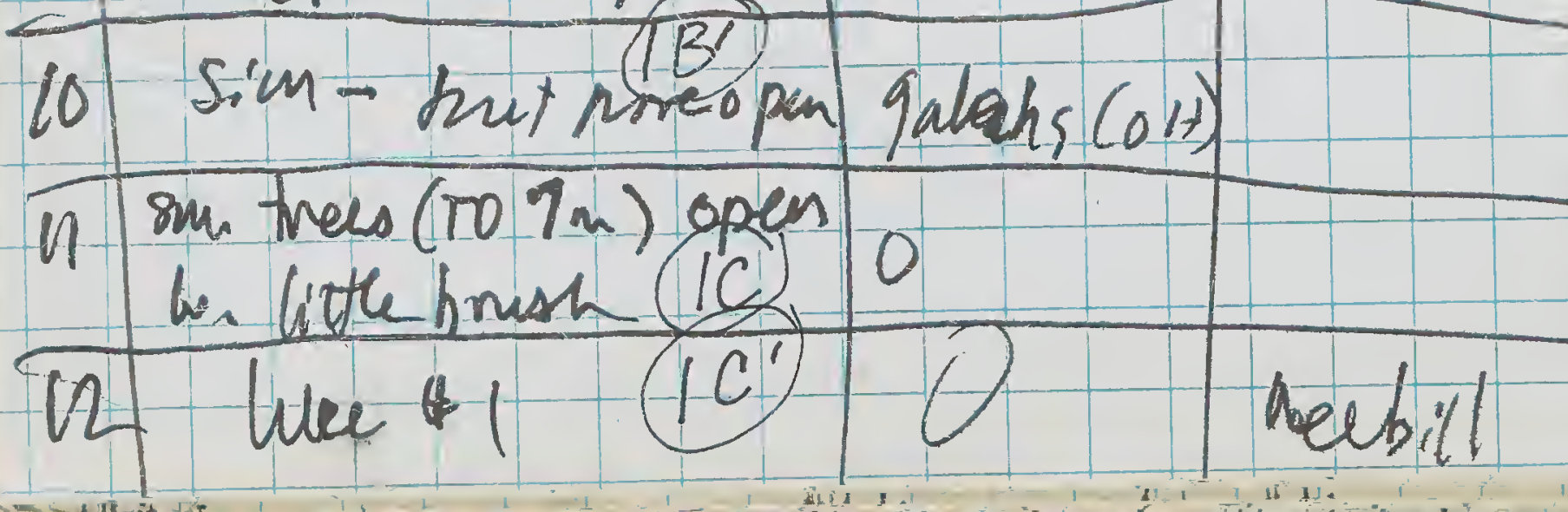
(16)

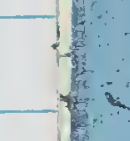




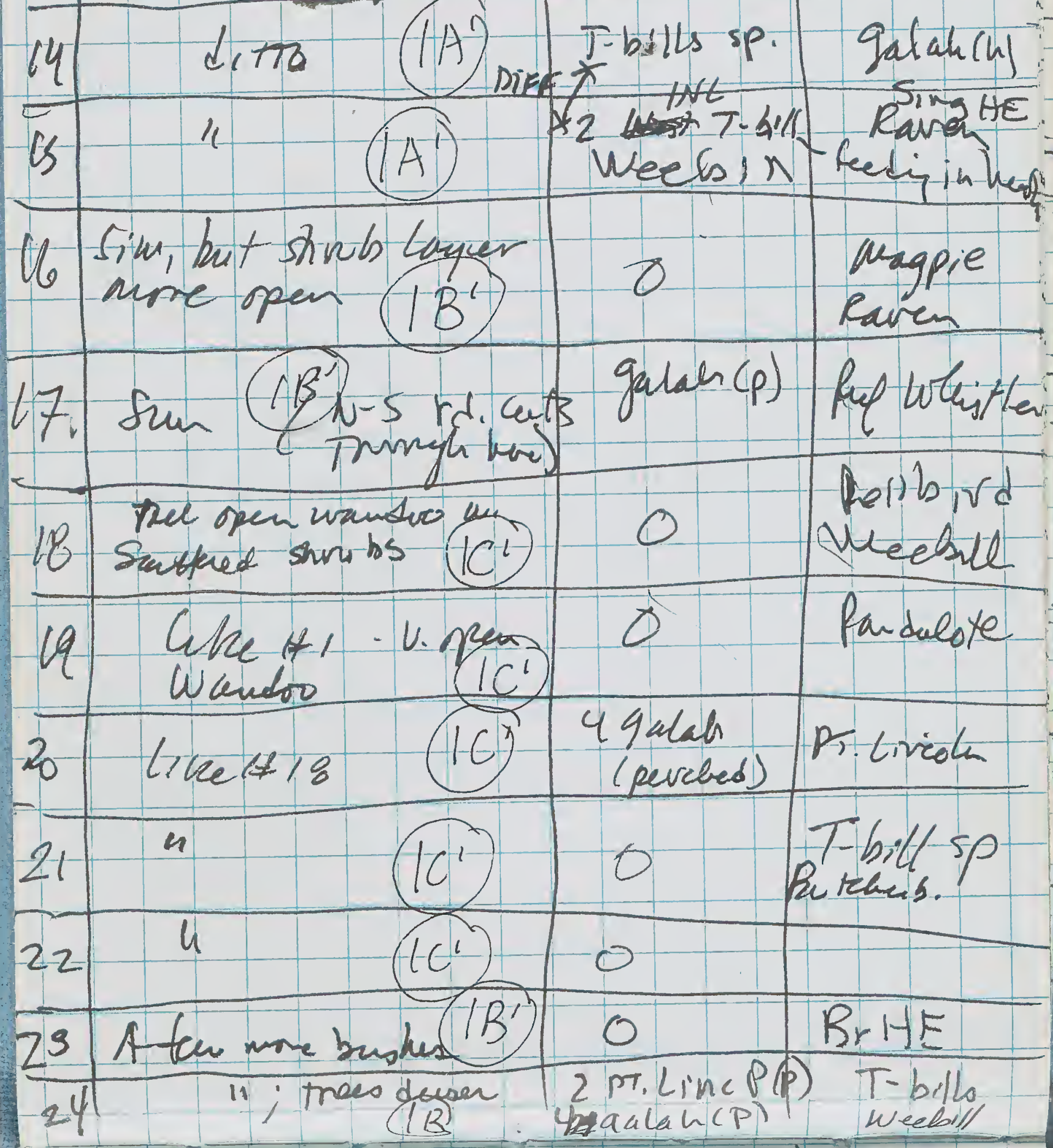

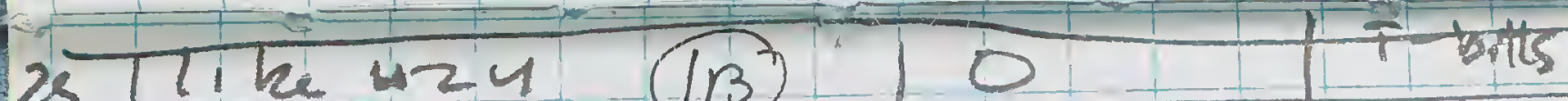

\begin{tabular}{|c|c|c|c|}
\hline 25 & Tike wzu (IB) & $\frac{0}{2(x)-1}$ & t-bill \\
\hline 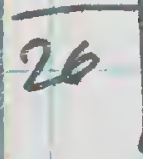 & 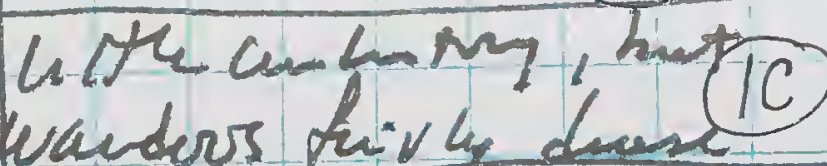 & 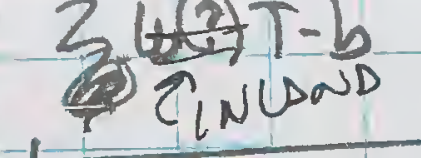 & Raven \\
\hline 27 & 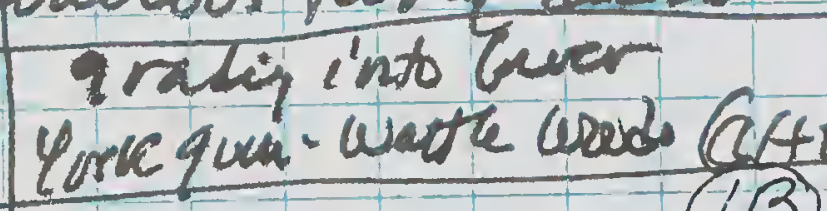 & $\begin{array}{l}40 \text { weebills } \\
\sim 8 \mathrm{~m})(10)\end{array}$ & \\
\hline 25 & 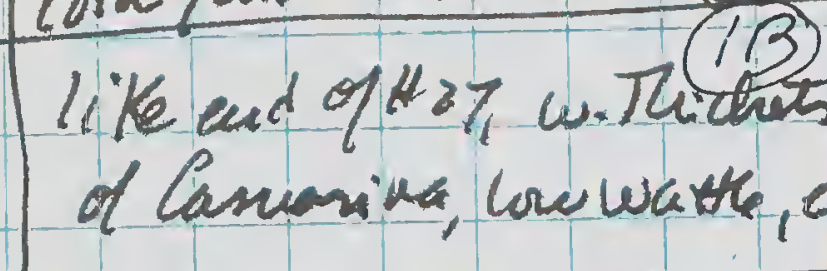 & $\begin{array}{l}\sim 6 \text { cureebil } \\
\text { (HE Tipso) } \\
\text { T. Cannanina) }\end{array}$ & $\begin{array}{l}\text { Pr.cince } \\
\text { gulahs }\end{array}$ \\
\hline 29 & 11 (B) & T-bill sp & Belbird \\
\hline
\end{tabular}

30

"orchare" type lowwendl.

of wattle, Shuchlurod; open.

of wattle, shudal word; ofen 0

sunubs

31

(3C)

31 (3C) $\begin{aligned} & \text { arfantail } \\ & \sim 4 \text { Y }-R T-b i H\end{aligned}$

32 "3c ends at P.C.nc.P.

eque of open wandoo worde.

35 like 32 , hut w. a

- (C) Opar vander

34 W. v. Scutenes shrubs

galahs (F)

harshchewte

38

8

36

(12) 0

BaAE 


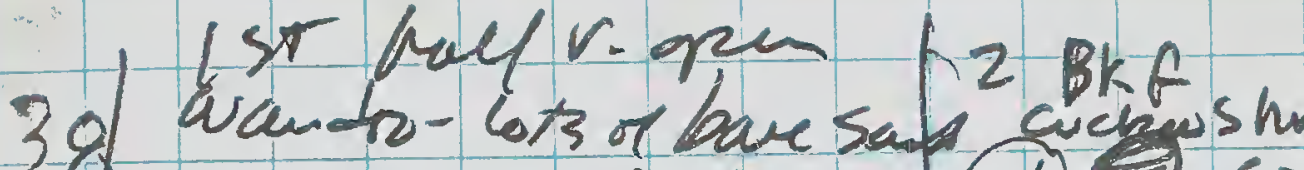

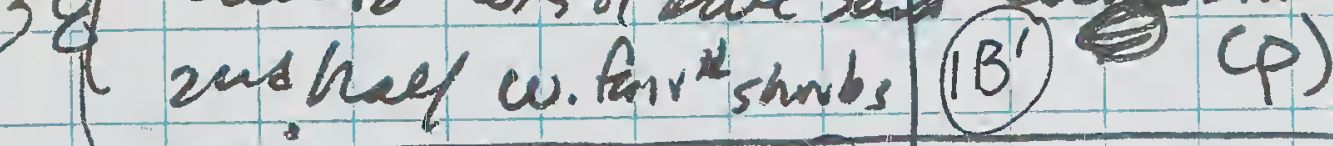
Wandson w. (B) 2 heelsill

34 Sapizizedrees I Fautal

34 Scasend bneohdemps Red-C Robind Rvtt

H.upurtavdo

- verdl.

Qu.

41 stad. surbs * sapl.

$42 \quad 11 \quad\left(1 c^{1}\right.$

43.1 last pont \& $1 B^{\prime}$ das 4. Casuring Theher toth sibe

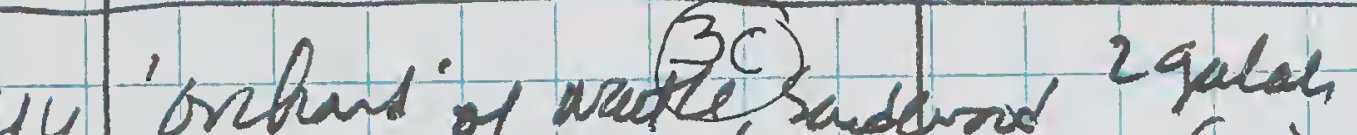
44 openqaryuntinto $(0 A)$

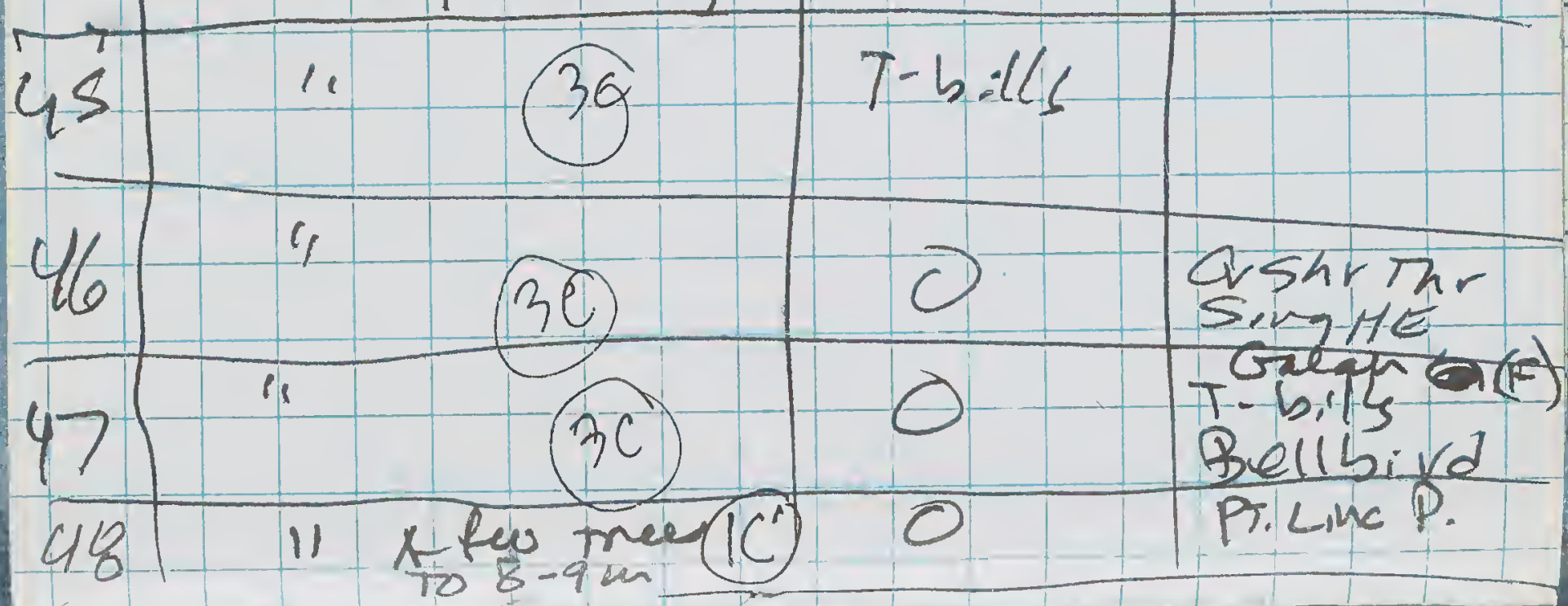

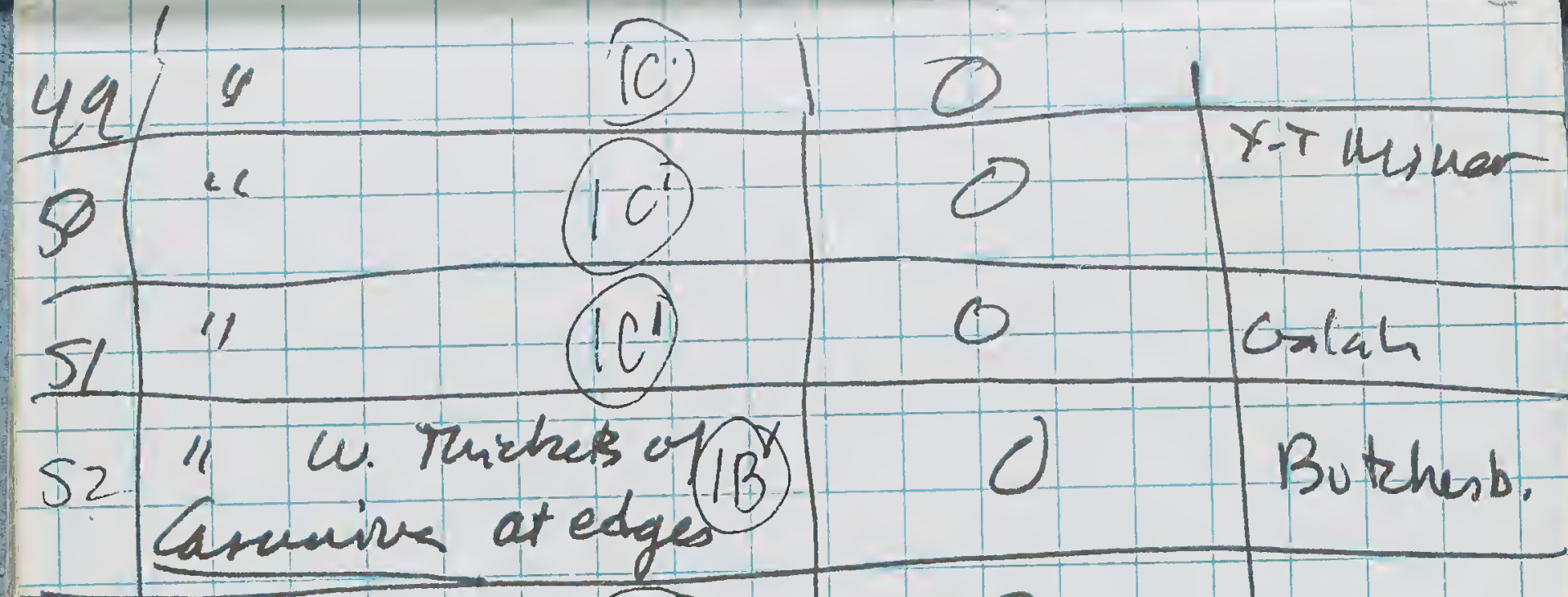

52

"1

53

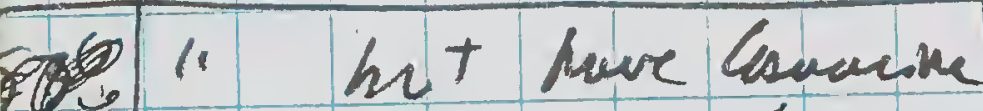

54 $1 B$

0

Thills

Pi Linee Galah

3a is loy like 53

ss last $1 / 2$ qua besinta Waudro voon $x$

$1 B^{\prime}$

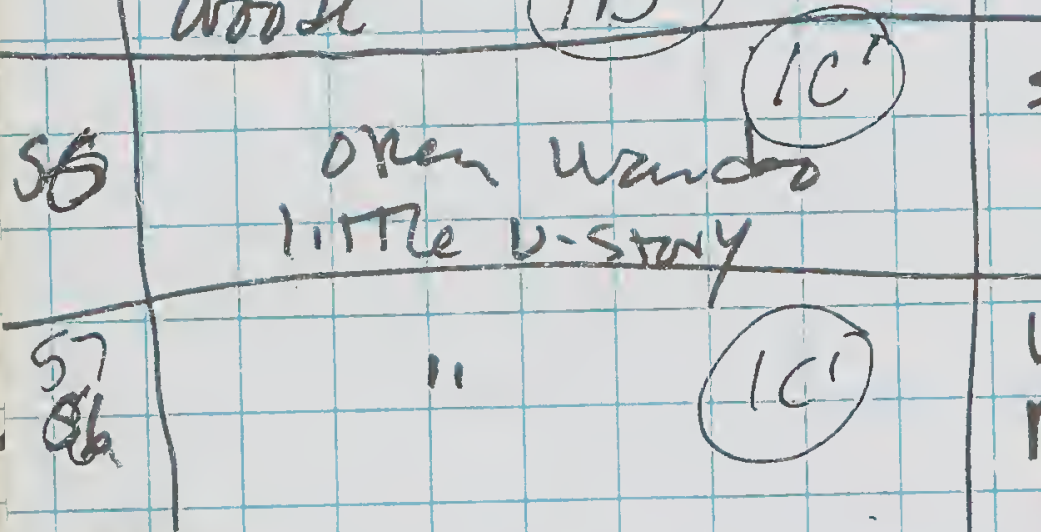

w. vanble BrHE

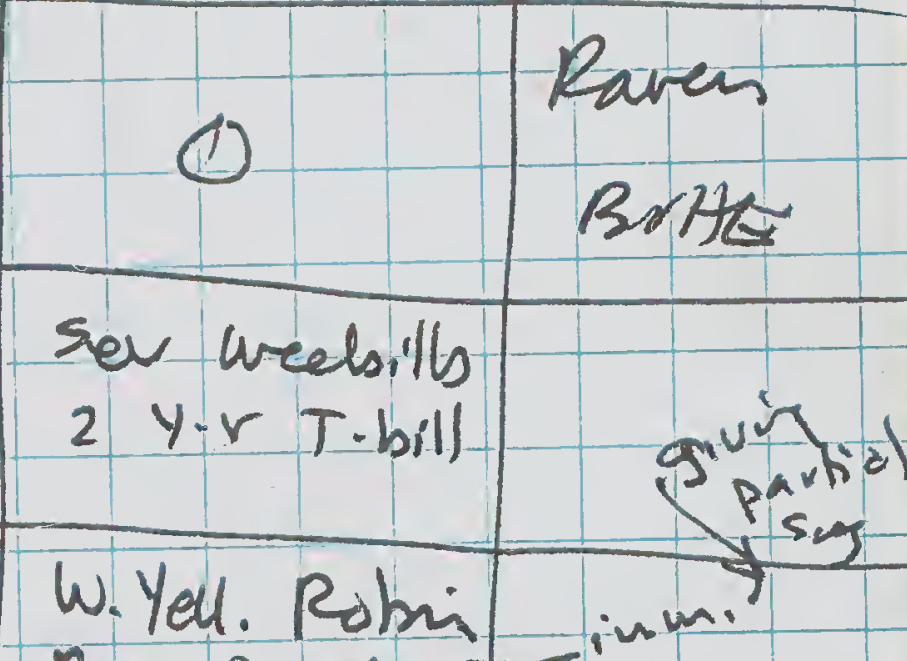

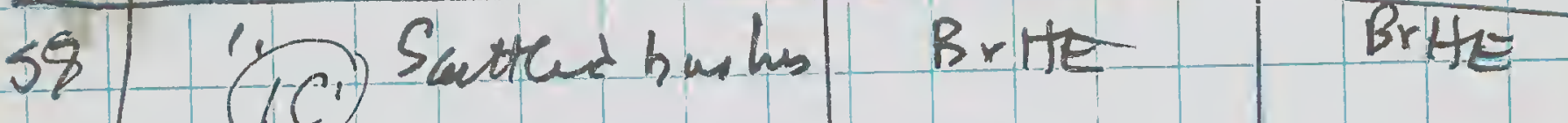

\begin{tabular}{|c|c|c|c|c|}
\hline 589 & & (ci) & 0 & \\
\hline $\operatorname{sg} 60$ & 11 & $(1,1)$ & treeb.it & Brte \\
\hline$d$ & y & $\begin{array}{l}\text { wore hushes } \\
\text { and sapliogs }\end{array}$ & ORR.CADL" & rans,id \\
\hline 68 & & 11 & 0 & \\
\hline
\end{tabular}


tho 18

is open Wandoo-Ywk J. Wiluter

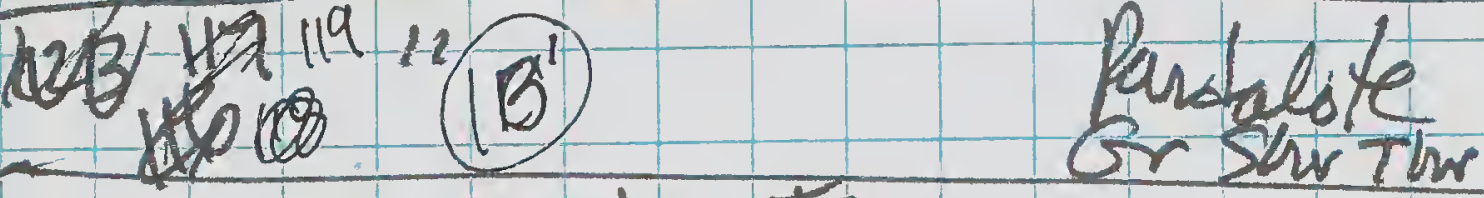

1 stake $\sqrt{500}$

- I put orange flog every som fum J500 to the place whue the Tus or wistlers were Digin, $200 \mathrm{~m}$ NW of here

BPA W. Weath to the ( O

12. $\operatorname{lef}(S E)$ (B'

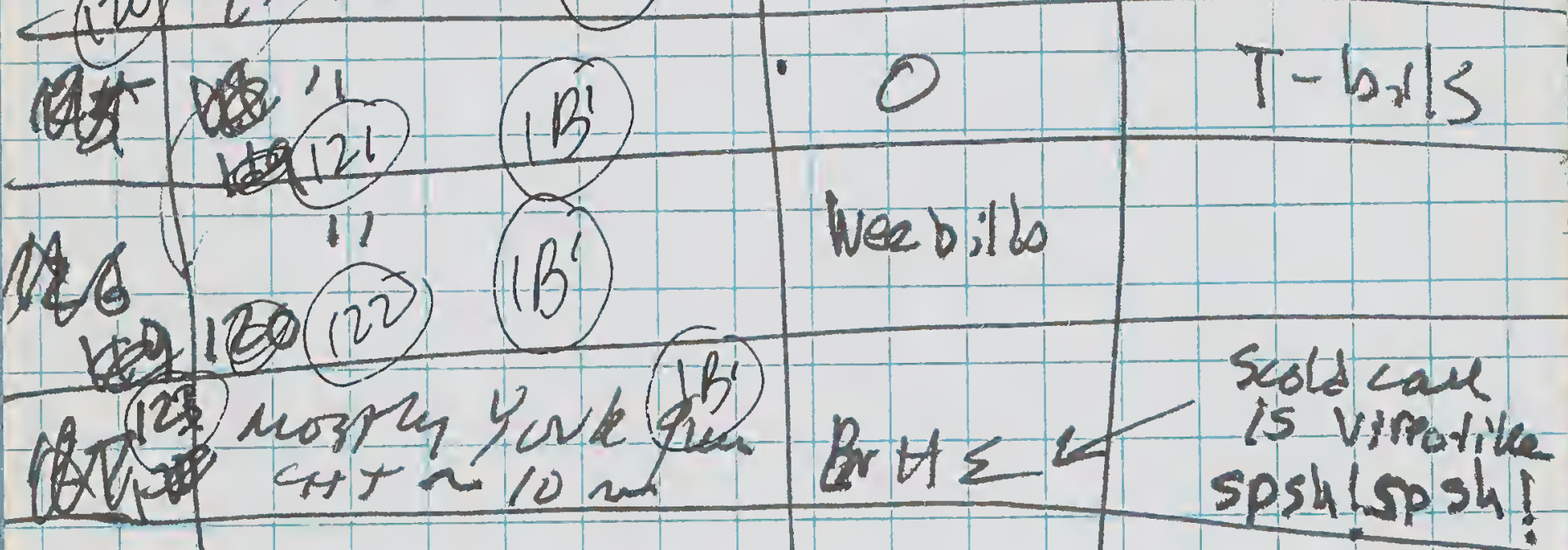

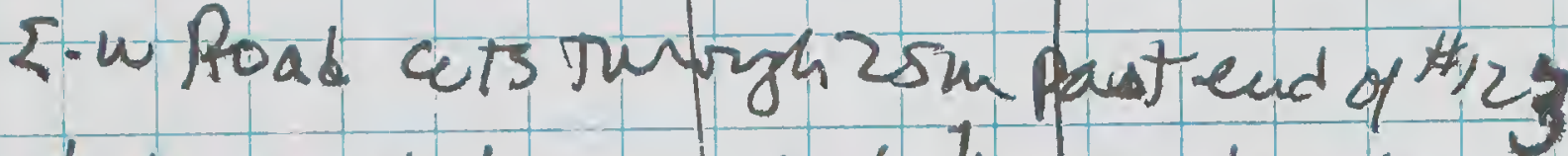

I tumed west into hesy heak

1170 faircy dese 5C su. Bablibzo

Q1 Gusuaina, qrevittea

heath CHT To 2-2.5m

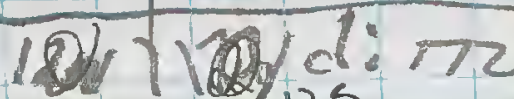

(50) $c \in=20 w)$

$186(29)$

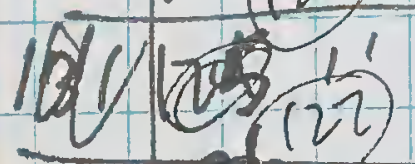

(5C) BrHE

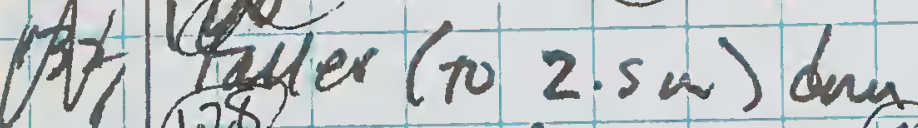
IV) 25 duse Caswarin (5A)

o

(50)
2.5w) den 5 .

0300129

(48) 0

CQN

U.

(6) 1300

ubel 5. side of path like this 5 D

oedN. Side like 128

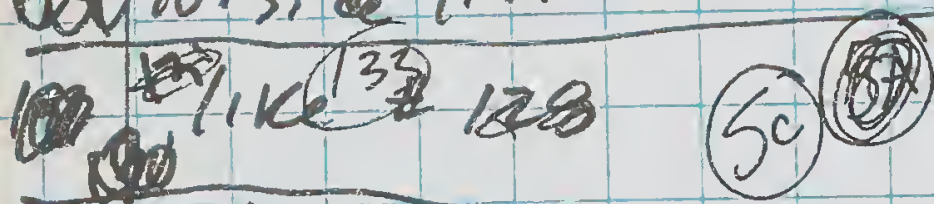

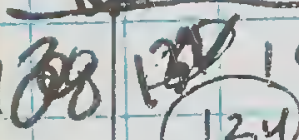

10 inge

1030 (135

148 迎

( $132+132)$

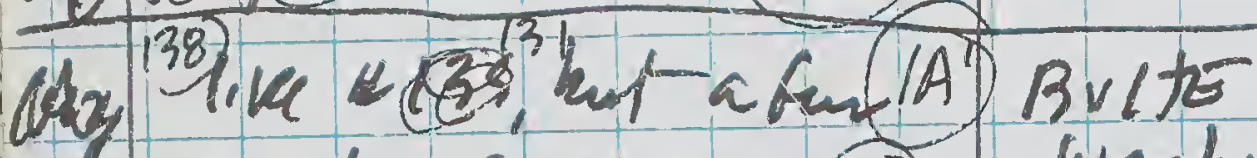

(20) cuandor S. of rd

(39)

(|A|) Brite

$\operatorname{Br} H E x$

2 BuHE $x$ DIFF

ZBrHE $x$

BNAE \DIFF

We nuar dircrse tree -

10 Shub-grass Mix IA

140 a CAt $\sim 6 m$

QP Sho The 


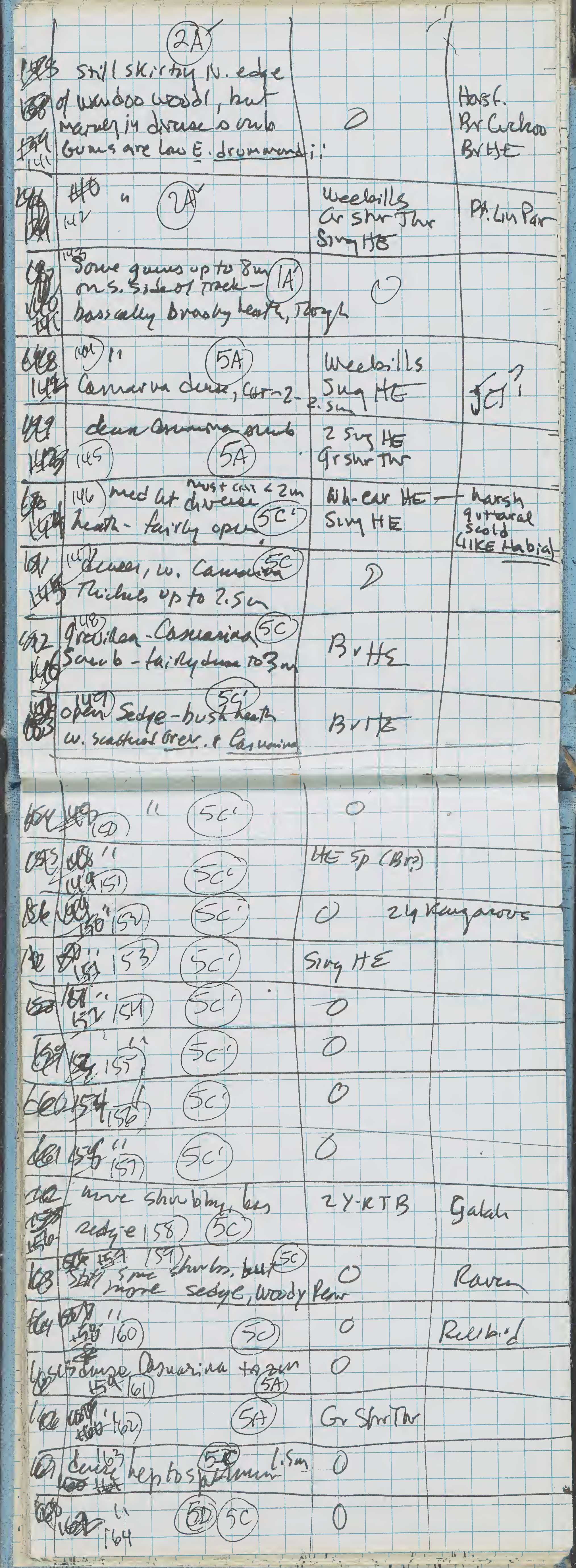


83 duly Dusopoppin日 (cout'd) (1) Varied shmelan w. Casruma to $3 \mathrm{~m}$ olet Canma-wathe

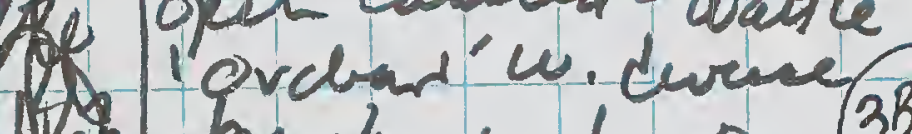

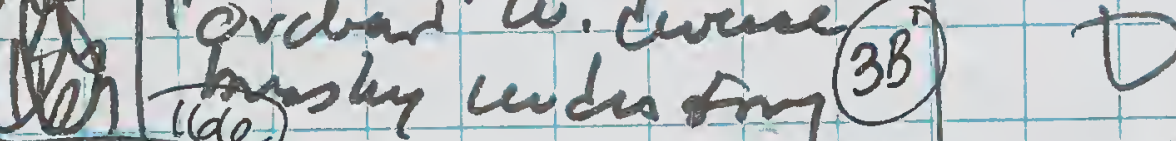

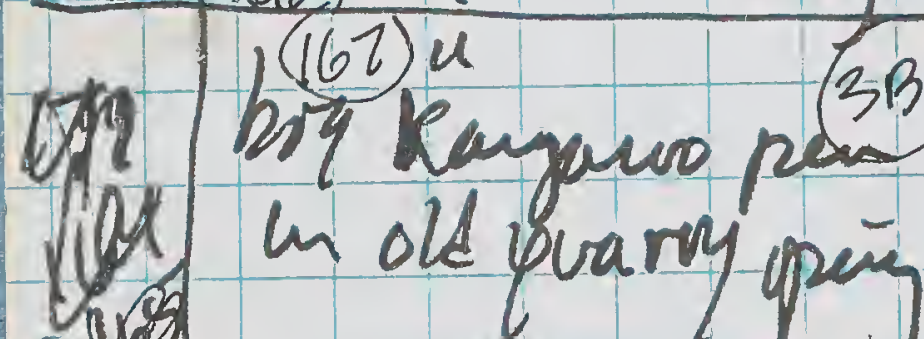

IIlक surewate - Dat Lasacinia leode 186) CHT $\sim 5-6 \sum^{3 B}$

16) (198)

(nis

$4 \sqrt{10}$

eal

cuds atbavis w. gam unase

T. 11 .

15e Gen Wanda uror de.

(16) Scattered bum kn a sapl.

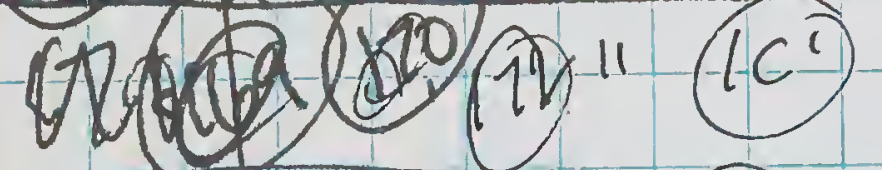

101.2

7 (113

1.

aic

(16)

o

1

(x)

(1C)

0 


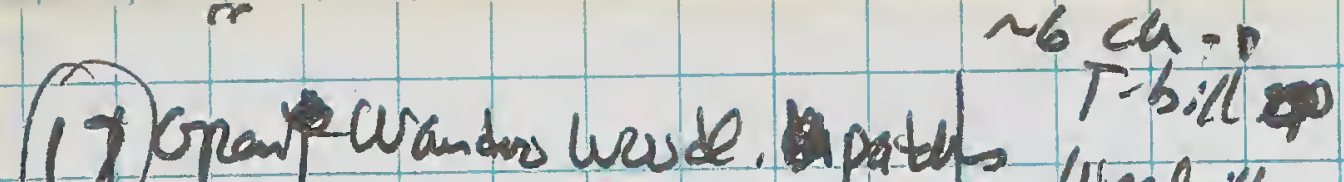
advere brash but Wreball

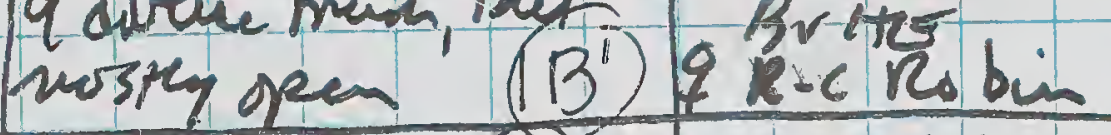

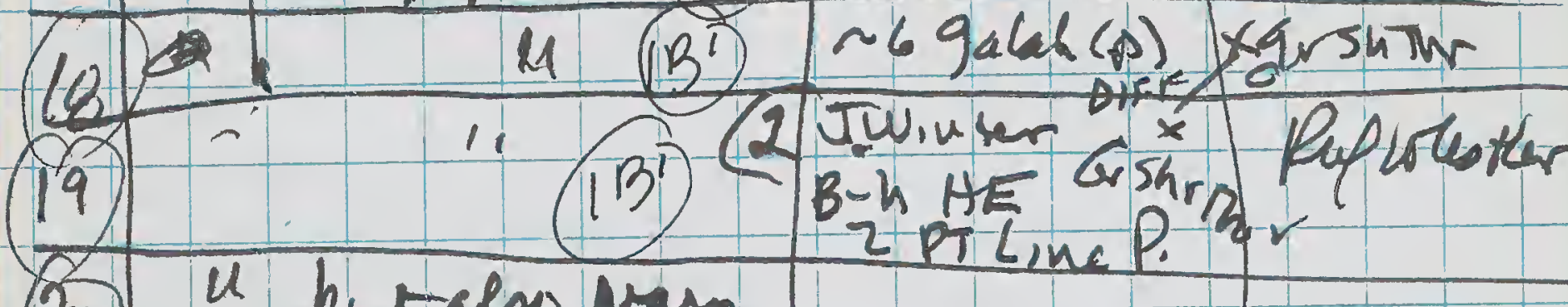

(20) U hut also May wader sopey $13^{\circ}$ F.80 mastres hera

21 "but far ent (IB) 0 a. lop of Caswariad dupp

(22) like $\$ 19$ (1B) Dave

23) ISV kay" ; ind hate (18')

2) W. smoreangradi mot 0

- bush suvama 24 Weath $1 .-1.5$ mg Br Brtse + grshrtw to $2.5 \mathrm{~N} 3 \mathrm{~m}$

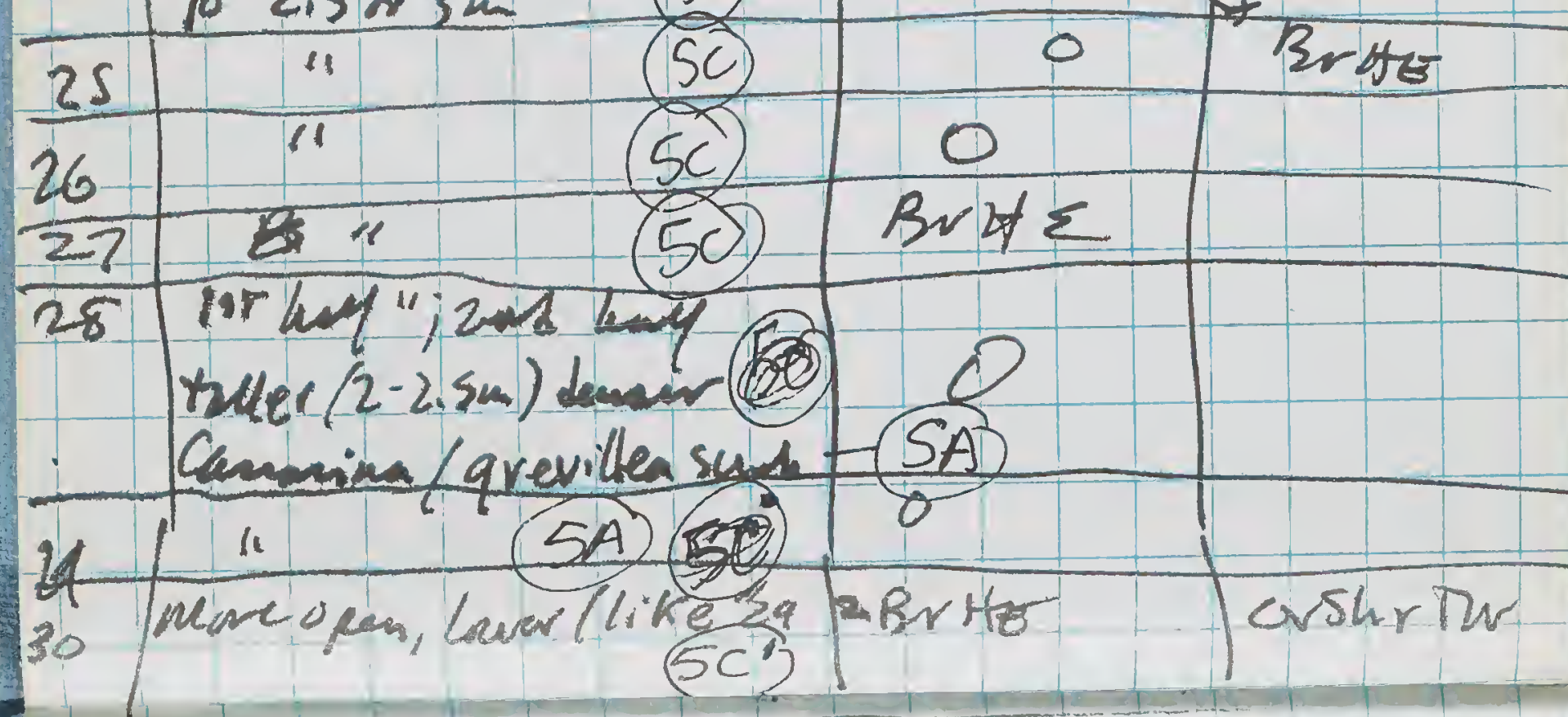

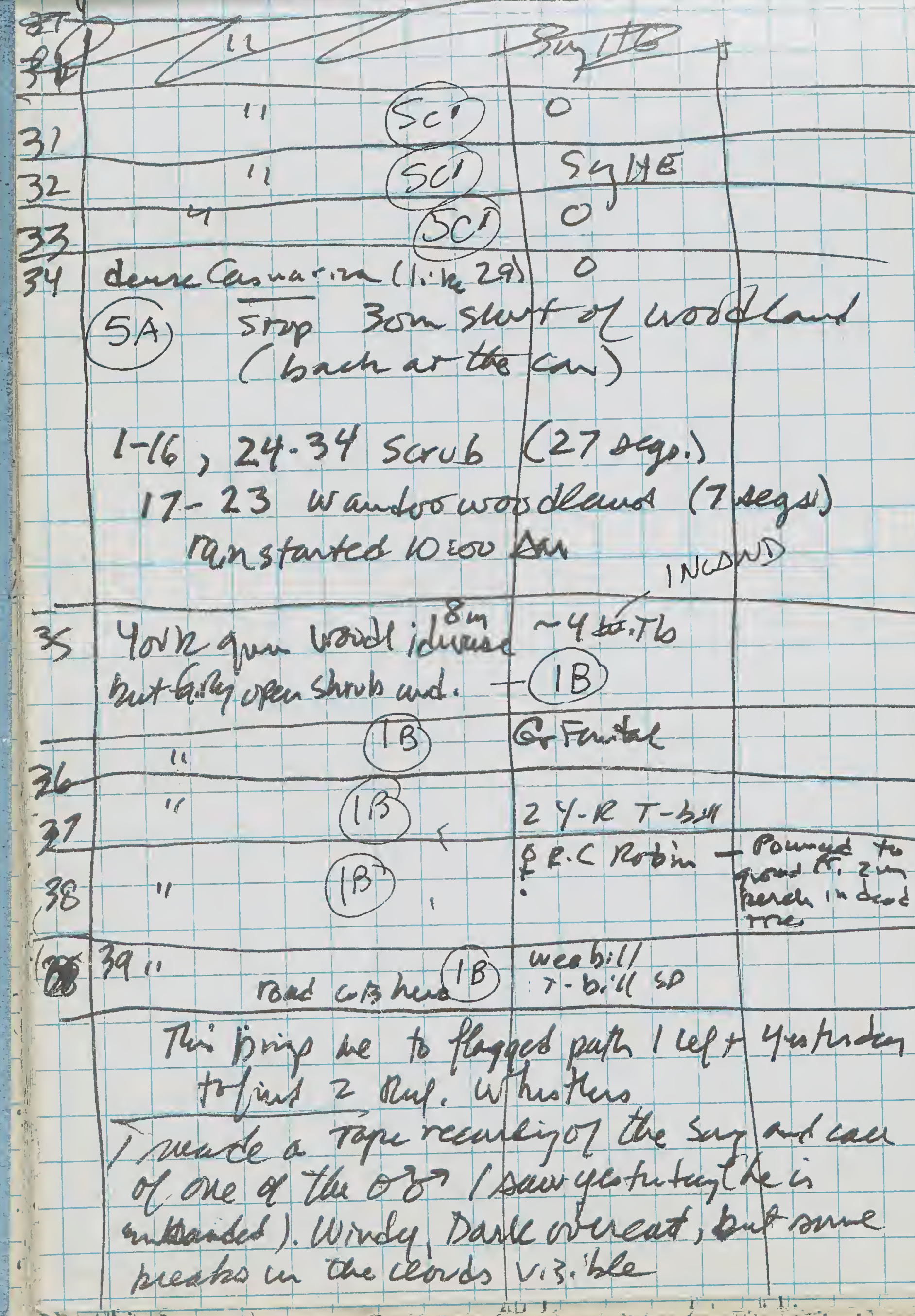


RAw d kizh whas ael is Inly - coot, clear, but w. gund Foy, clem

o 7ius S.end Duvoroppin East interior

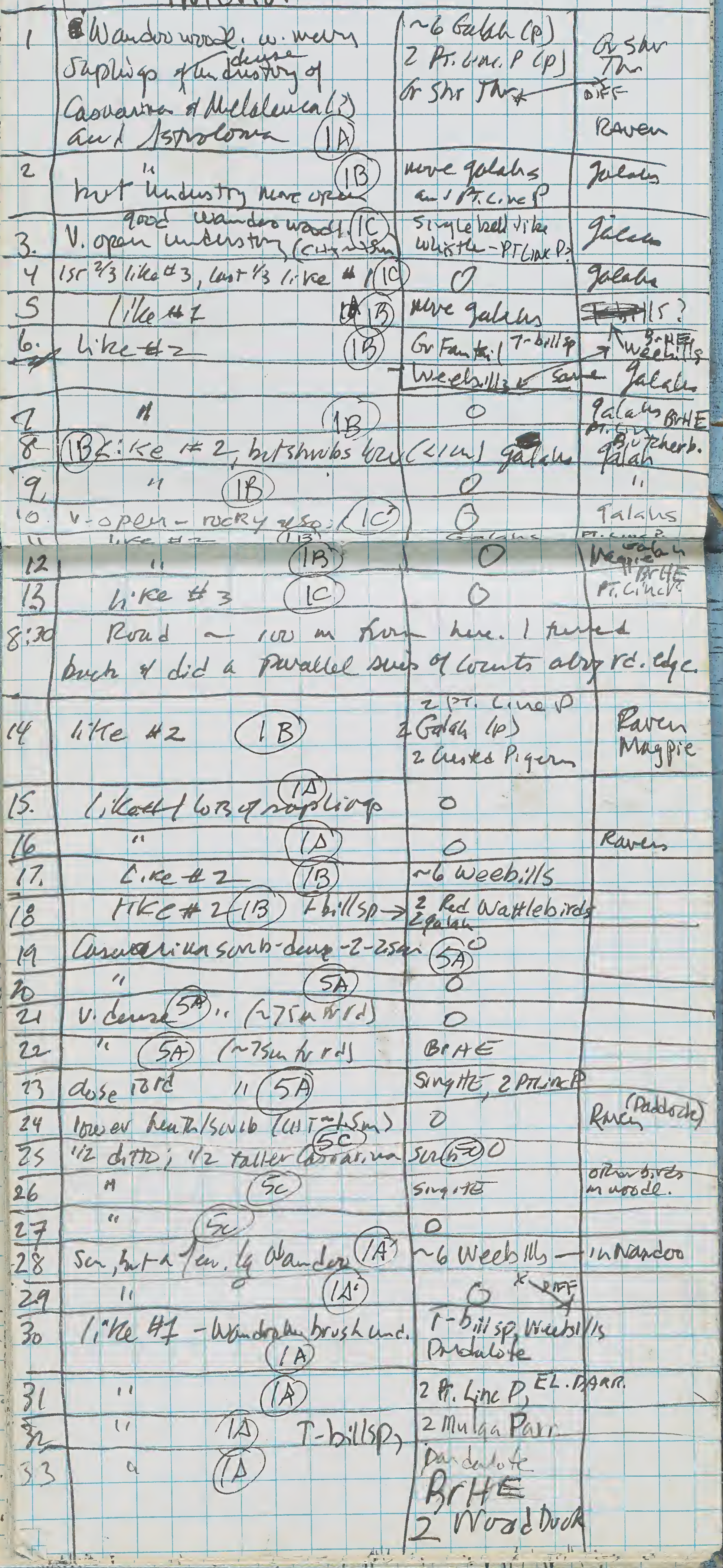


535 sim bet more brushre cot $l-1.5 \mathrm{~m})$

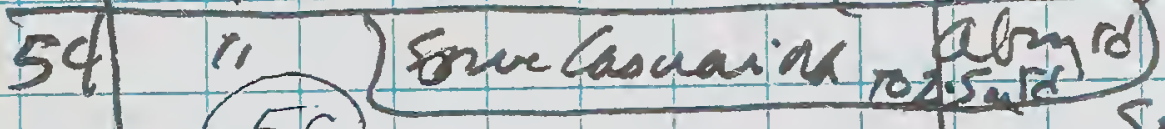

(5c) singtte

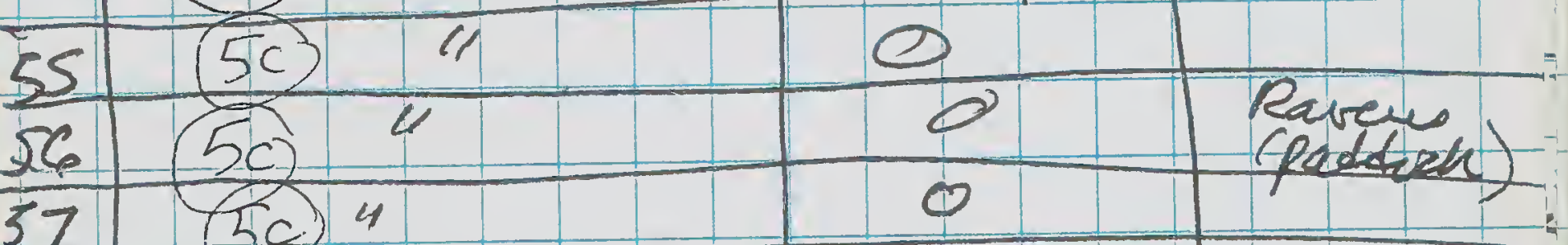

58 (5c) a 0

39 157/24tto; 2nd hat wo taller

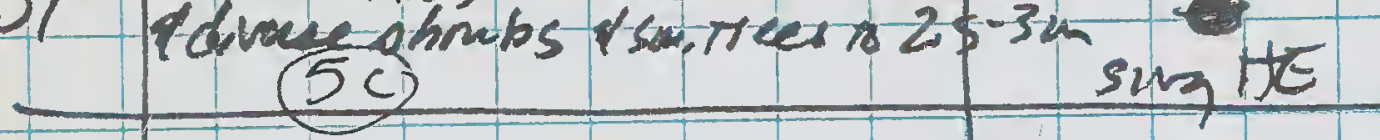

60 (50) $1 . \quad$ P. Live.

(5c) 110 galah paddode?

CI 15T bral "; 2nd bay (5A)

derse Thichts of Cas grov, ebe.

62 like bot bue H 161 (5A)

$\begin{array}{lll}63 & & \end{array}$

64 low qum wovdend - (23)

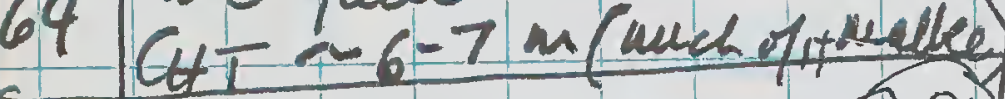

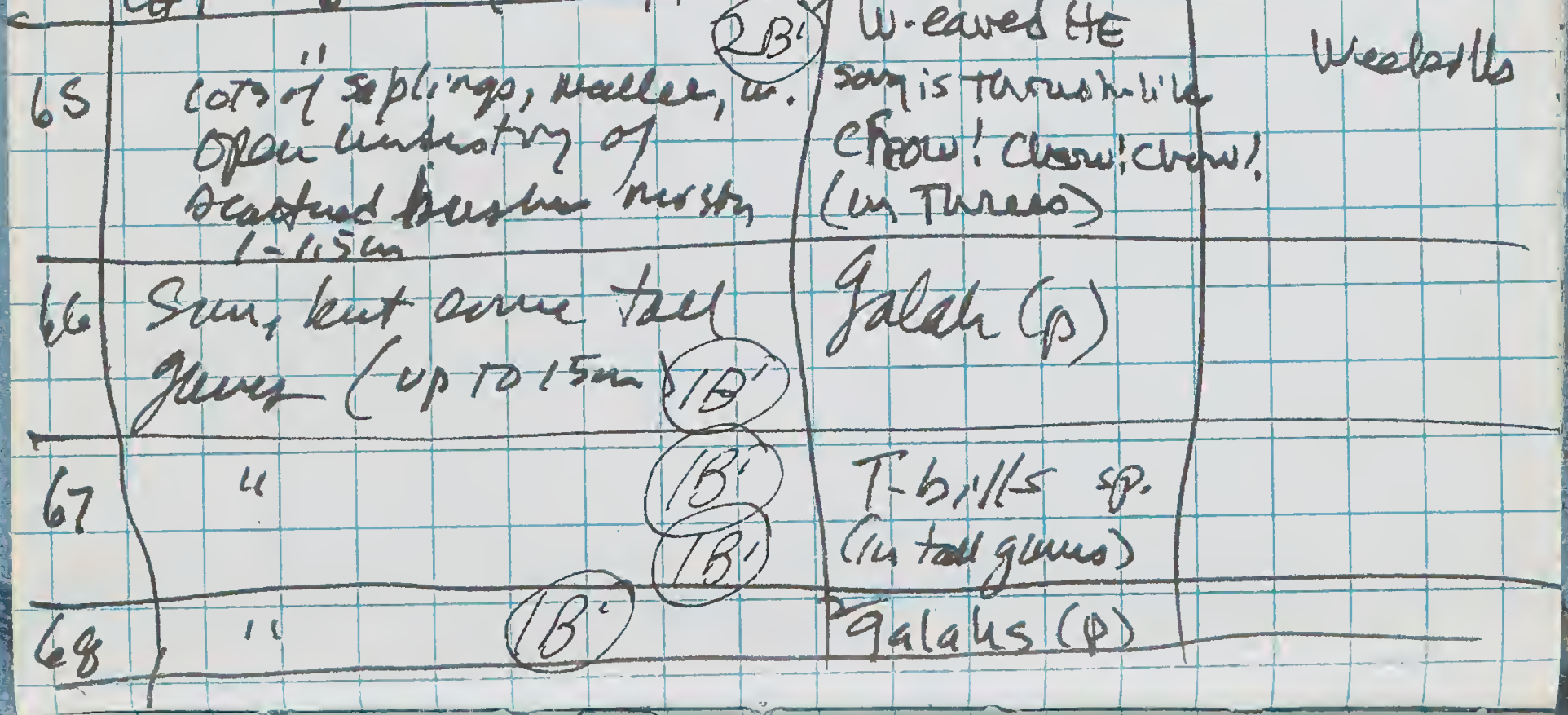

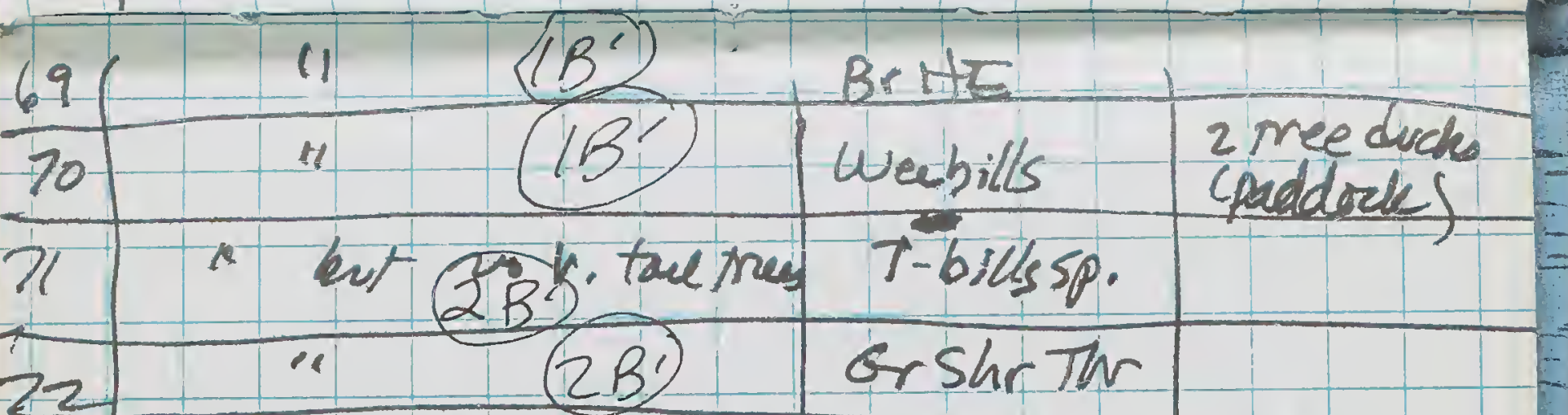

73 atra y-s a qradizinf

pathycensing (2B) 0

Iy spen wands worde. wC

Scathed bushor-lasiqgars

10:25 SE corver 1 reun $-200 \mathrm{~m}$ abed 1

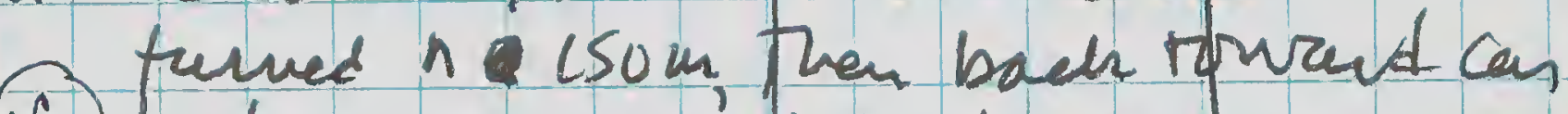
(C) to do a suies of 'iuland' segjues

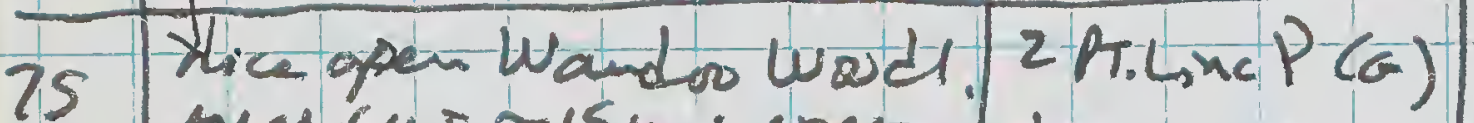

75 masut r $15 \mathrm{~m}$ jopen weeb;lls

Fref Whiter

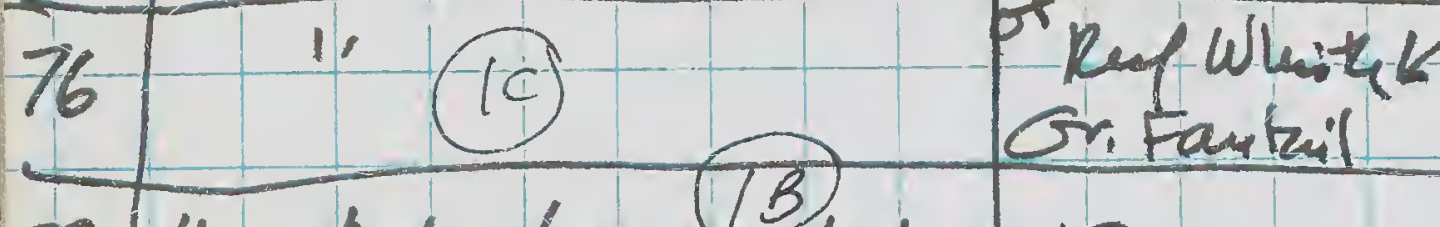

77 " hat deresees shinb leyer dom. by Astrolonua

28 lower CHFT ( $\sim 4-5 m)$ aradiy ZPTL,us $P(p)$ T2B)

79 Malfel sublobrush

Crshrothor

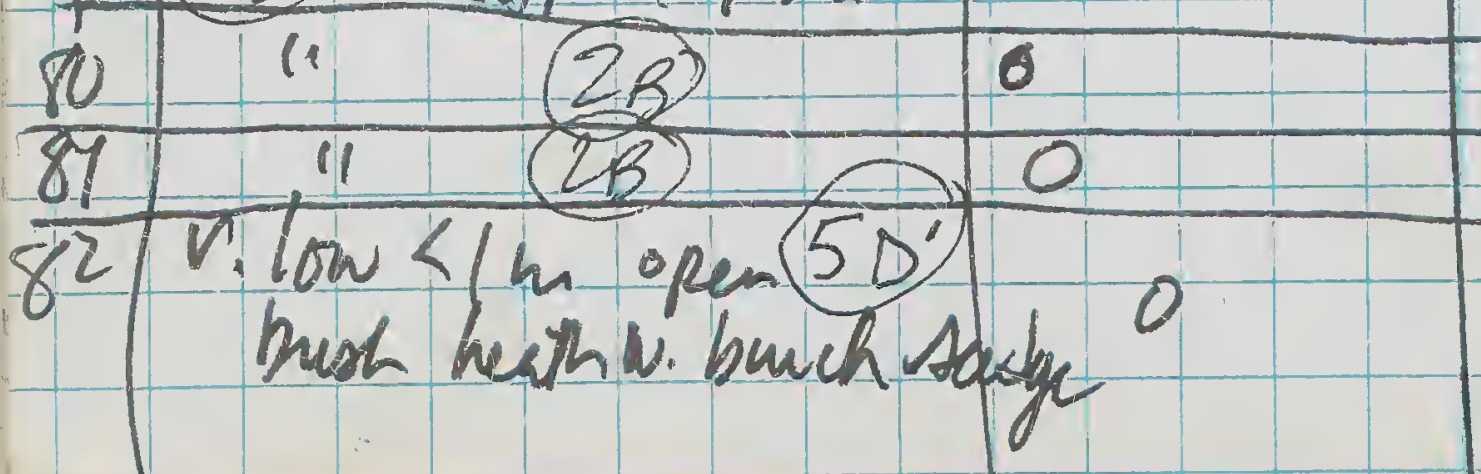

Th $1+$

Pardalore 


\section{(2A)}

83) malleew fairy dever

$2 B r H E \sqrt[T A]{4^{T}}$

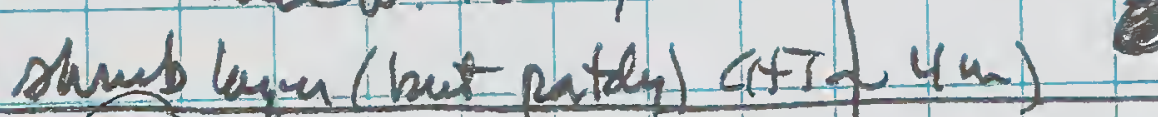

\section{$\frac{89}{86}$}

89 (2B) \& bitureopen 0

$85(2 B) "$ " 11100

$87 \frac{2 B}{2 B}{ }^{11} \cdot 11 \quad B r+E$

88 (2B) B 700 stik her $O$

(86) ver mume open, pat (2B)

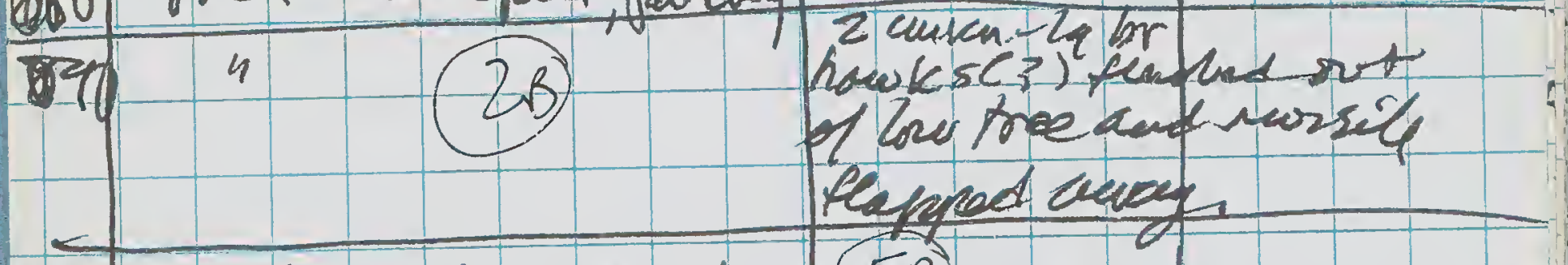

Q Tar deuse buesh of $-5 B$

Melaleueq Ssrolena,

Watce exc. $(\mathrm{Cl} T \mathrm{~T} \sim 2.5 \mathrm{~m})$

on tou Wandoo worde. PT.Linc.P.

Cats 12.rsm open galeh

brush in mideston (B)

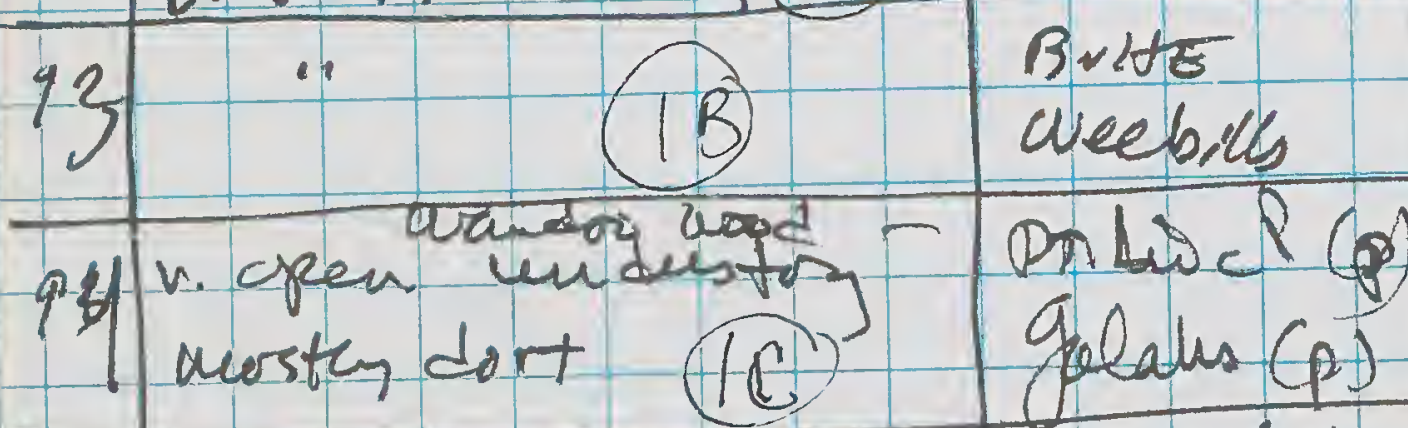

Brite

Buts T-3rib

Sm, but tair \& sectent weoplly

burbes to $2.5 \mathrm{~cm}$ higl(B) thillsp $(y-R$

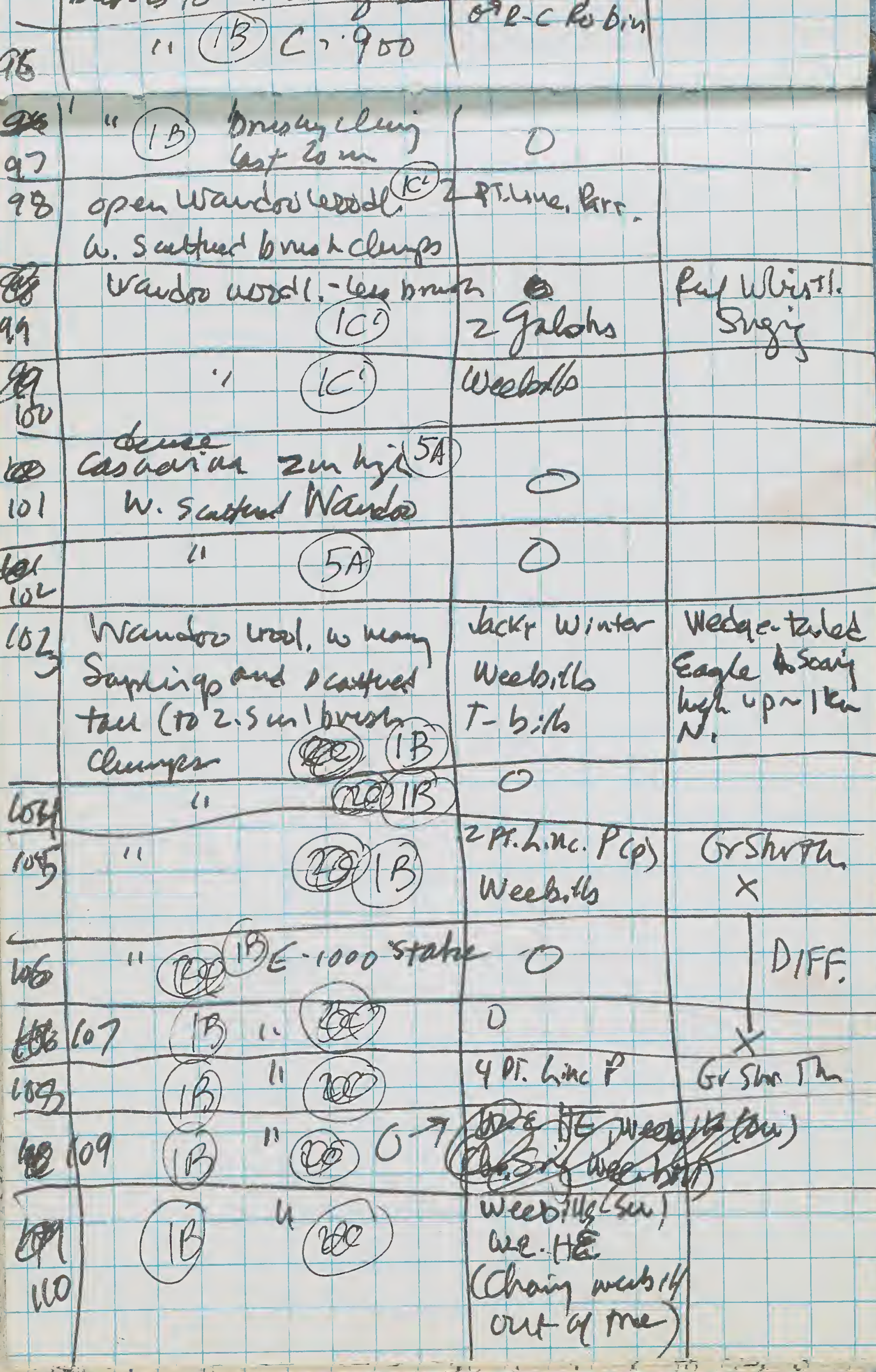




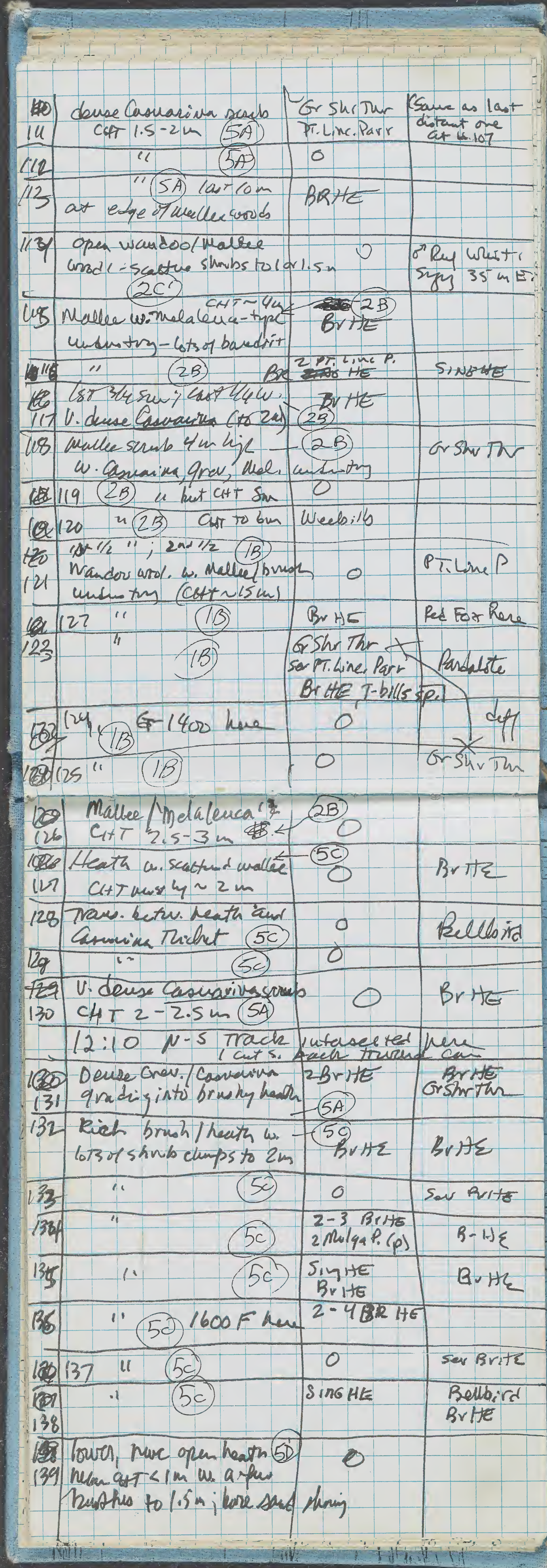


Sim but a peul

I4) ocatuded grevillea to $3 \mathrm{~m}$

11 10 c)

14. kopugon scabriusos blater

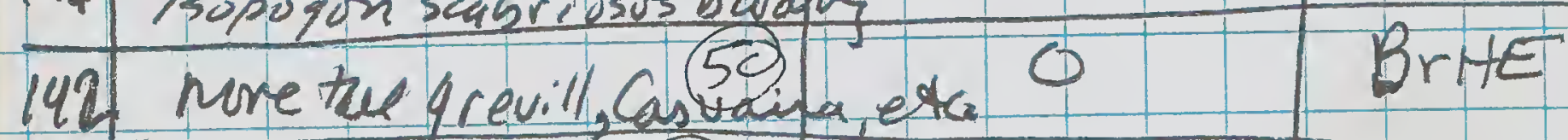

" 50

4 斯

(5)

Mnyt taccarom. of

149 oth ohubsto $5-6$ ar

\begin{tabular}{|c|c|c|c|}
\hline \\
\hline 1465 & 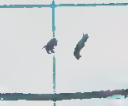 & $(4 A)$ & 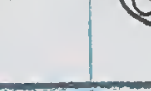 \\
\hline 108 & $\omega$ & $(\mathbb{A})$ & \\
\hline
\end{tabular}

\begin{tabular}{l|l} 
Sinc HE & BrHE \\
&
\end{tabular}

W.E HE

B $P$ HE

BVHE

P.lira.Pan (P)

Brtte

2incthombill Butte

2 PLLUna. P. (G)

smg tse

BTHE sen Weatail

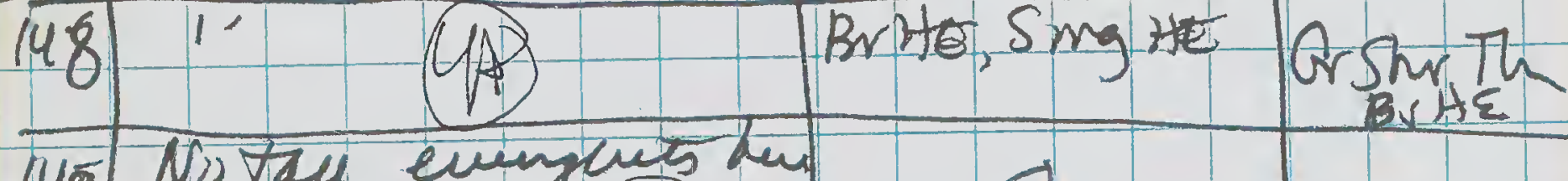

140 Notal evinglus tur

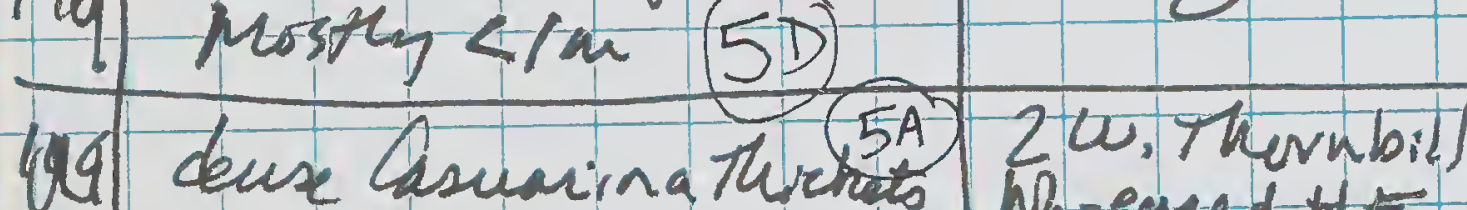

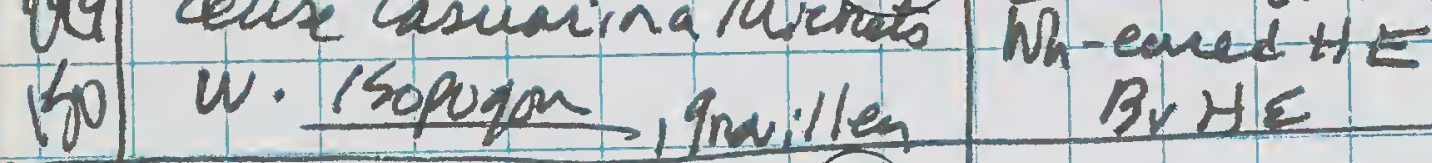

1S4 dense Curmmin Thist - 2-2.sm 0

152

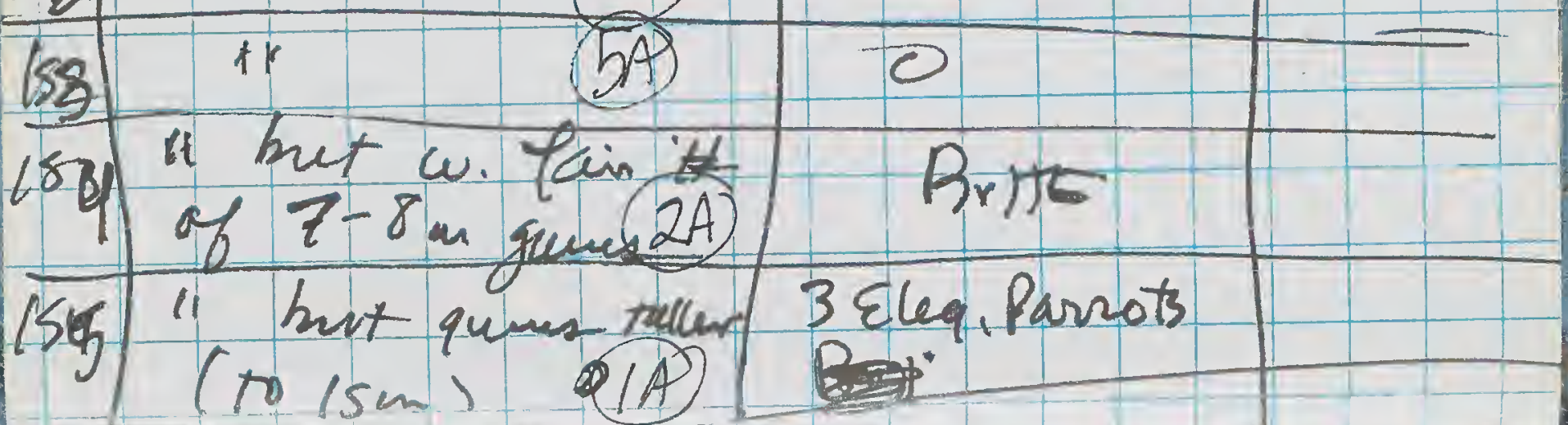

156. Wardar wos du u. (IA)

farly deus tasvaiver undustory

2 galah. (p) BrHE

BACK TO CAR at" 12:50 $P(C$, wild, IT. breeze

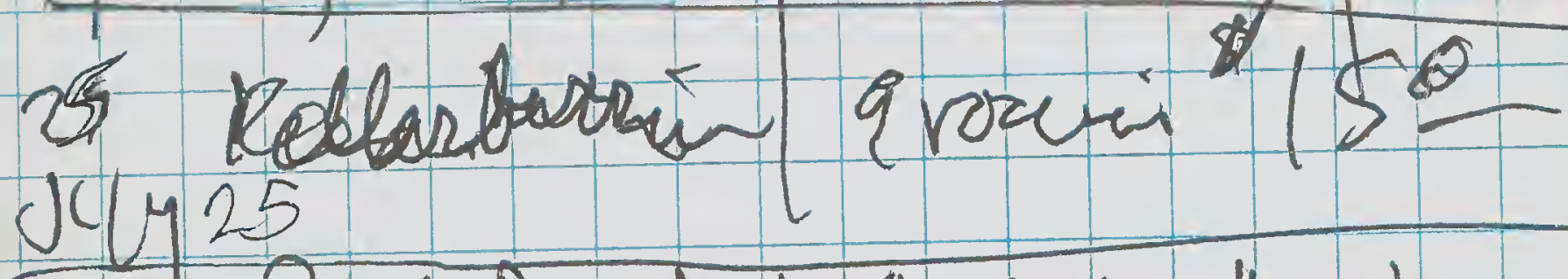

D.M. Pied Butzherbird cael- kash vatal "Boinne" Inapine a 2olb-R. Sided tawkes call

N.edge'Weeds Reserve' - Edqe a Interior seqs

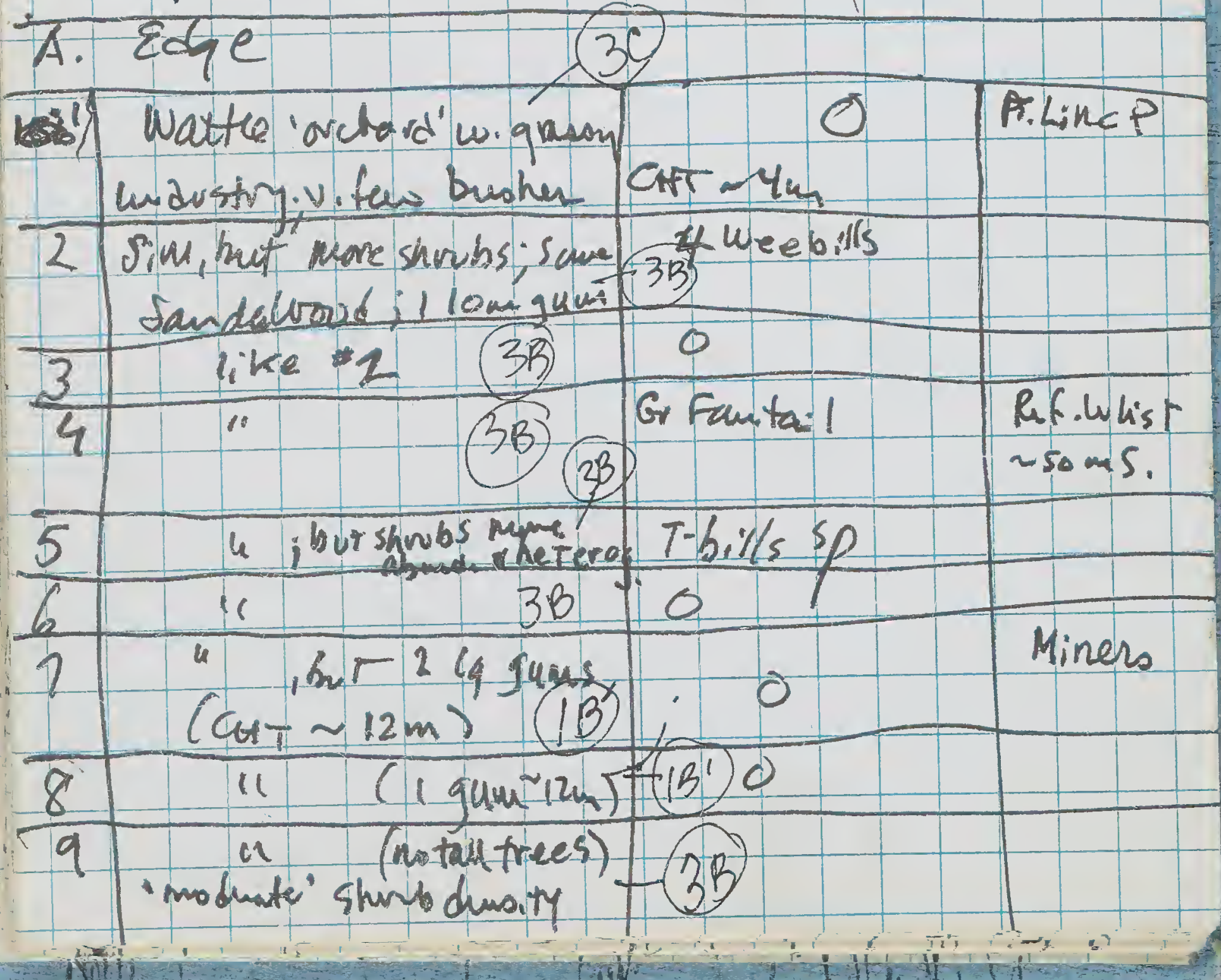


12 open gum wrode. 1 (IB) 4s. shrubs as alove (Bobloy!)

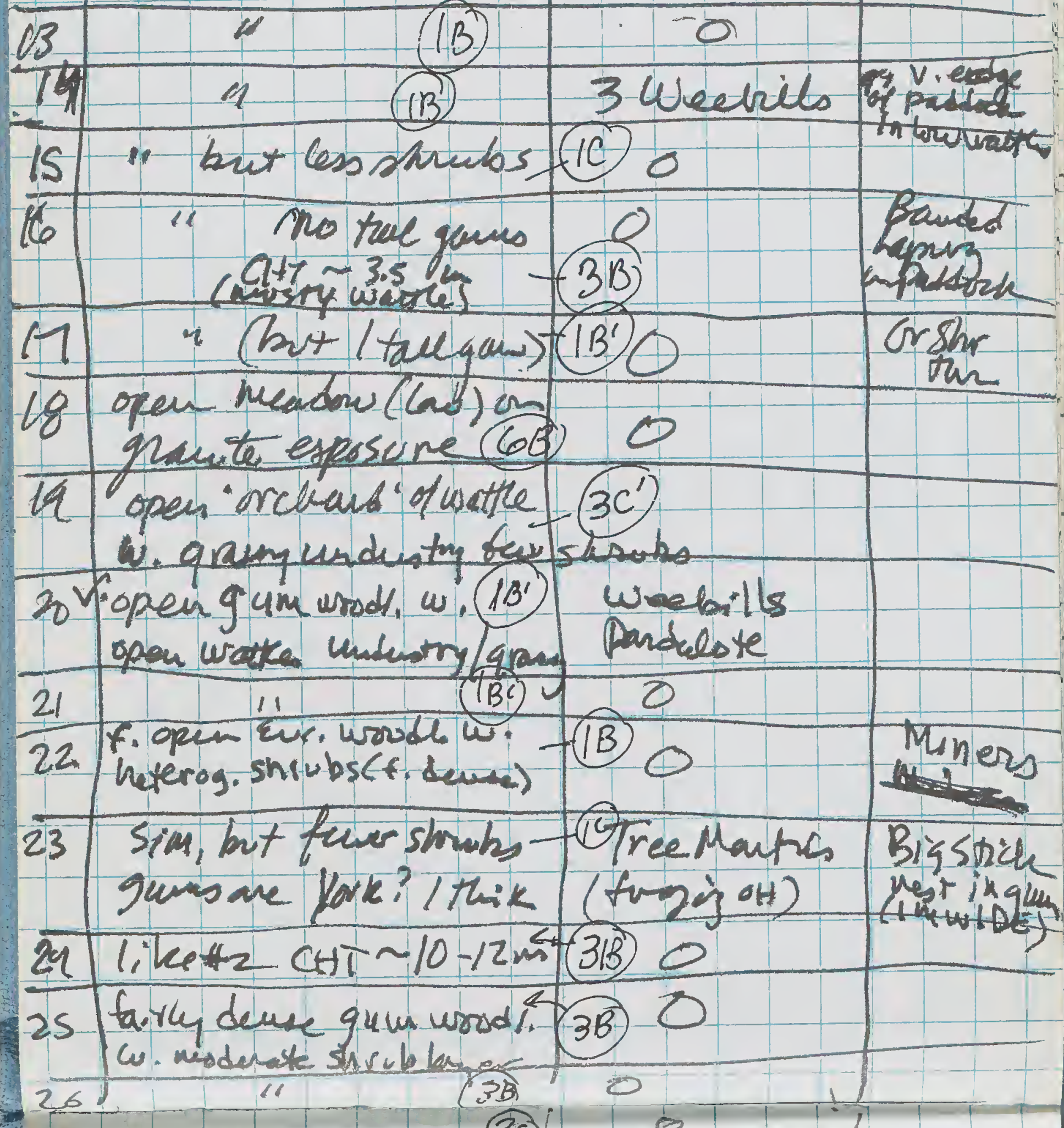

27 mereopen hut s, 36

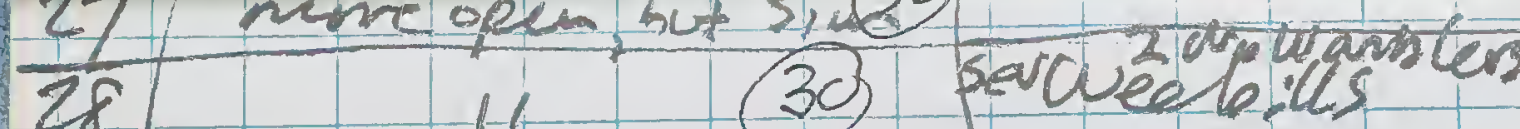

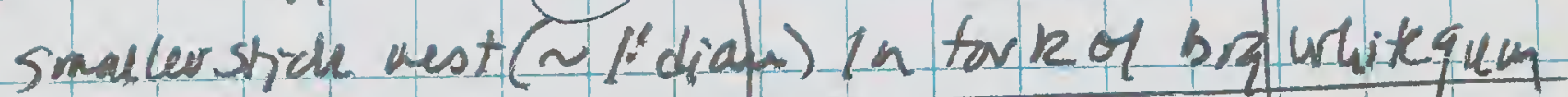

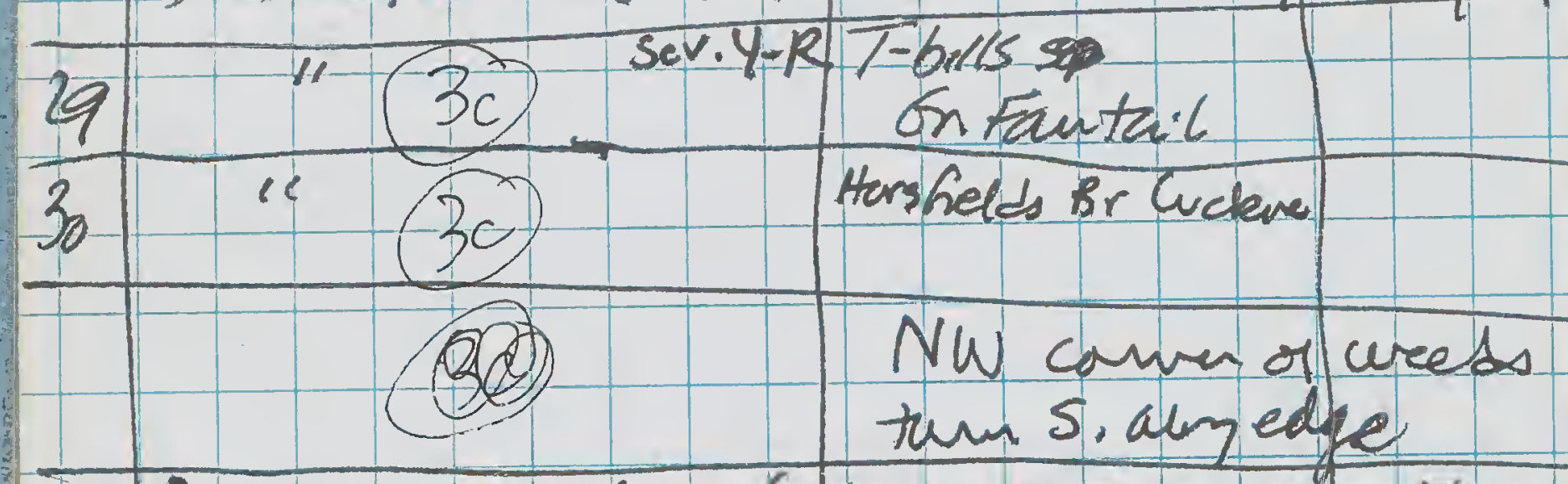

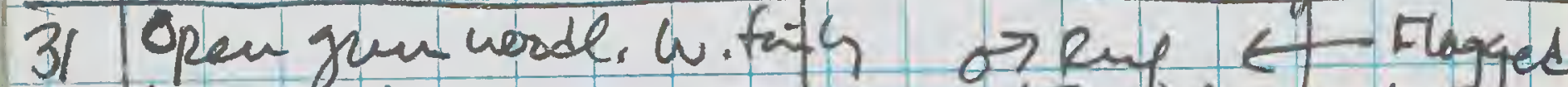
duseundusting of wattle wrister on Fence to - 3m-1 grases (B) Dif Z (P) fad he 32 7 4 Pr.linc Parr Pिद्युओ

33 ipen York quim to 7un (A)

wen arshrtar

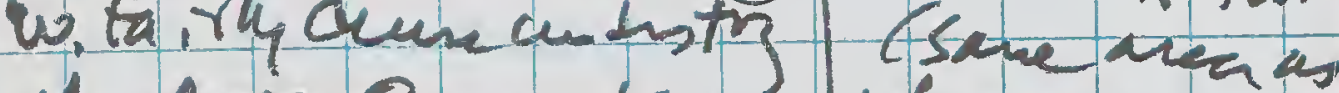
of Bacek.s (found we lat stor)

34 orchaid-type low werle of Jaw 1 Casualiva A. BB scatued duest the chits of shubs $2.5 \mathrm{~s}$

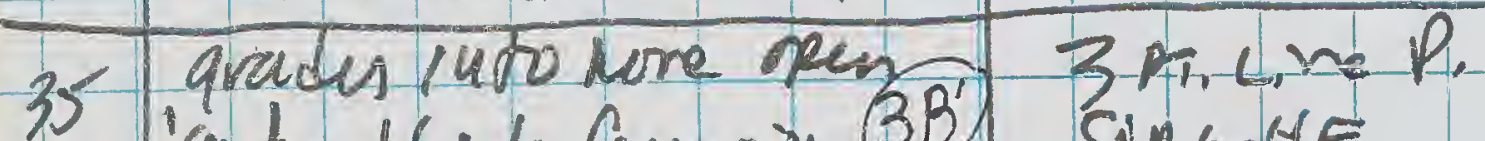
'archad' wo Gouariun (3B) SInG HE thichets GHT $3 \mathrm{~m}$

31 oper 'orchand' - watte 6 saudaluood. lorv, open avass-solye undiw $3 c^{\circ}$ $\checkmark$ scaptered busheo (Rhinglow) tock - fro aving in resmer. onden them in (and $\left(c^{-}\right)$fron hese 
open low orch' of watte aut ountaloond w. qrass-setge 4.5 .

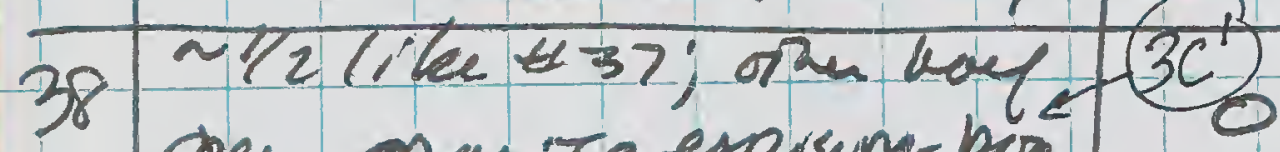

qeen graute exposura-boy

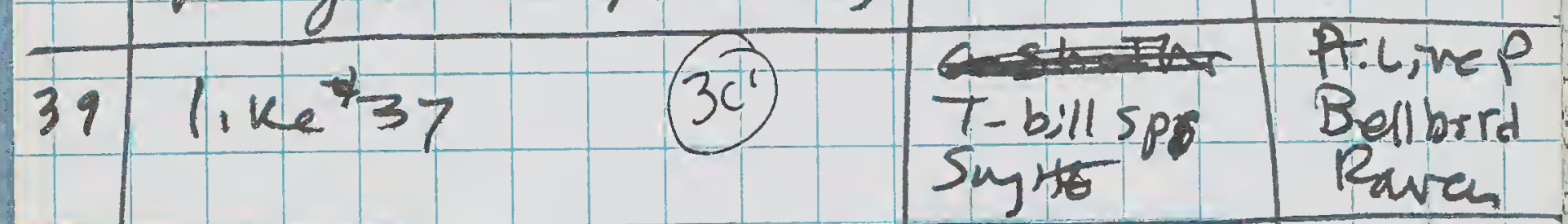

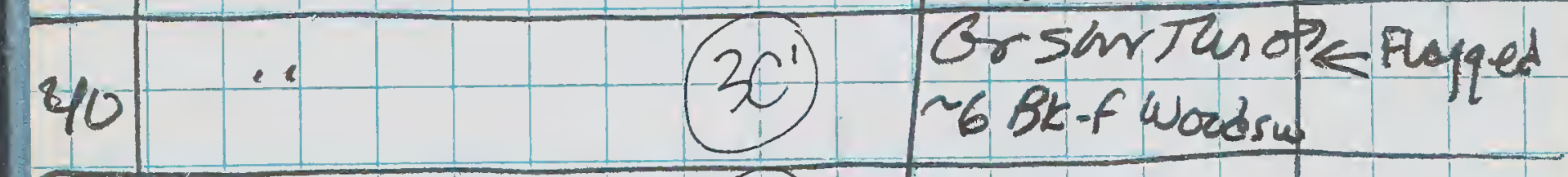

\begin{tabular}{l|l|l|l|l}
\hline 91 & 4 & $30^{\prime}$ & weebills (sev) & \\
\hline 92 & & $3 c^{\prime}$ & 0 & Juve.
\end{tabular}

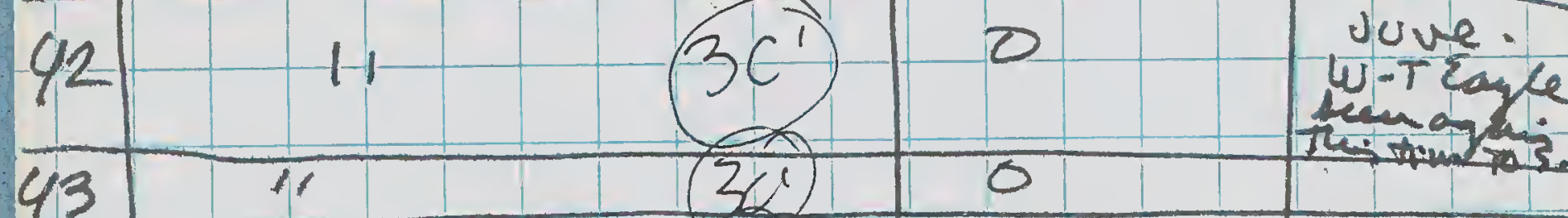

\begin{tabular}{|c|c|c|c|c|}
\hline 44 & 1 & $2 i$ & $\sim 4$ wordsw. & \\
\hline 45 & 11 & 34 & 0 & Ravens \\
\hline 46 & 11 & $3 c^{\prime}$ & 0 & \\
\hline 47 & 11 & 0 &
\end{tabular}

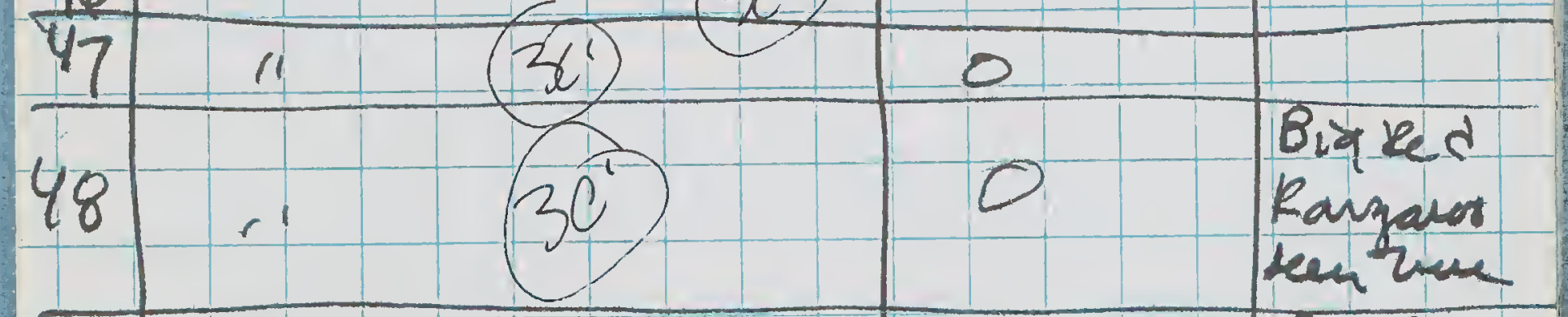

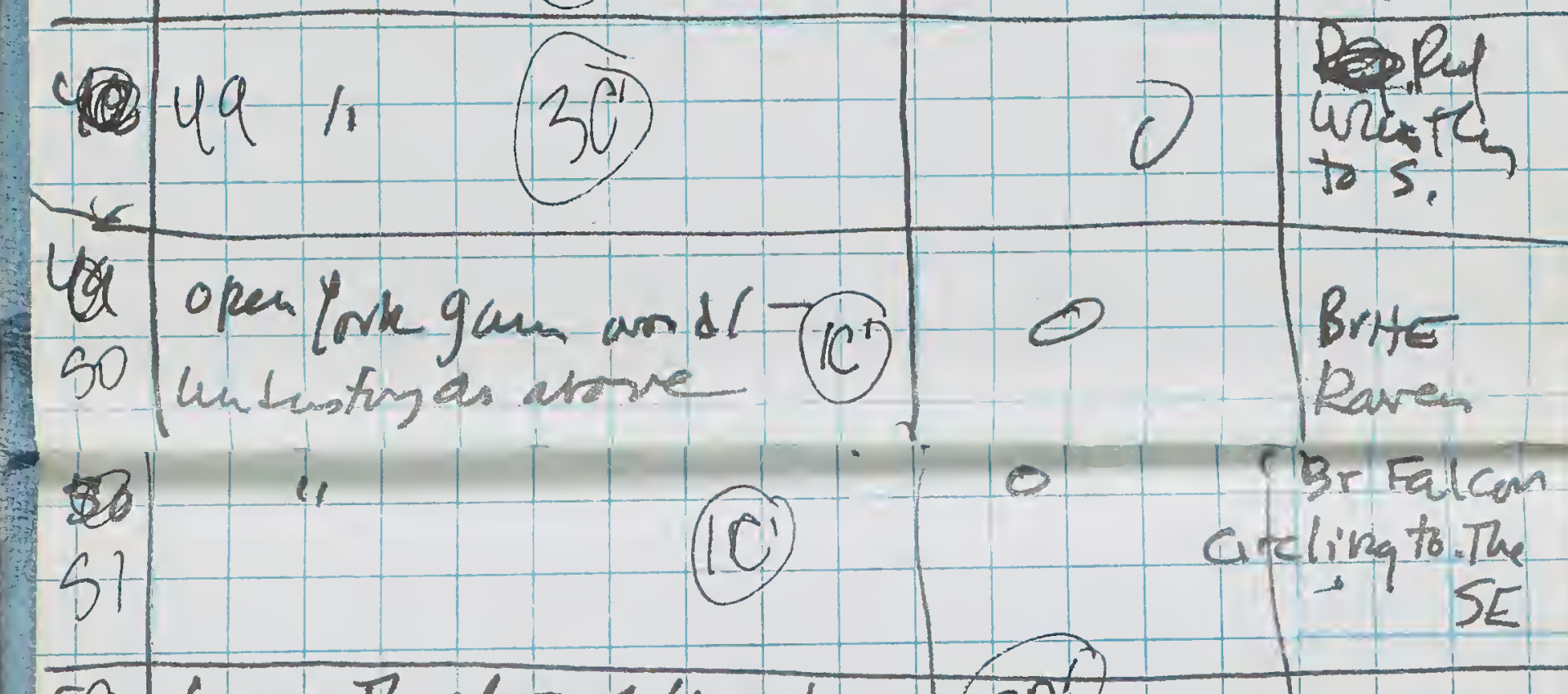

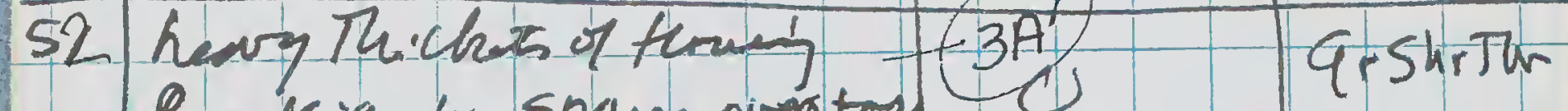
Baeckia w. spause overtoy C of wartle to $6-7 \mathrm{~m}$

S open quw und d w divese

S3 open quirisd w dived

\begin{tabular}{|c|c|c|c|}
\hline SBP & open 'orchand" waite & & \\
\hline & w. beturg sustend sunibd & Wechills & \\
\hline 540 & cst hay" " i ind "/2oped & & \\
\hline & york quin word I 78 & 2Ps linclars & \\
\hline & $(12)$ & $3 w, T \cdot$ bills & Prlunp \\
\hline & $(\| Q 1)$ & 0 & \\
\hline 20 & तिी (B) & & \\
\hline & "(IC) hut v-stry v. open, gu & mo & \\
\hline 56 & $\begin{array}{l}\text { Waith-sandalevood"orch." } \\
\text { opeu, grasy v-stry }\end{array}$ & & $\begin{array}{l}\text { Gr shrth } \\
\text { to NW }\end{array}$ \\
\hline 01 & 0 viopen (D) & 0 & $\sim 100 \mathrm{~m}$ \\
\hline
\end{tabular}

4) deuser "wch" - hut sim. (3c)

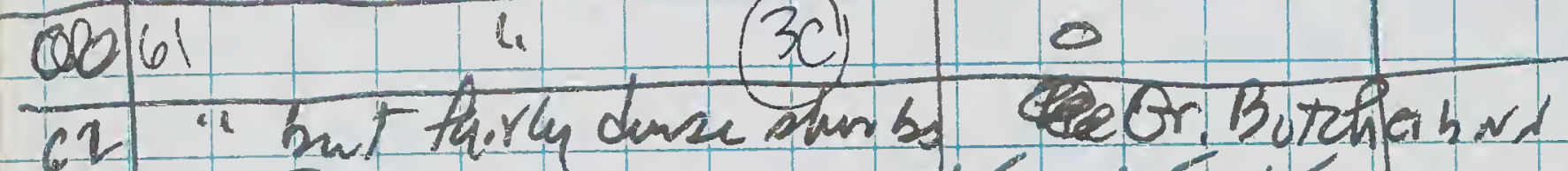
(3B) Cuil erated chée-chée-ché-enor-ch 12 oph low 'orch' w. Ga (3C) also a sweet yodelling is 63 granife expocure in widle (aj 'rol' a.divese shous byer W CAT $23 a$ (3B) 


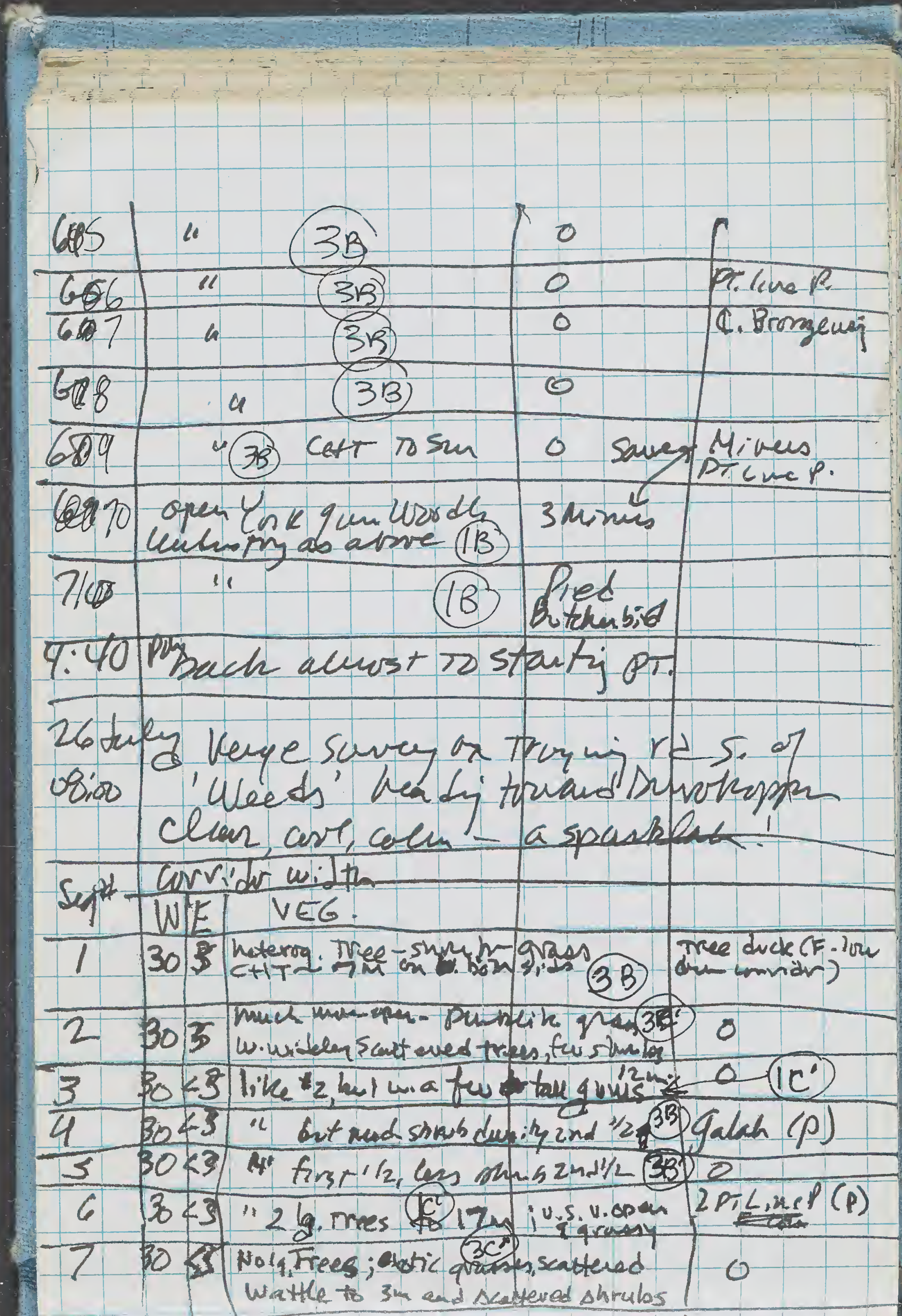

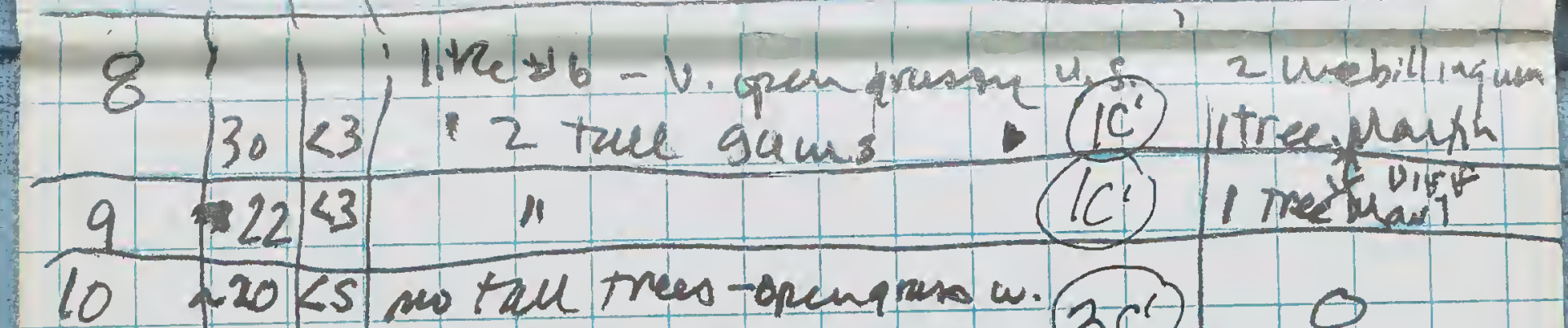

$10-20 \mathrm{ks}$ mo tall trees-openqmas w. 3C) 0

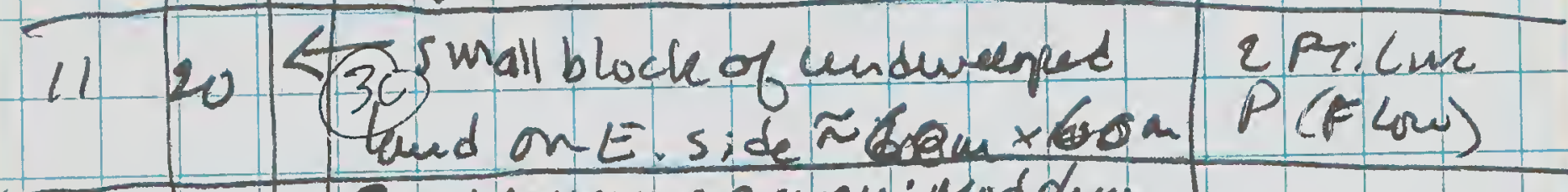

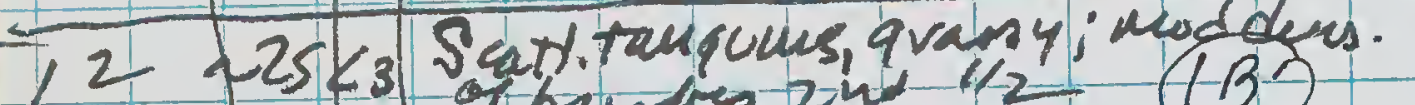

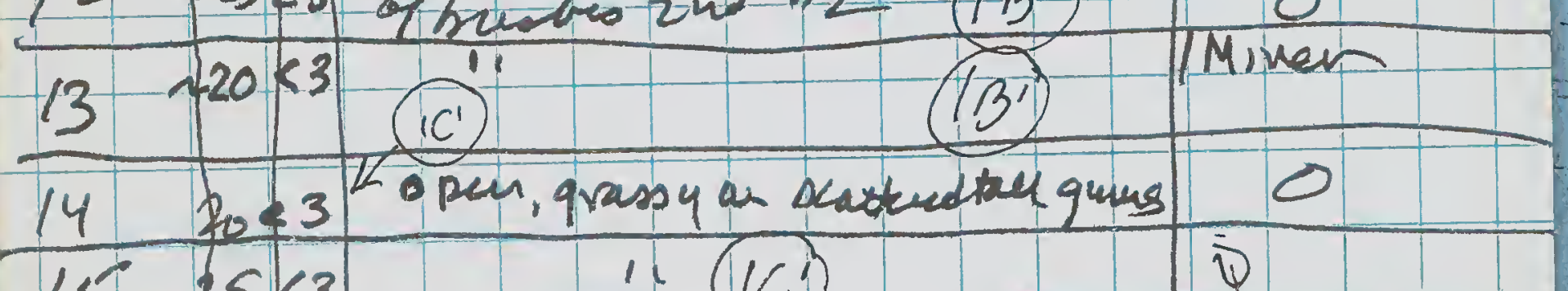
$1525<3$ il (IC) D

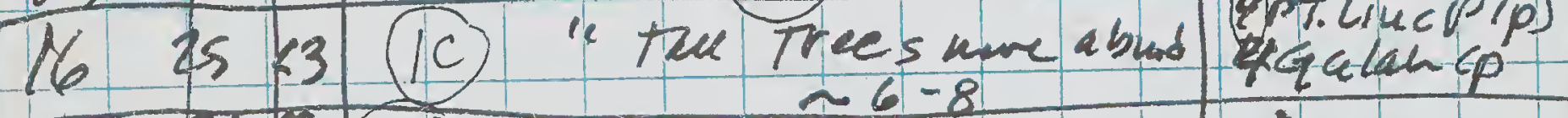
\begin{tabular}{ll|l|l}
17 & $26<3(10)$ & $\sim 6-8$ &
\end{tabular}

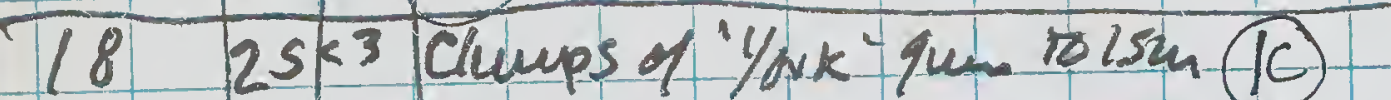
perkns 20-25 truaks in all 2 Magpies ( $p$ )

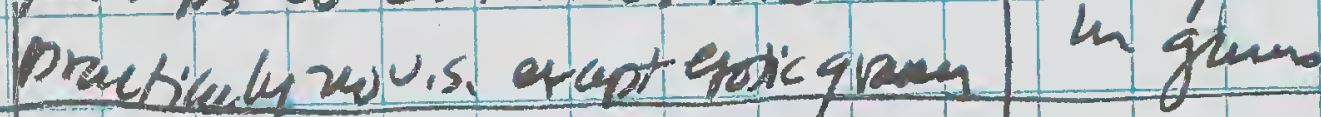

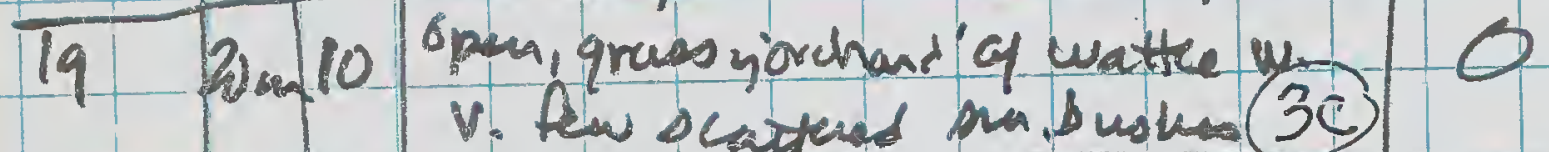

202010 "(3B) Calith duerbrushion E. S.6) 0 21205 " $1 B^{2}$ out abo $119.99 \mathrm{~m}(12 \mathrm{~m}) \quad 0$ $2230311 B^{\prime}-110$

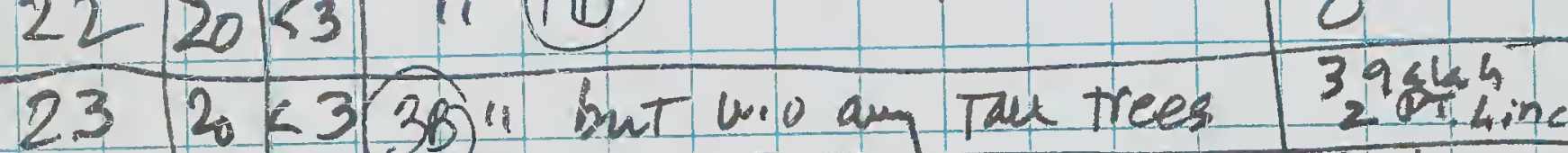
$2420<3 \quad 11$ w. I tae qum -3 T-bills

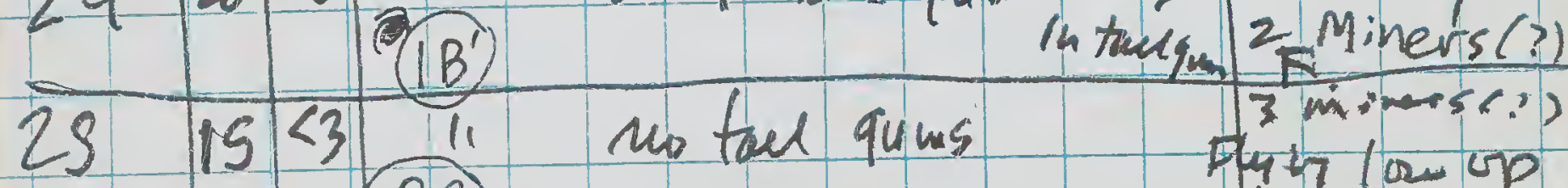
(36) the vas. 7 le is $\angle 3(3 B) 11$ Zqallah(ot) 
$+7$

27 ( Moukrakine rd. corts opphe)

2sk3 openiquan, watten Raven(ligh arf) shotued-in tall trees (3C)

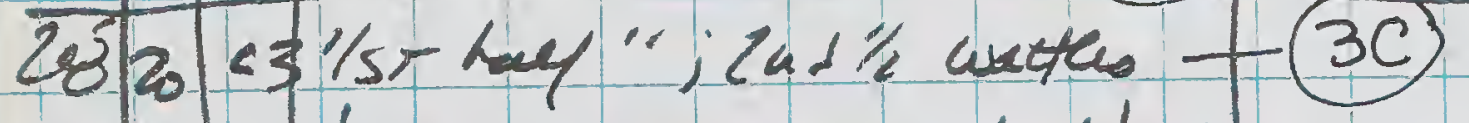
duser and sowe scatted hadp.

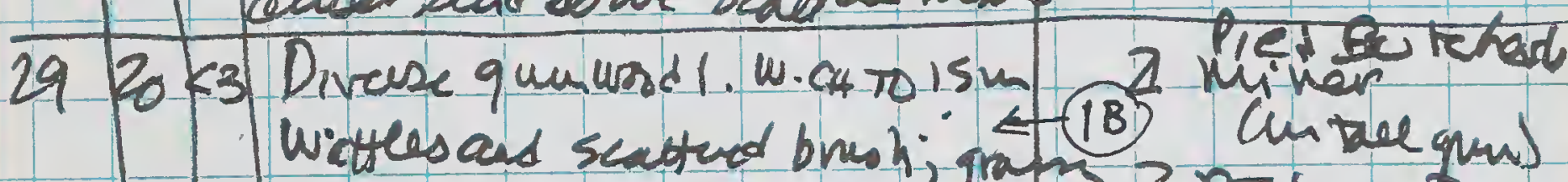

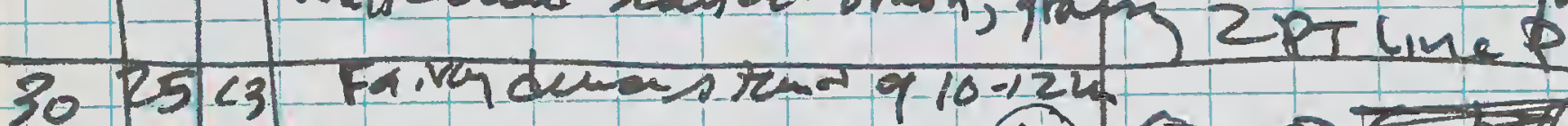

qumsw. oreu undurtung 10 sp

31 ask3 a bat mardshrubs (B) seathed though us (x) $/ 5 \mathrm{sm}$

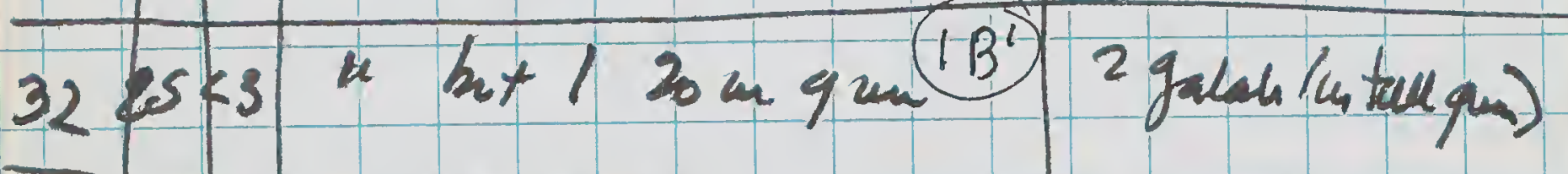

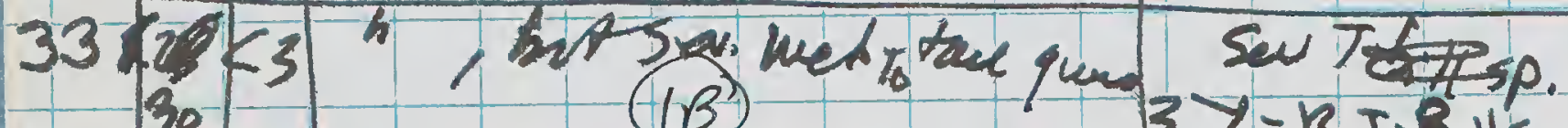

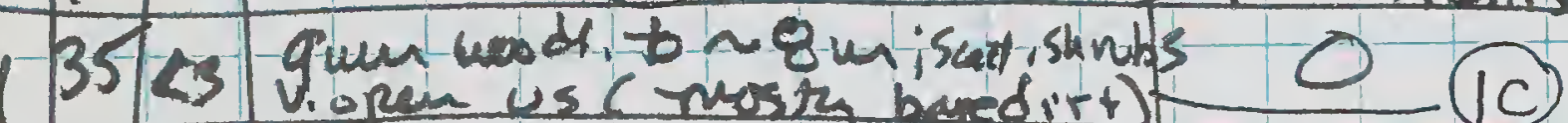

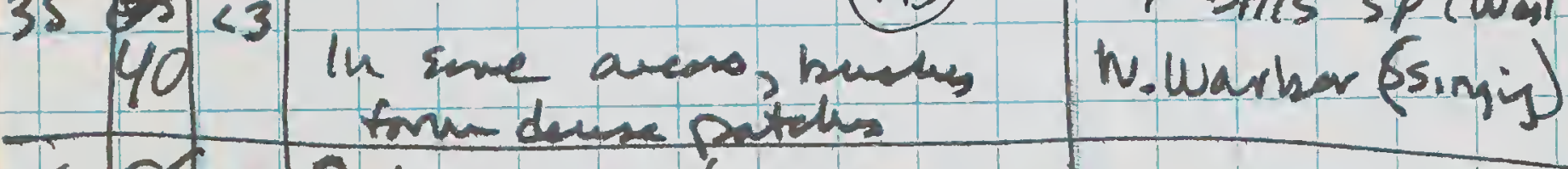

$3655 / 23$ fidme orch kallest treen hau

37 ploks 1 (3c) a minews 9:10 This prings un to caven to a sizeable patch here

9:5s Durokoppin East and walk border Punk on N-s access prade and

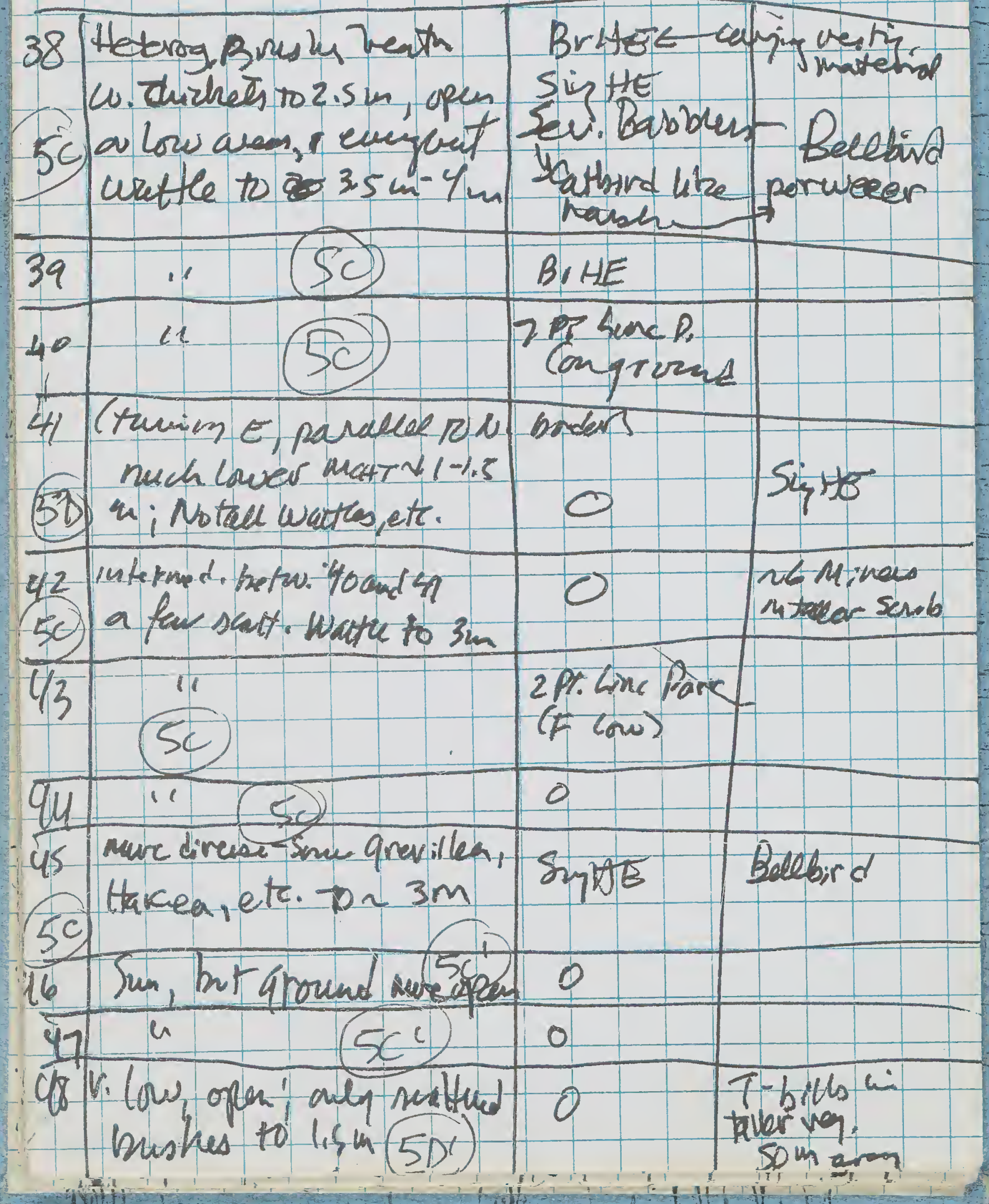


49 15t $2 / 3$ ditb; list it/3 a. mod. dens

5c0 of buske to $1.5-2 \mathrm{~m}$

So diveres shrest w. "ovchand 4 thenen

to $2.5 \mathrm{~m}$ (3B)

S1 Opew Hakew 'vdv' not (3E) O

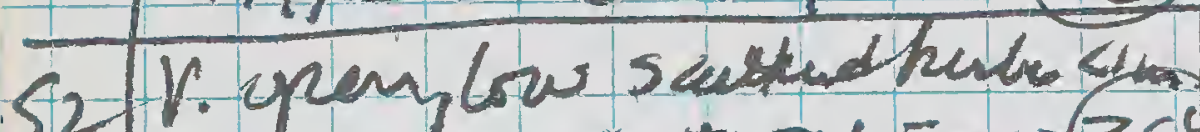

52 oce enugents to $\% 1,5$ or $2(30)$ ( 0

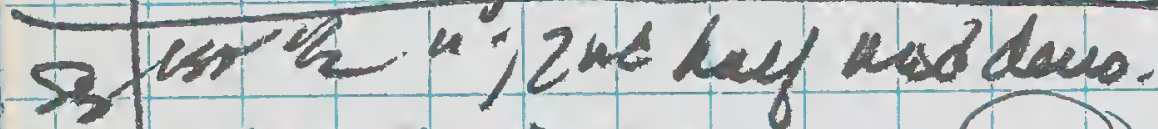

of sinubs to $2 \mathrm{~m}$

sq open irabue of Hatem ex

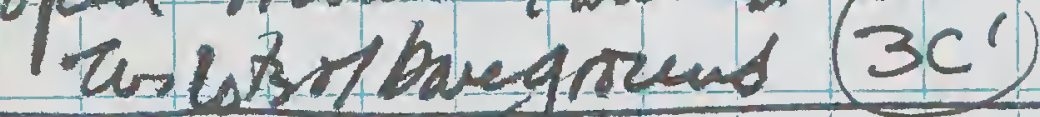

55 4

(3c)

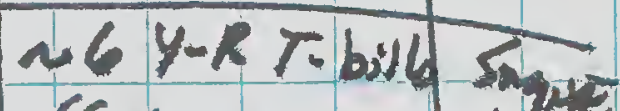
(Fetij ougroma)

56 deursthd of watte talea up to $4 \mathrm{~m}$ (3B

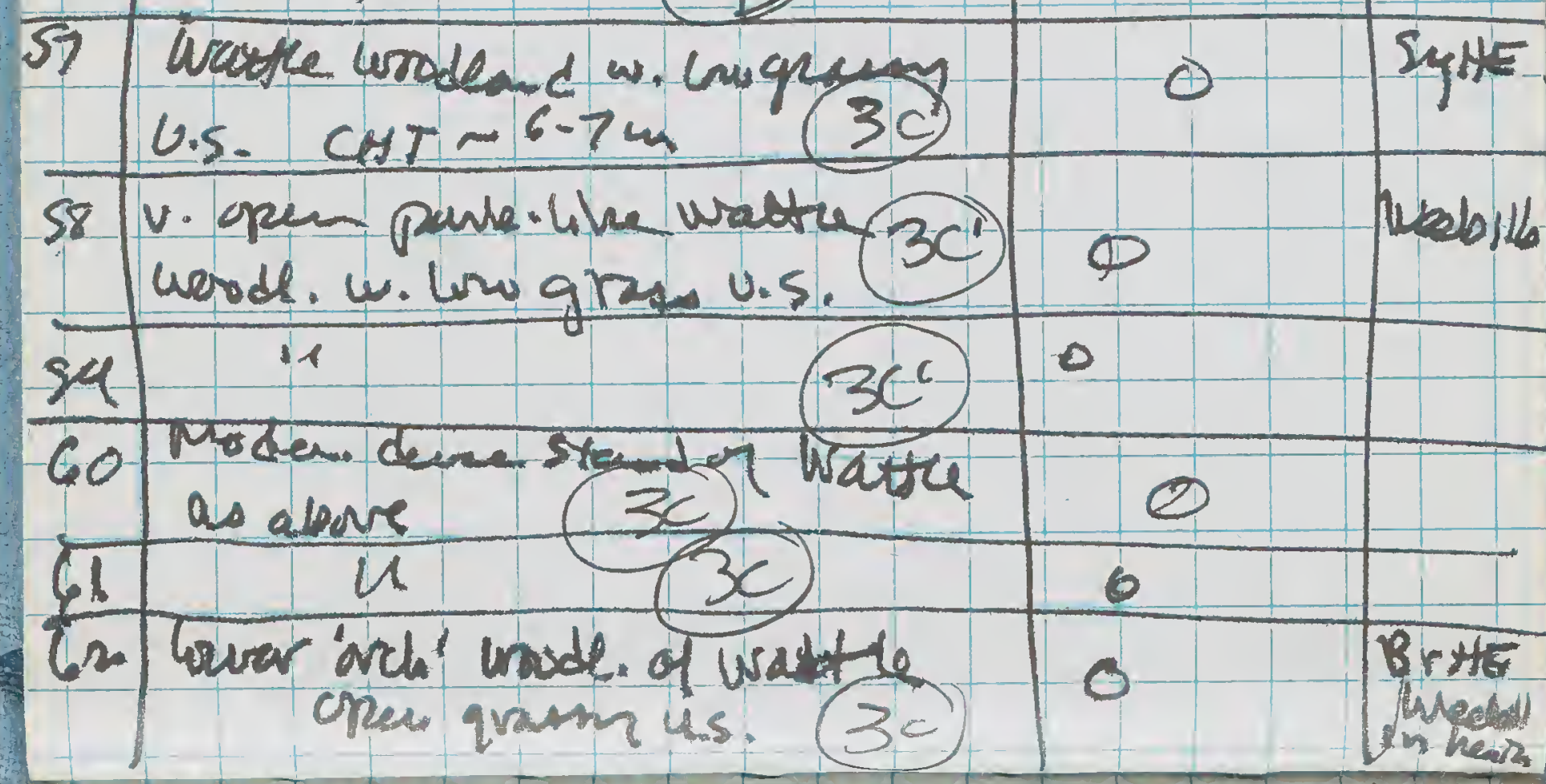

63 "(3c) Ete of waudor watl. 0 64 V. upen tau Wandos crad. (ICI) scattued lun shrubr, musta lougras, divt

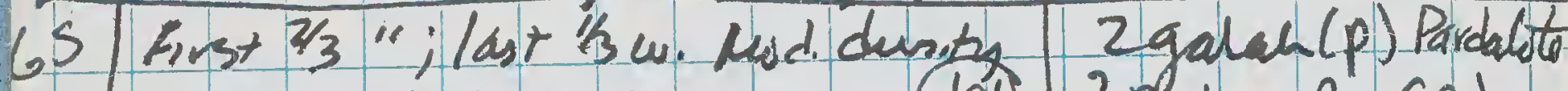
or saplip des oper kusus to $3 \mathrm{~m}$ (IC)

2 M.Lic $(P)$ 2 ps me P CPJ ow. Weebillo

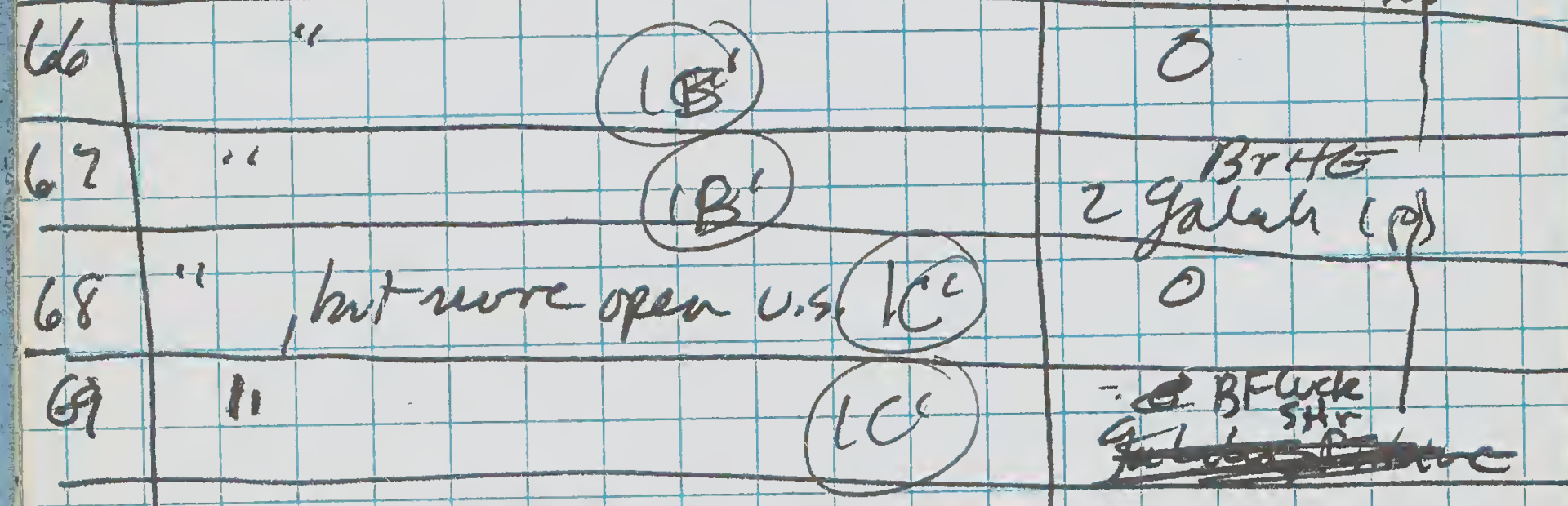
Nru move to reredsid SEgGite VERGE or JET Missent tham 2Pr. (inCPLP)

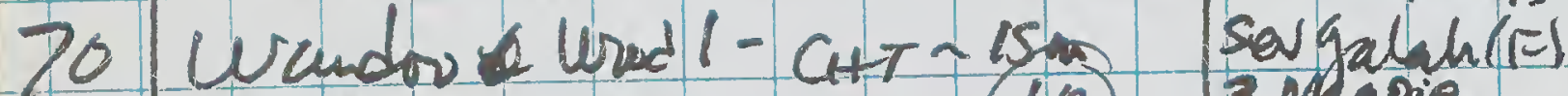
watte U.s. (IB) 3 Magpie

21

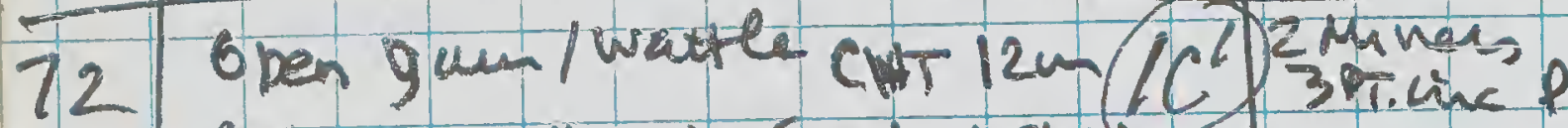

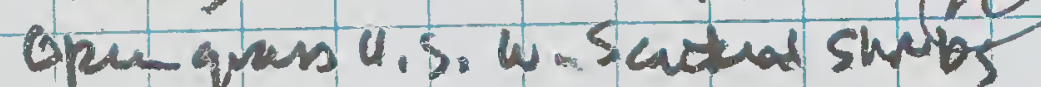
To $2.5 \mathrm{~m} \sum \approx 50$ tom 15 bed" i zuch bay quate into gesun 'orchan.' of takia, watere 9r. Puther 2 cri pinem 7 koth STRRT Ni un Missión RD ceom S. Sides 74 u upen parkline - a ferw ( $a$ ) T-bill sp. Acattered la quin ton coes grany US widuur t ho bushe 3 mivers
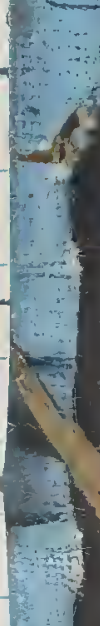


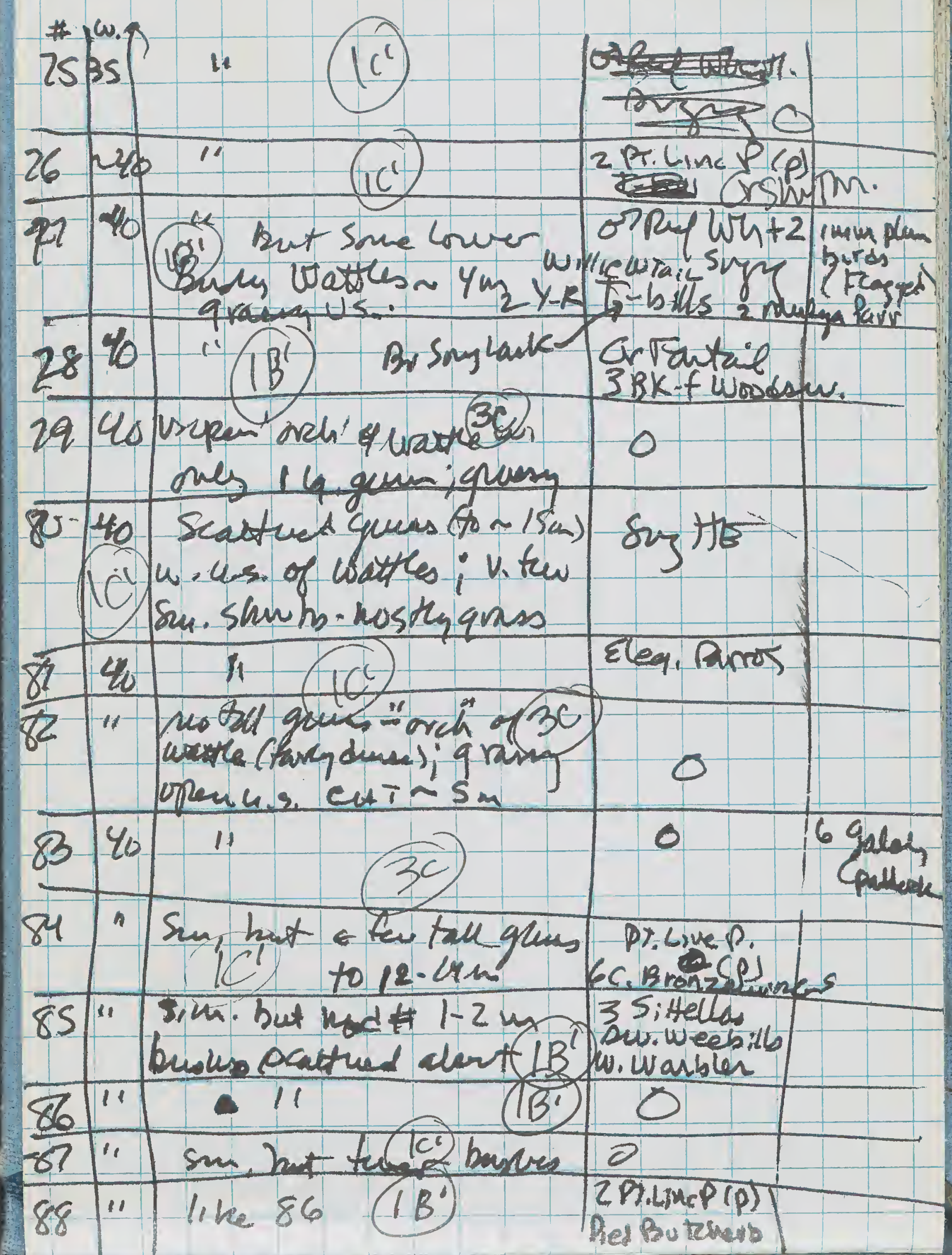

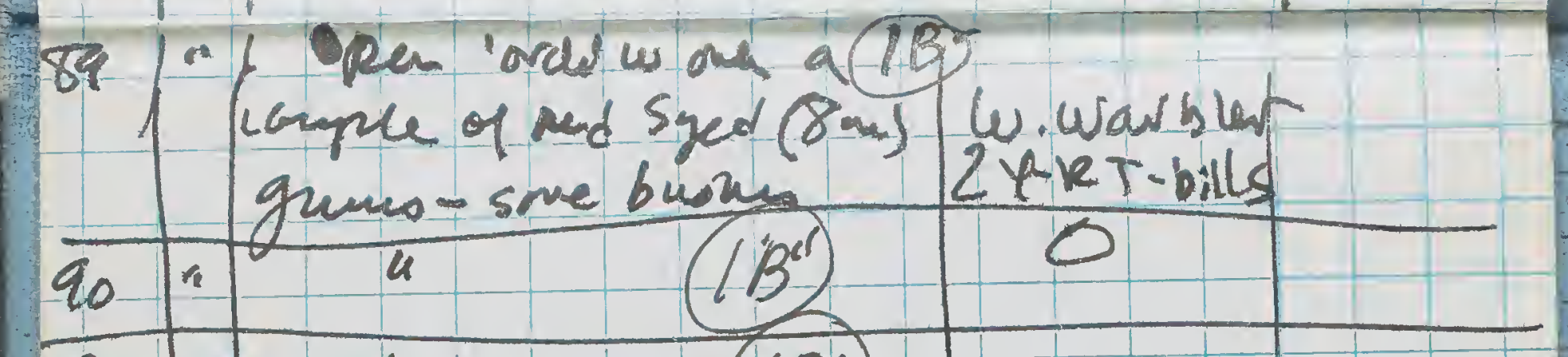

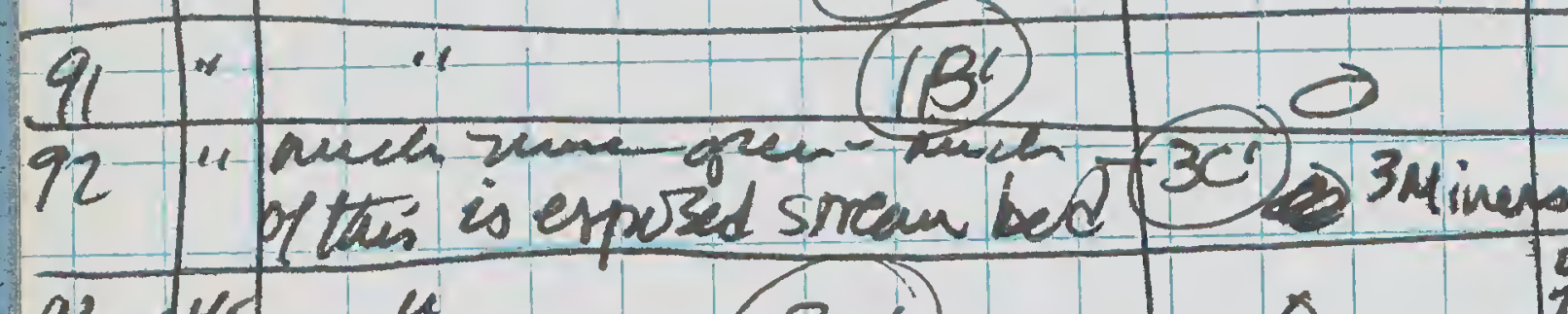

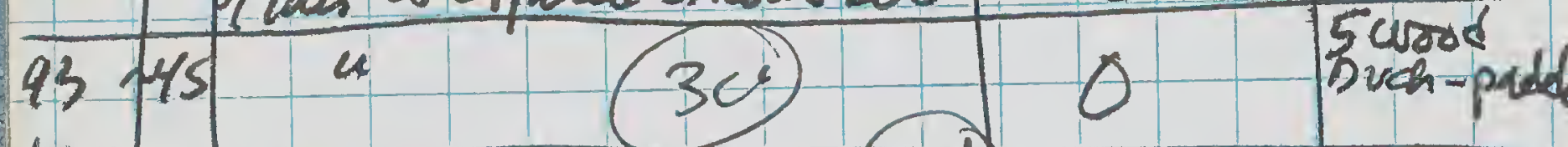

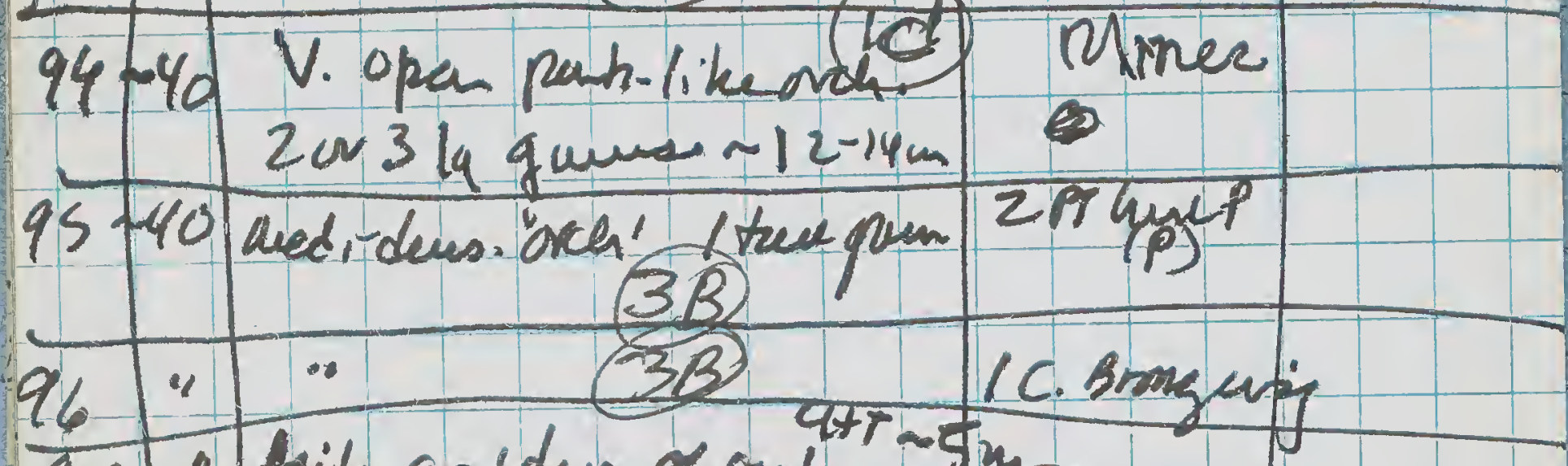

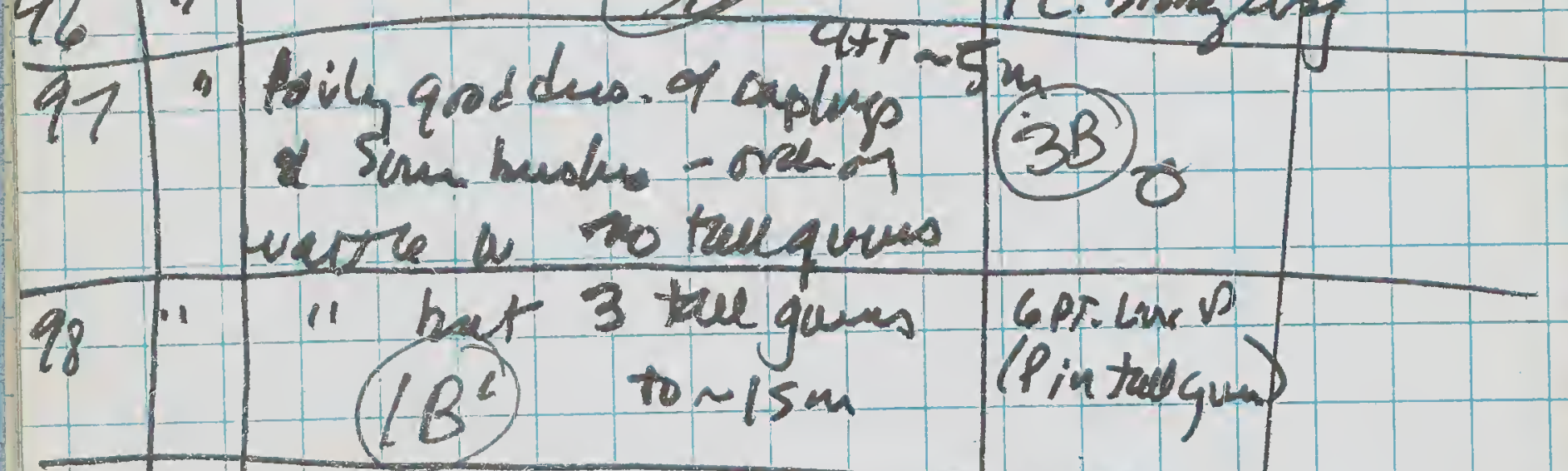

11:35 sioplereat entrane io largefaru on E. Side (save one) Aw Prom the otenside a foudpage) Nanpe cangin vest wat. 


\section{6 dulut cont'd}

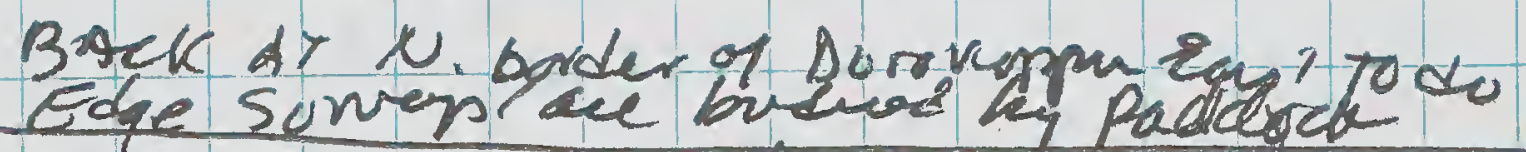

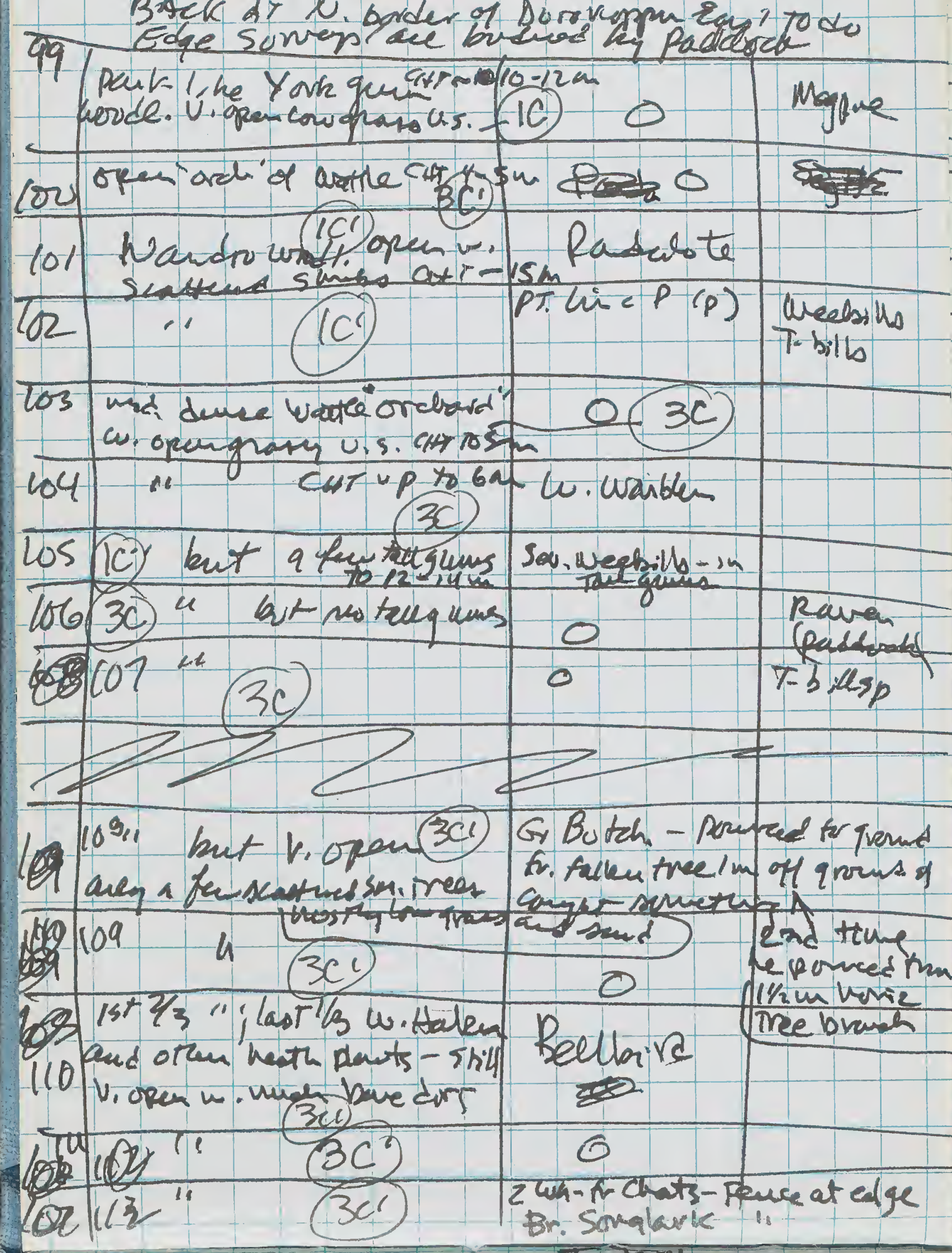

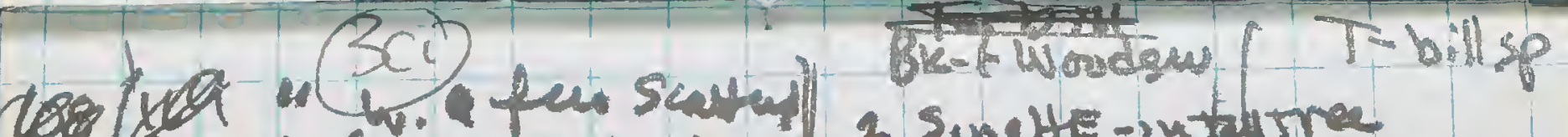
wh wate (Cit - 7-8m) $(13)$

60905 " 30 "

\section{the}

Les $A_{1}$ (1) 301 2ane 2 Singlfe-intentree conefid on spictiterantion) 2 Zebra Finch nave, have preteled - nyan. nyea $Y-r$ T-bill-reet 1. 7 m up in a $\Rightarrow 2.5-m$ spinf

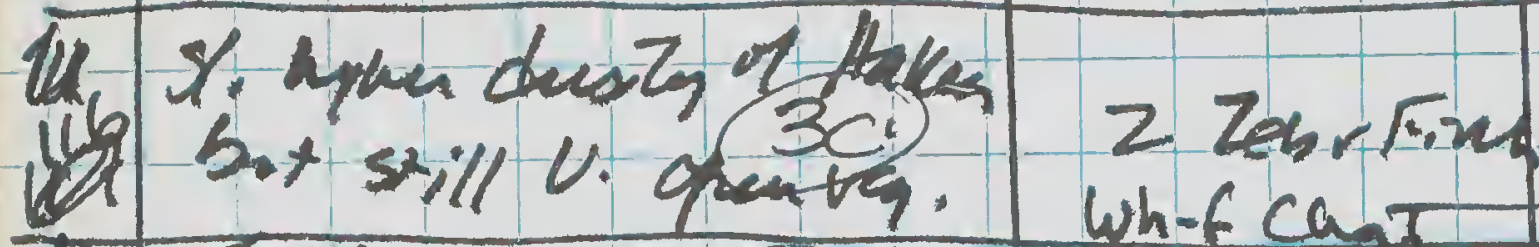

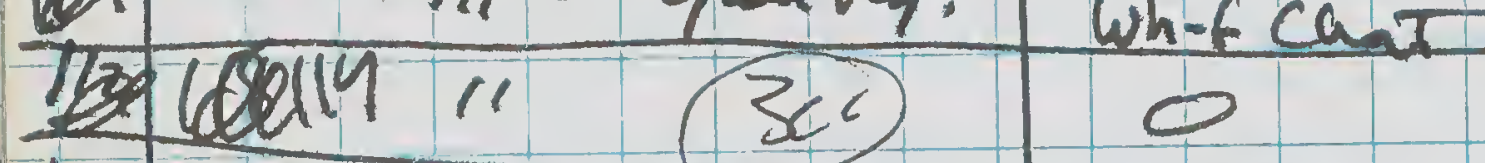
Uts diven shoub/haith/satige 0

H(100." So CitrTo6m Singtte T-bills 1010 (SO) o

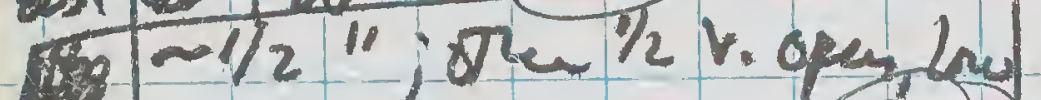
12a W.lobol baredirt (Sc)

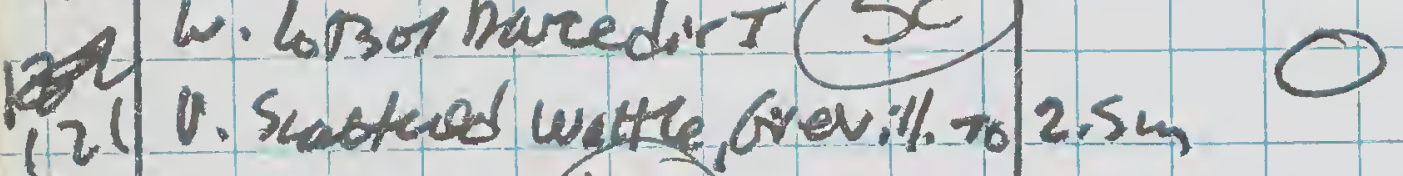
10 a sD 1 bire sp

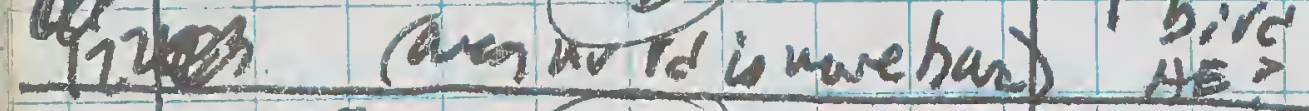

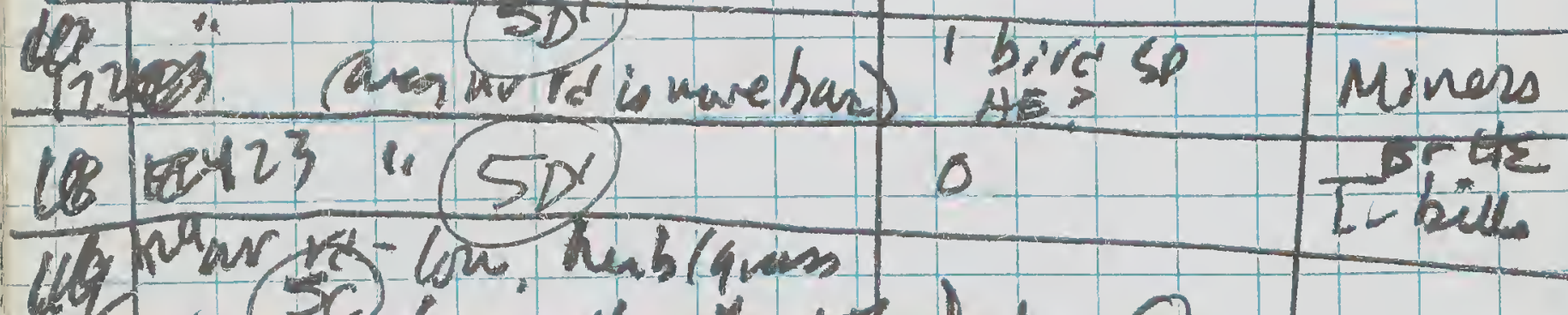

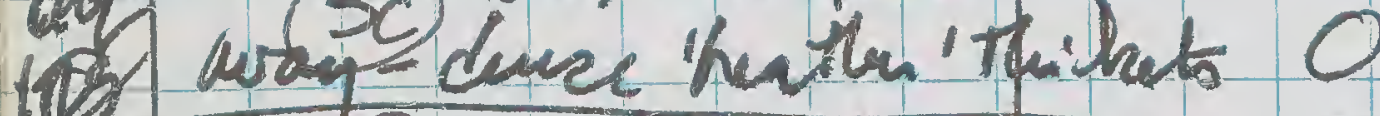

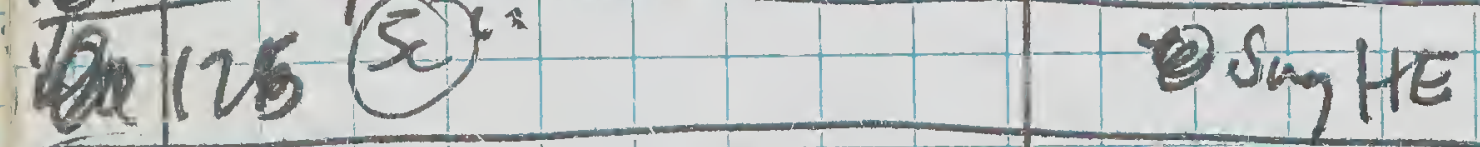
12:50 Entrave Re hue' Stop Nakeare of exit of track notekrou rd: -02.65

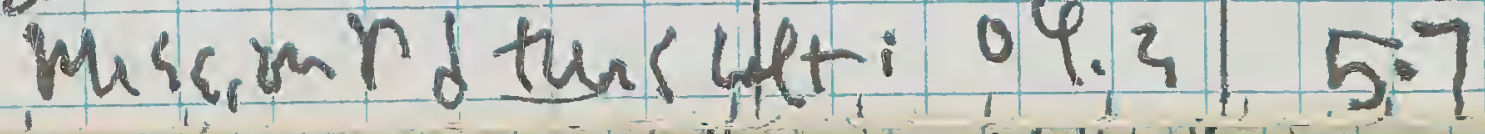


27 tuly. Raf. Whisten or sizin at

The very. NW awen of Durokipgin tast

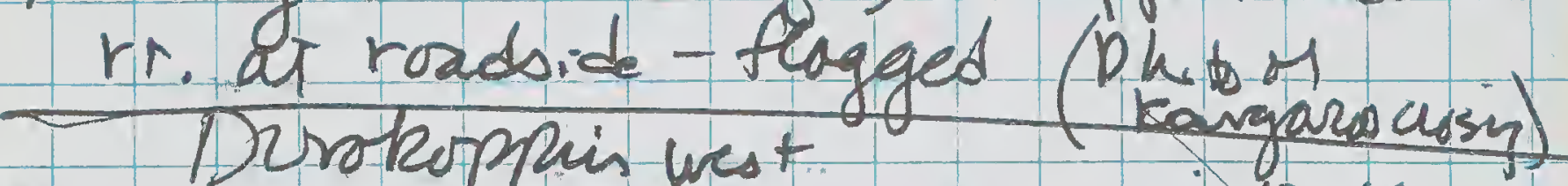

Ebe traust aln $N$ bordew w. Padderle

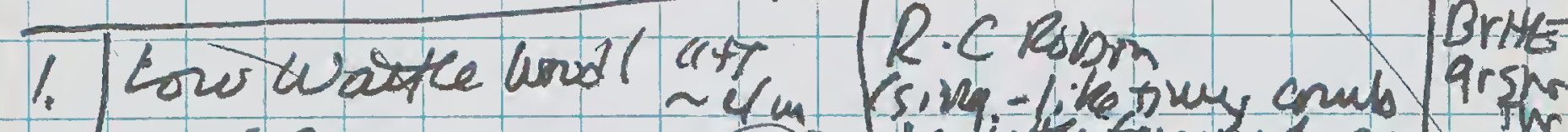

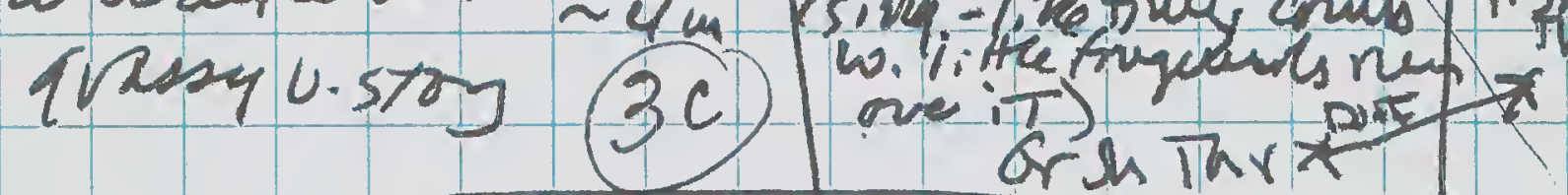

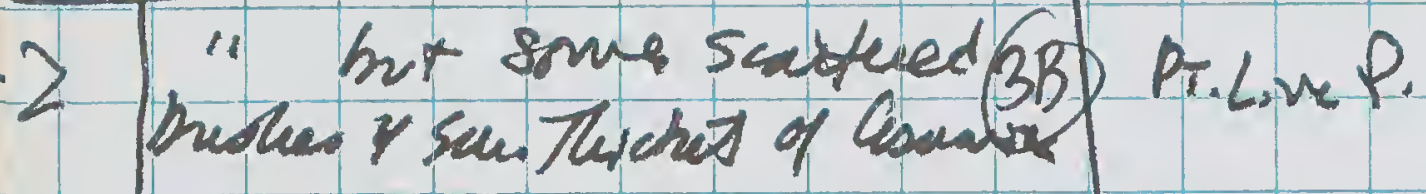

3 A bot soatiqueus to $12 \mathrm{~m} / 1 \mathrm{~B} / 2$ vacky $w$, farvly oreen questy vistong BrHE

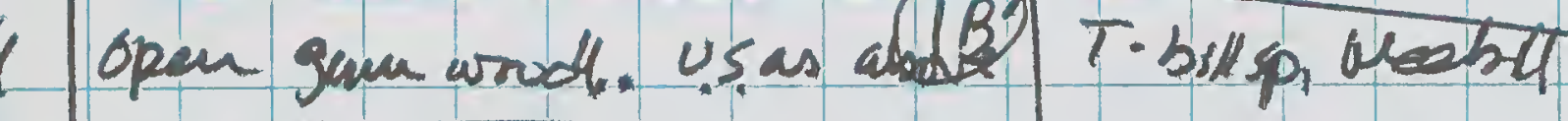

5. Alomy fence - g un wood, strip

inlaud-watte orch.w deve IB O

Cempo of Casuming scatuad

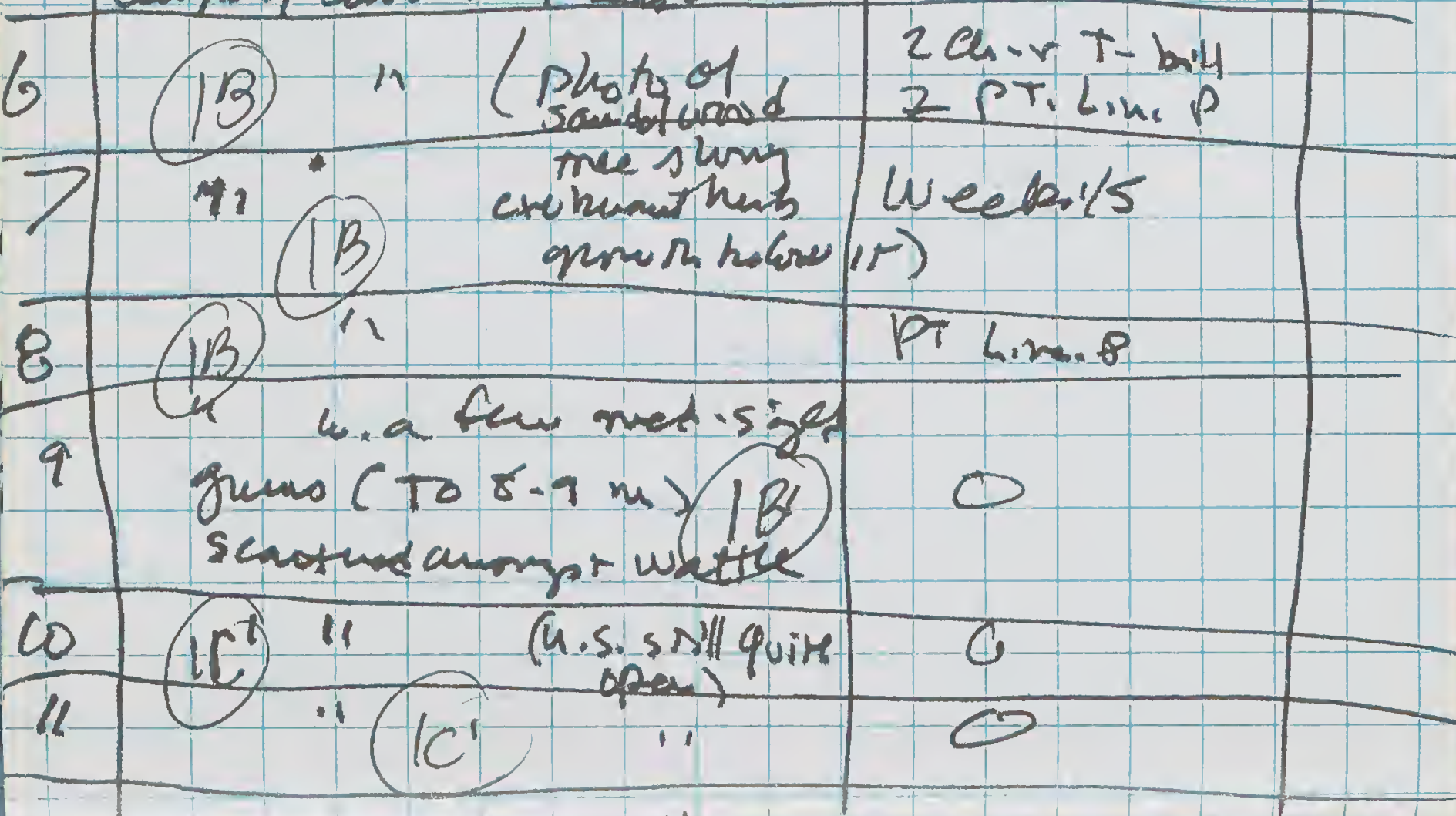

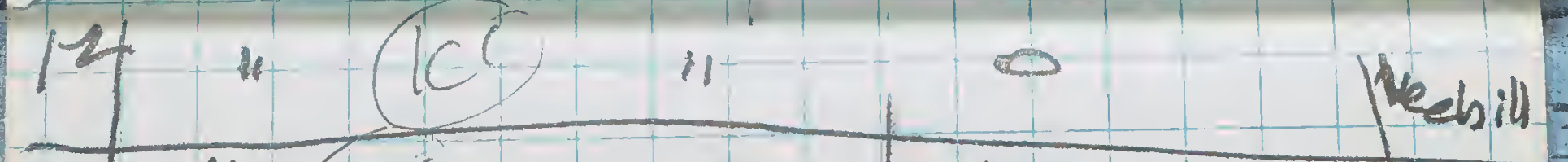

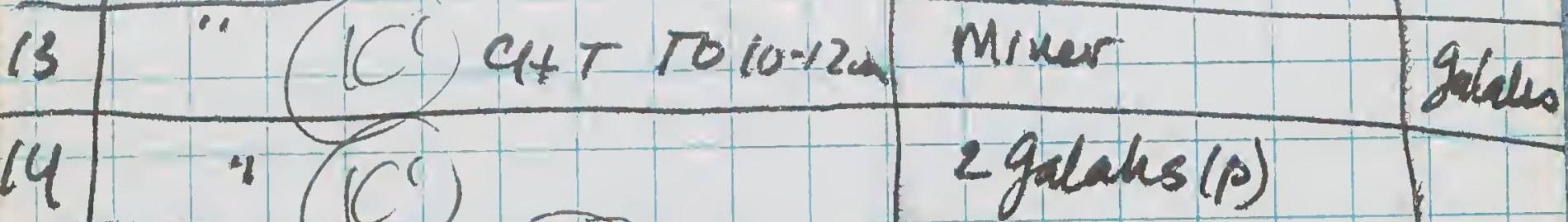

is $\because(1 \mathrm{Cc}$

16 qea wardool sweun $\left(1 c^{4}\right)$

wooth CAt ro zomil B. Lic $R$

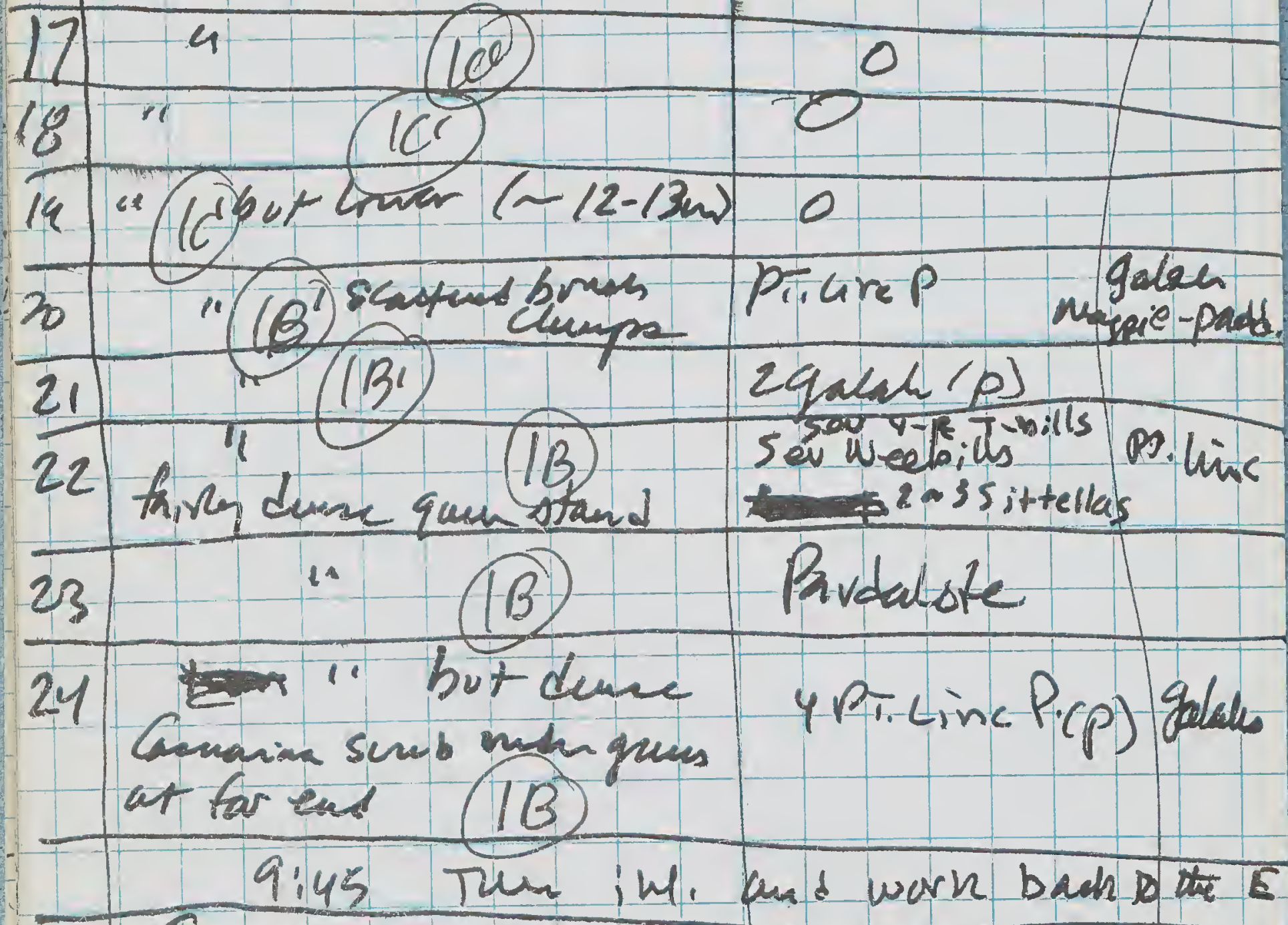

is TaU qui uondl. u. haw 2 galdh ( $P$

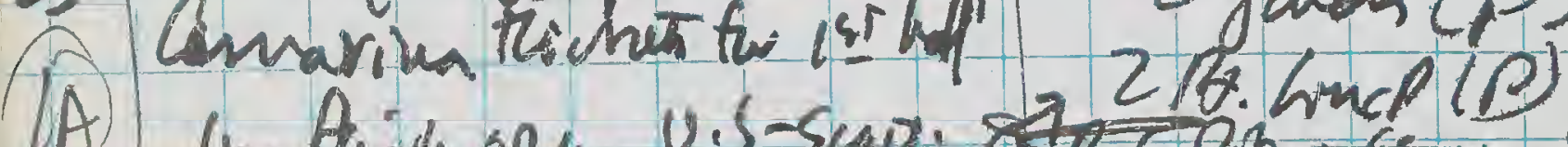

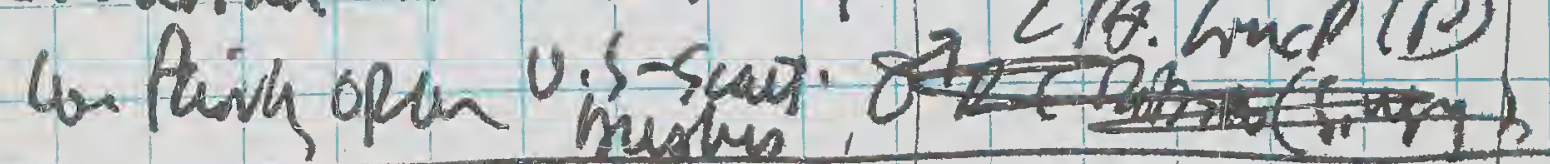

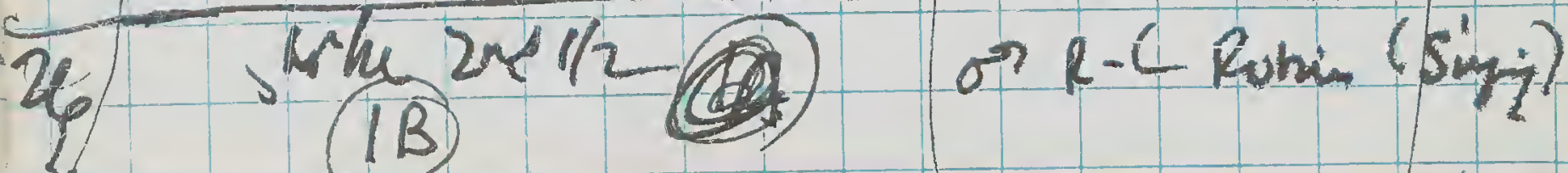




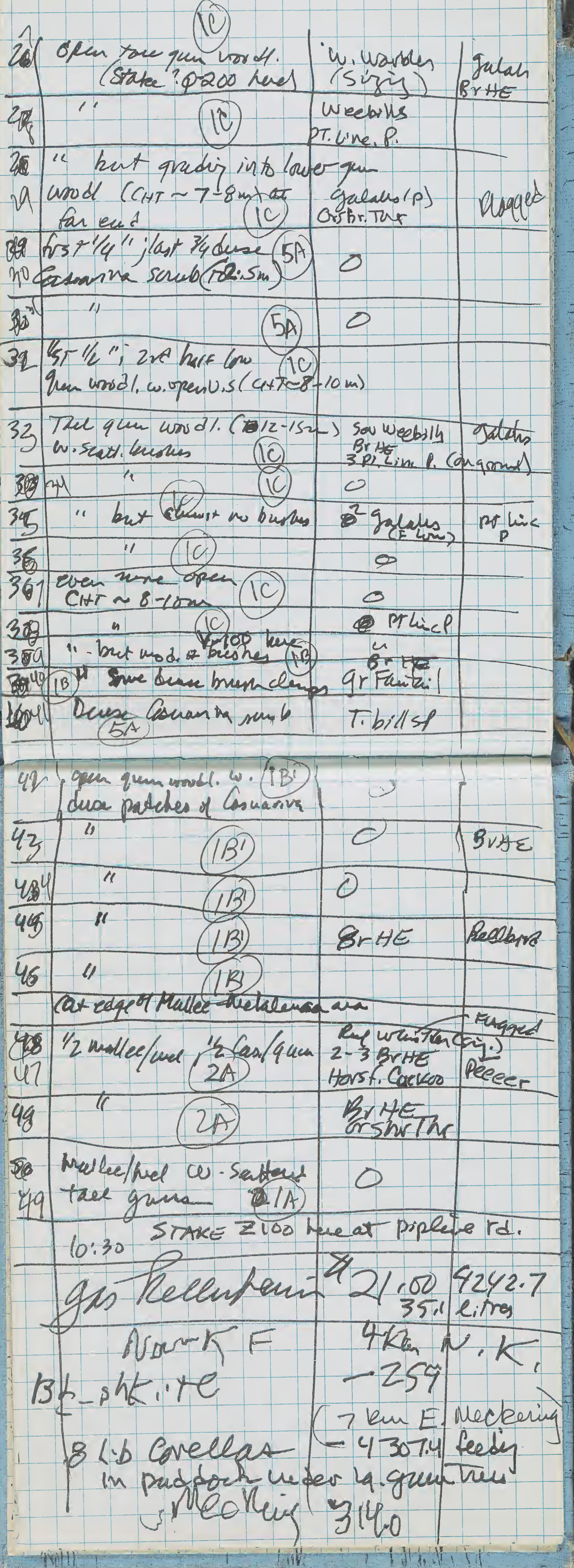




$$
\begin{aligned}
& \text { Kife - 314.0 } \\
& \text { Nowken K. } 357.8 \\
& \text { Kie } 341.5
\end{aligned}
$$

$12: 15$

Diortham Nogens of Mute Swans, Cov t3, Blach Duchs, Welceñe furecenus (huntivar watar) swauns praj up suchisolom fa vestin?

Retura Heleva Valleq 784,430.8

3OAVE 486.1 start

Gas 498.4 603.6

kite 673.8 Nanteesk 677.3

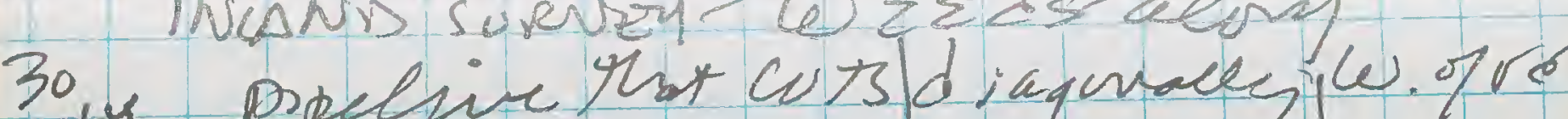
jukt 13:35-cem, mild, st breege

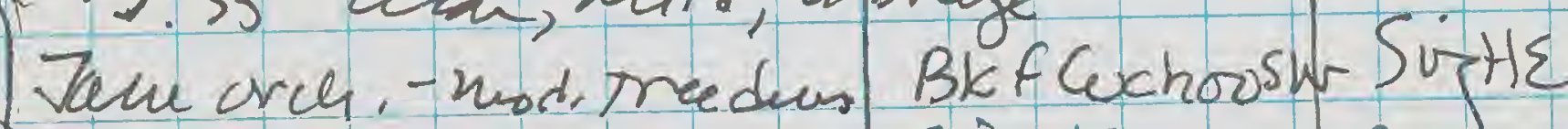
singte.

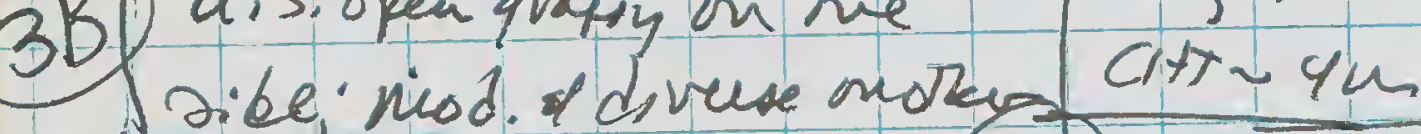
Bute Raven

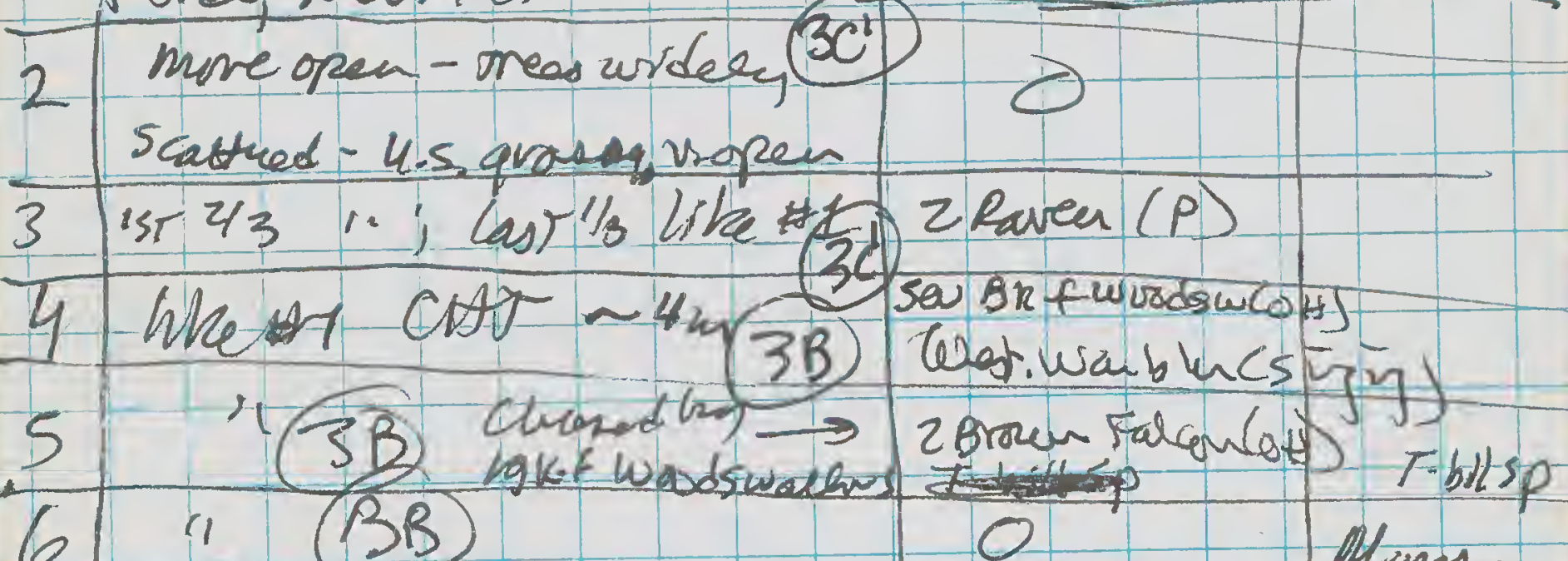

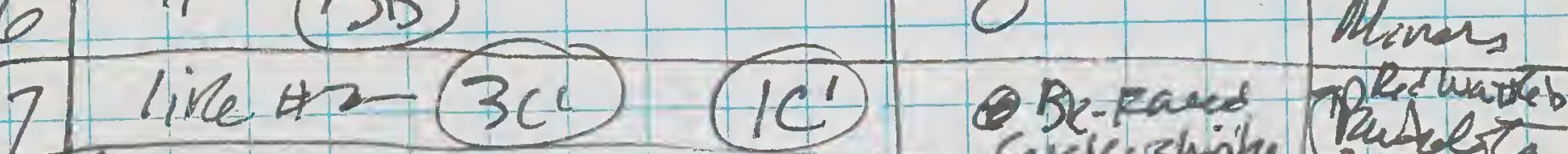

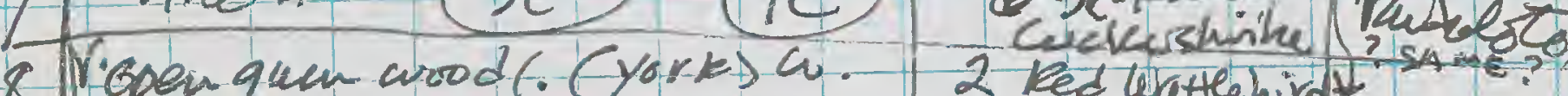
2 ked wattlebird V. Sutrued sumand grassin 0.8 . Kit5 $\sim 24$ 1 Hetervo. vam orchand w, tation, (3B) O GS4. R2

10 Iam Loond. Cat wpto $8 \mathrm{~m}$ (3r) Ereq Butch. w. farly devese shublagev, quesses Br He

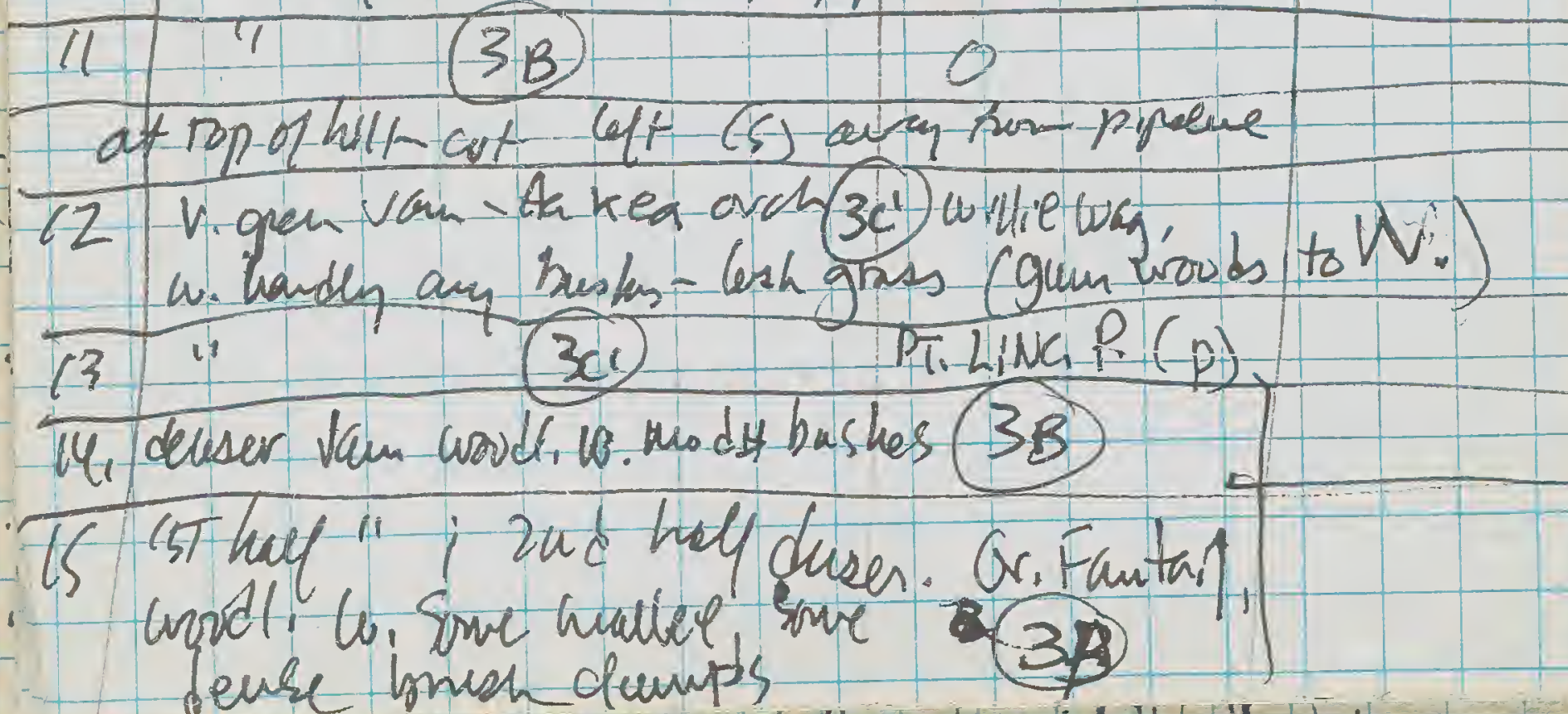
eure brish ofunps 


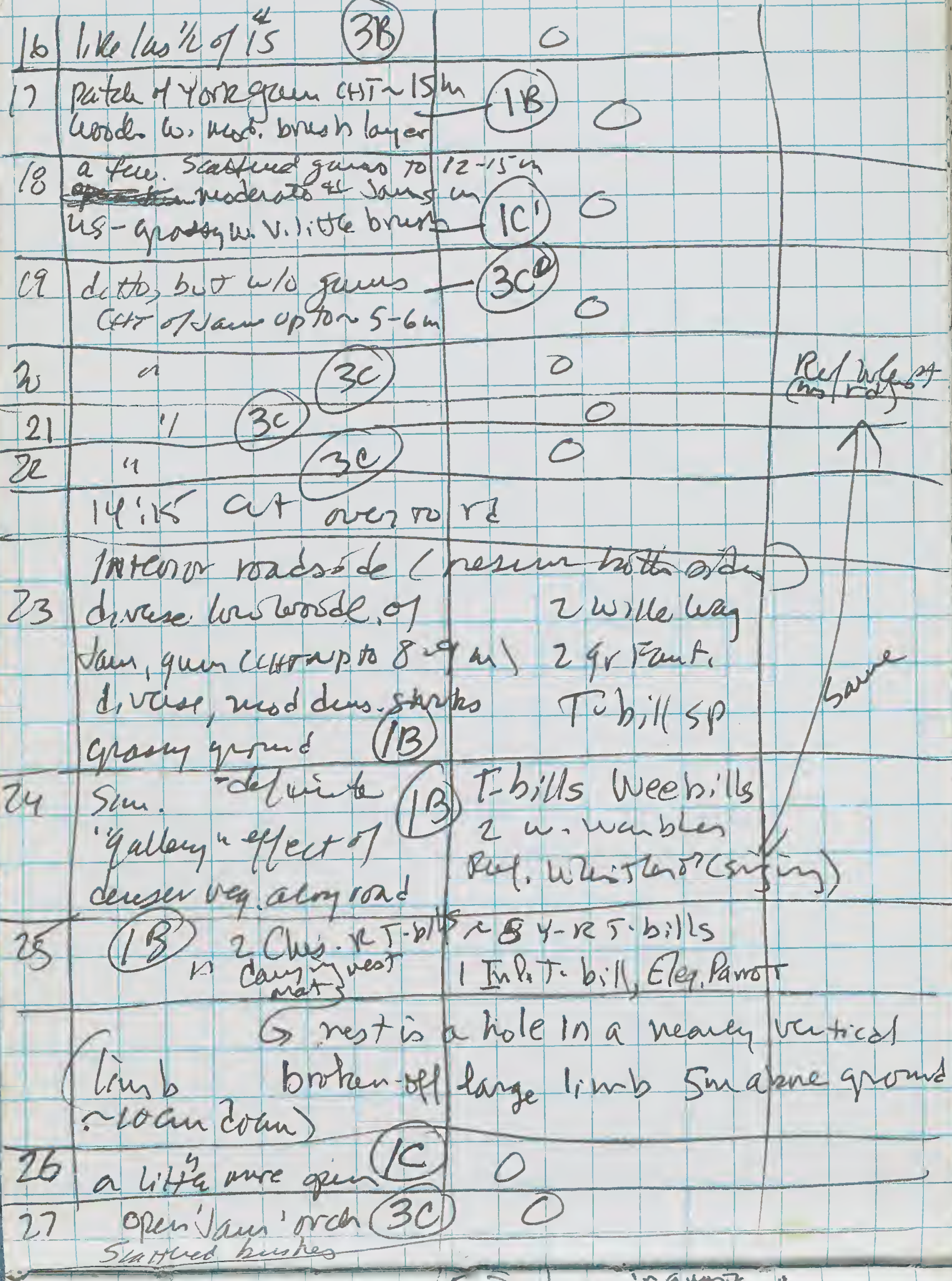

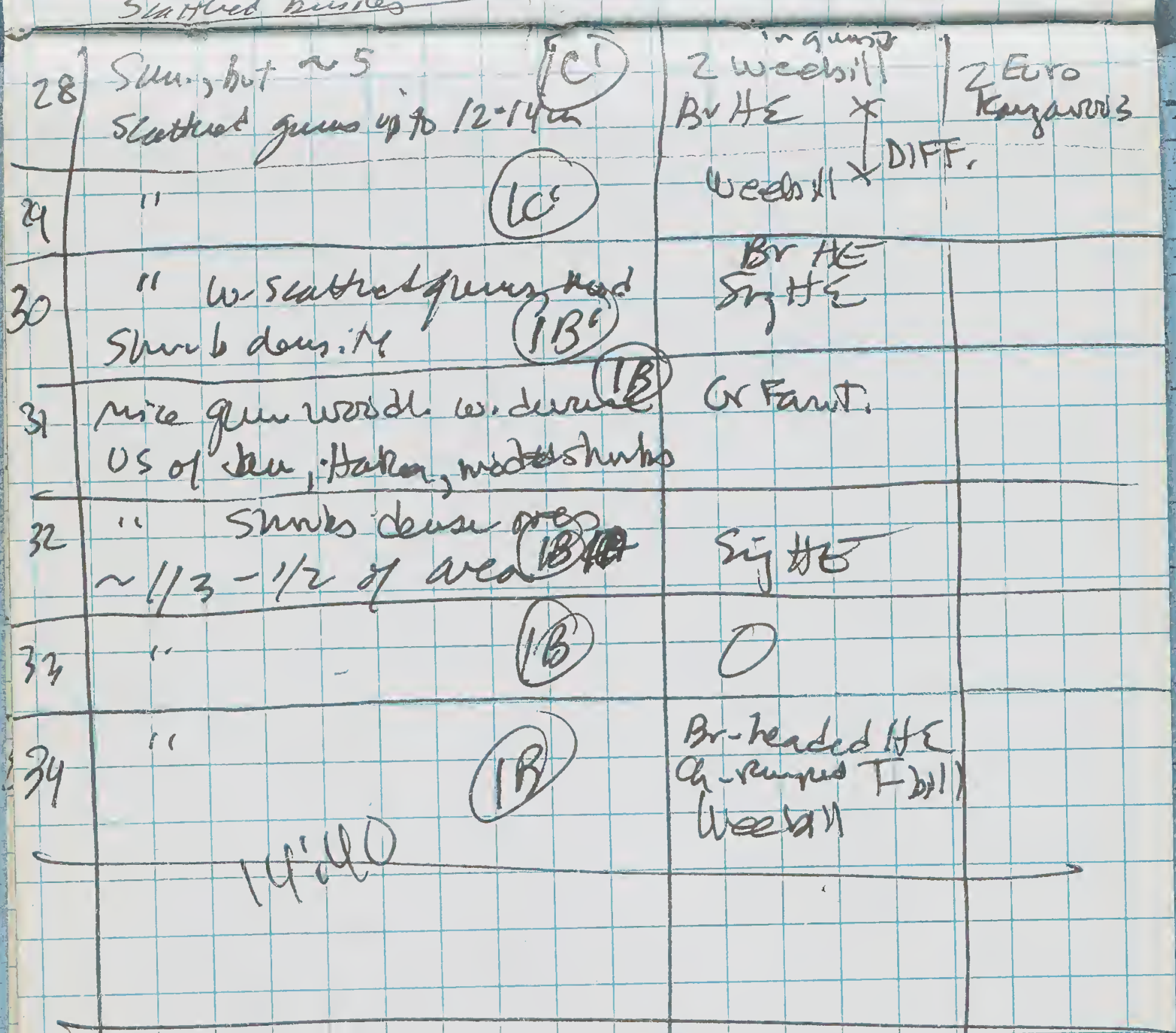

3) dula

motted or and \& Reel whisten hr. PT. when water pipe cuBs auray f. E. - F was W. 07 re, $q E$, but mey $\sim 100 \mathrm{~m}$ apart. also 4 w. Waubla, 3 fundalote, Y-RT-bill, Mutga Pay,grtan Prol Br Falcons desplay in lovi and laudin in rres es of rd 
To688. Durokoppun west ple, mild. 1:55 Ede Surverp

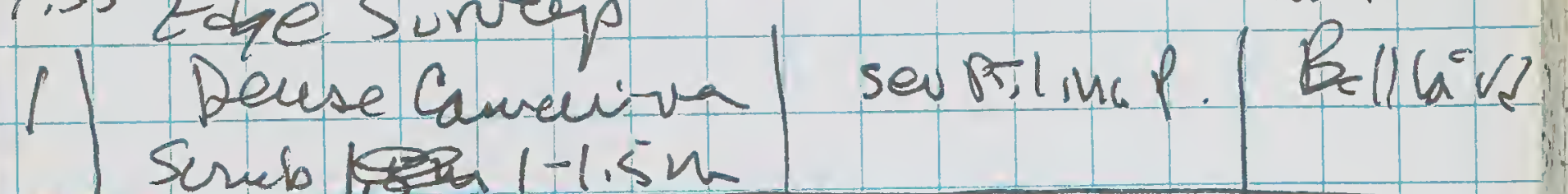
Scrub $1-1.5 \mathrm{~h}$

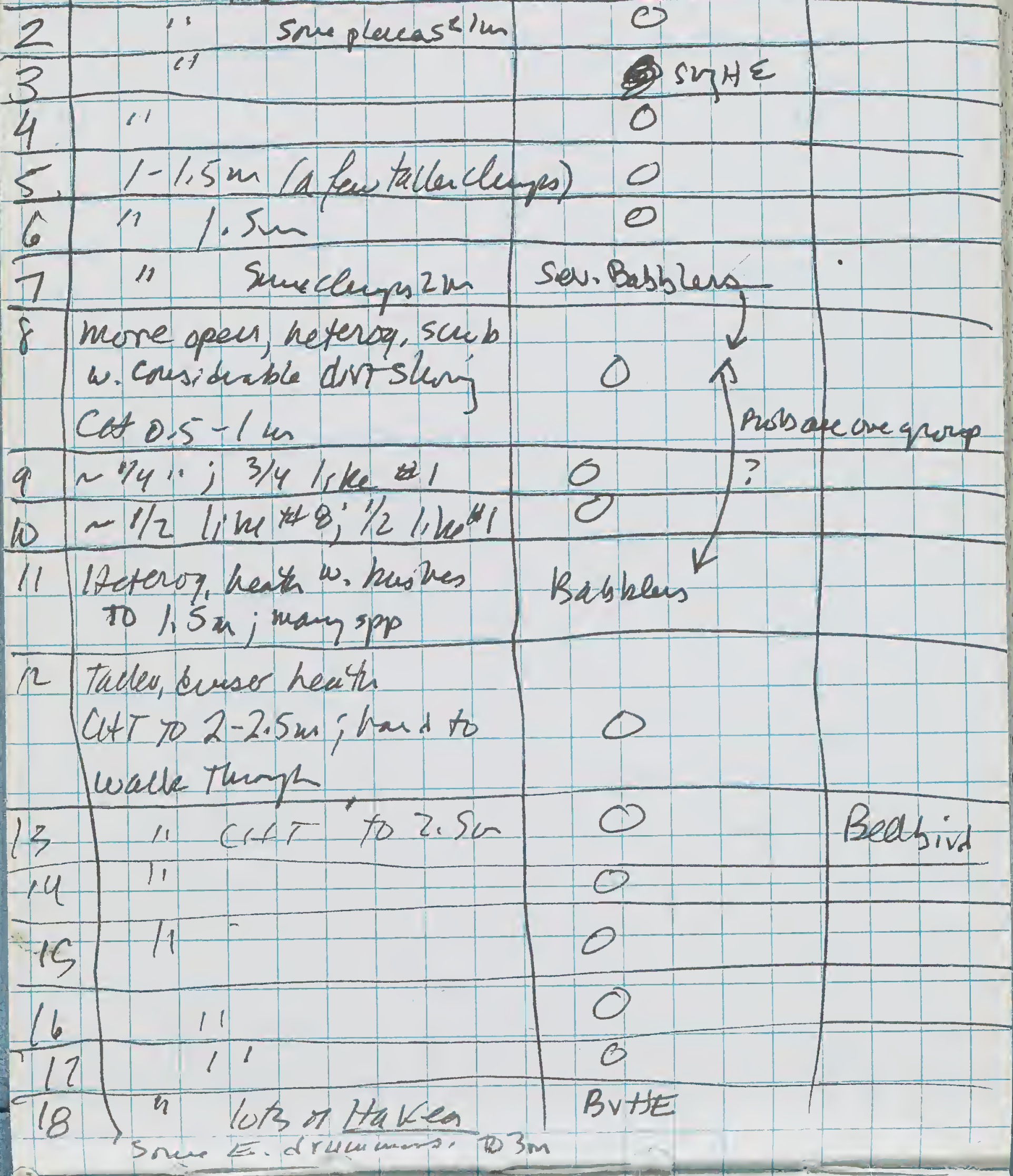

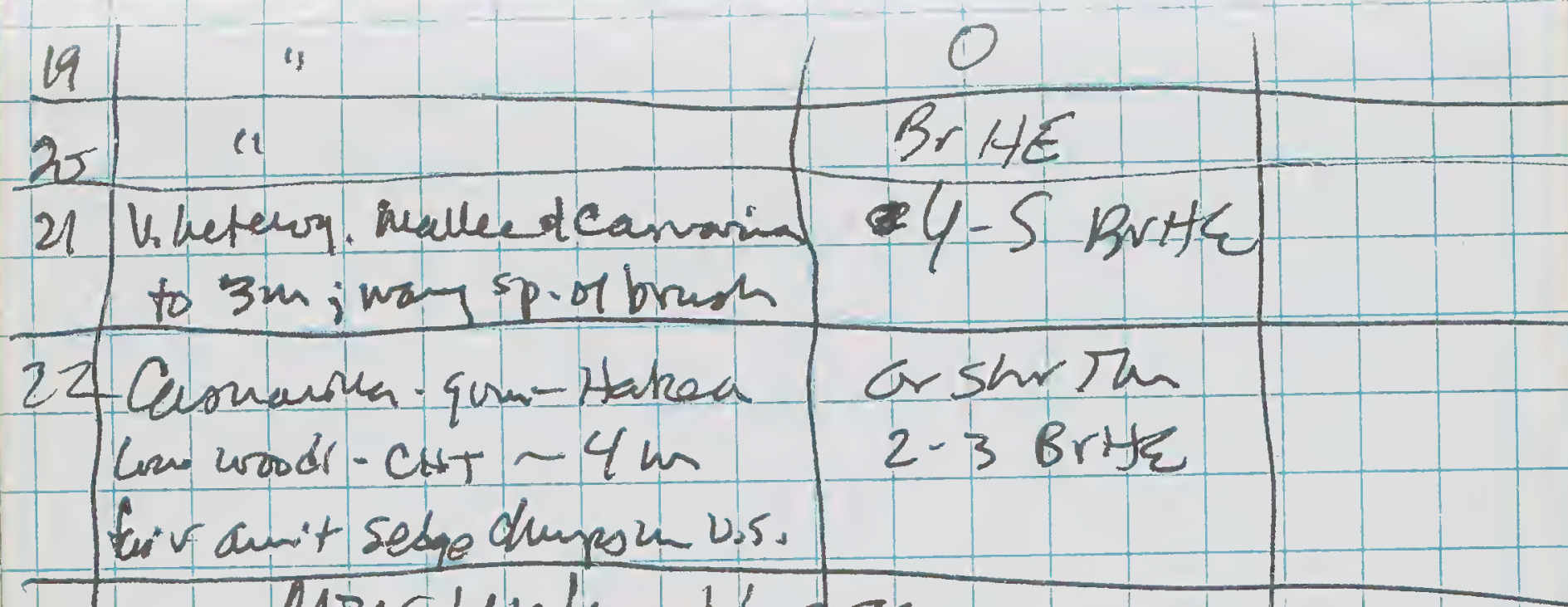

24

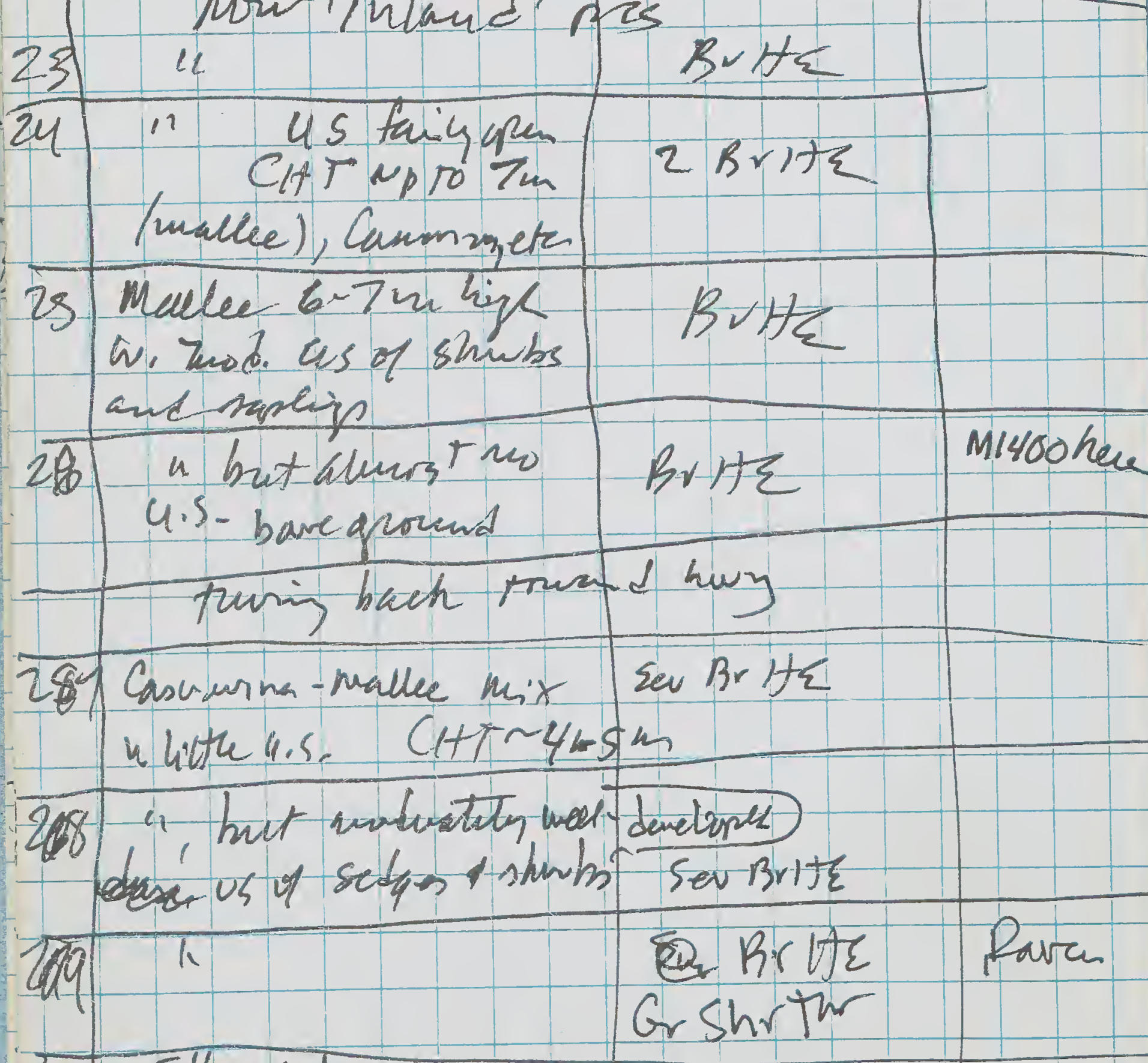

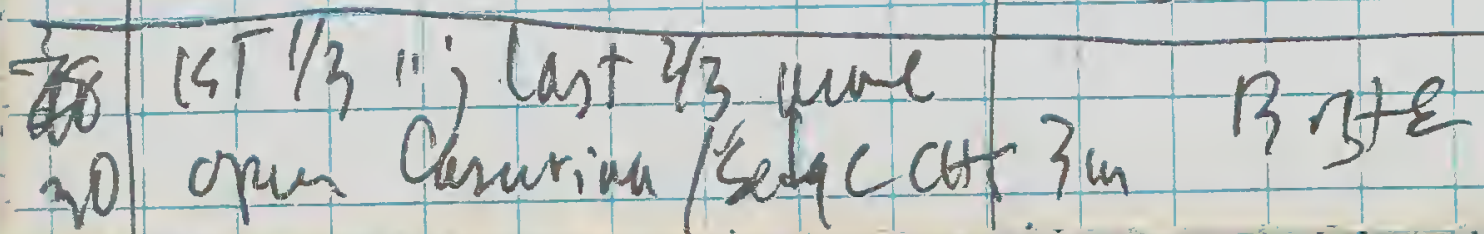


30) Patchy shrub/steppe ul

3 Htae clemps of Cascrarra (to Hakea (blovin) imotis tower shem, \& B of buude/seg

$2-2.5 m)$ 2 Bdte Beelsid

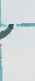

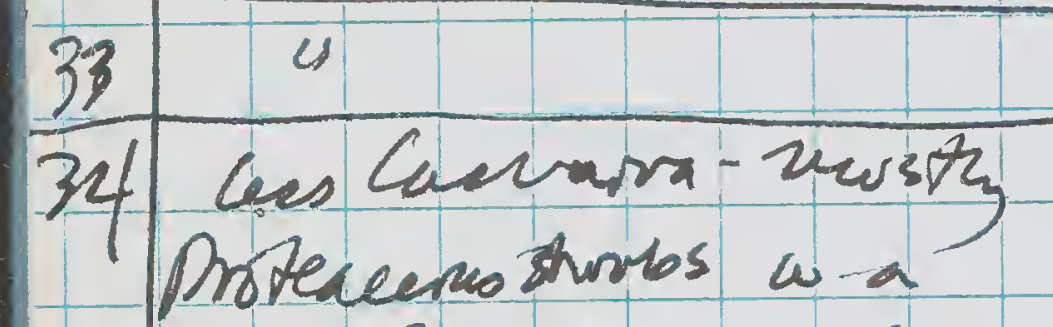
Kaw E Irumuma:; lots of sitqe elups

35 Tanly anse wallee tam. a. Sarubling us of hearin spe (tarly denas 36 1/2" i ind hanf bure, nure opea bresh/sedge $C+T 1.5 \mathrm{~m}$

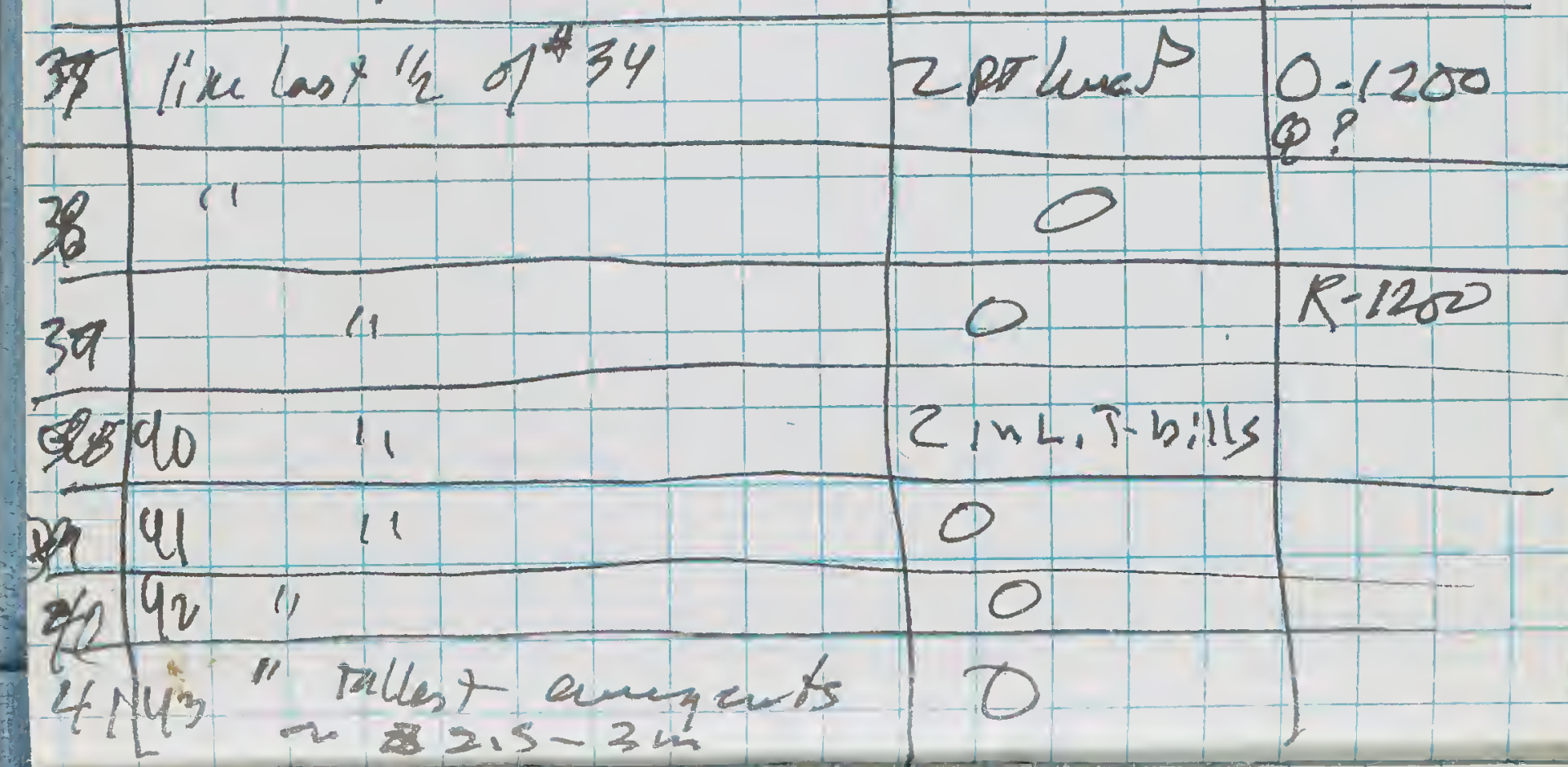

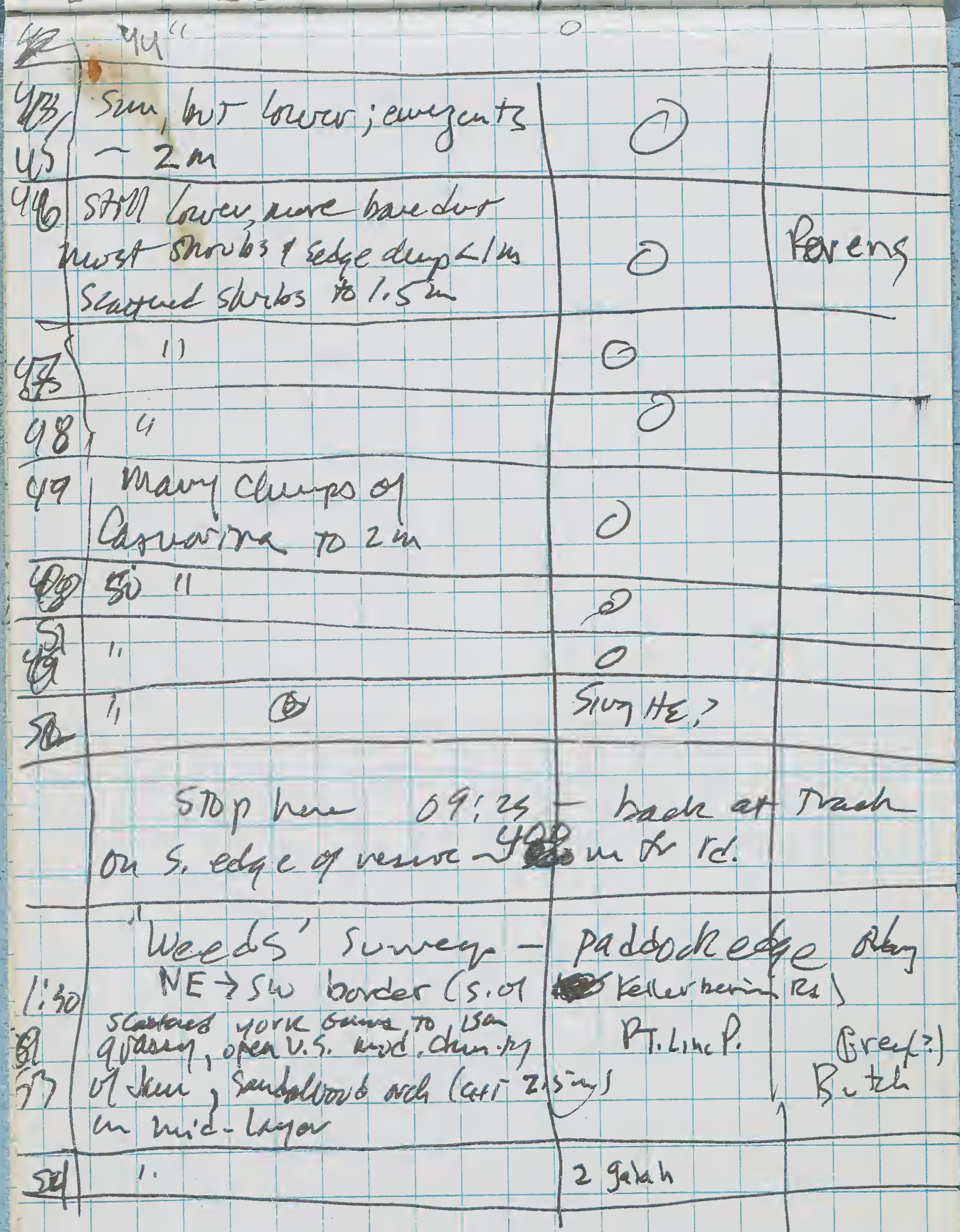

85) 1 t mue ogeen

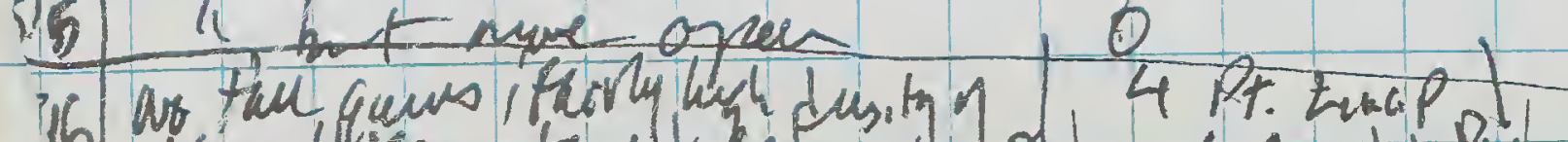

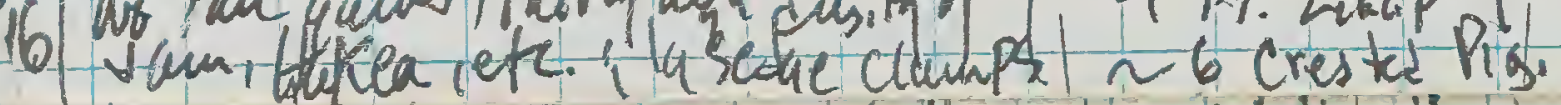
will! an 
20896 dito

0

80 a 1 t 0

Sa Scutued tae York $9 \mathrm{~mm}$ ius modure'ordi' rype, ereeth

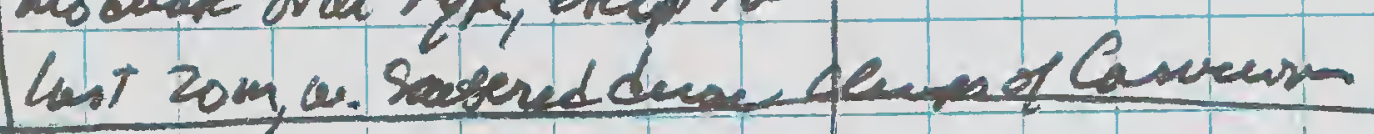

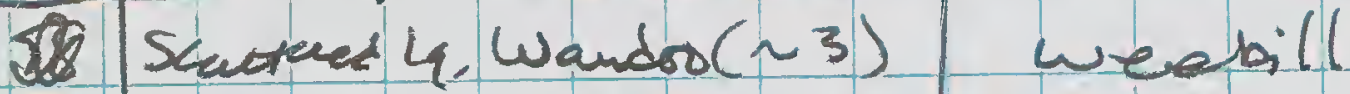

10 aver 'archard' of Jaupltaka

60 CoBol trast in 4.5.

Il Sun, but so tore peese

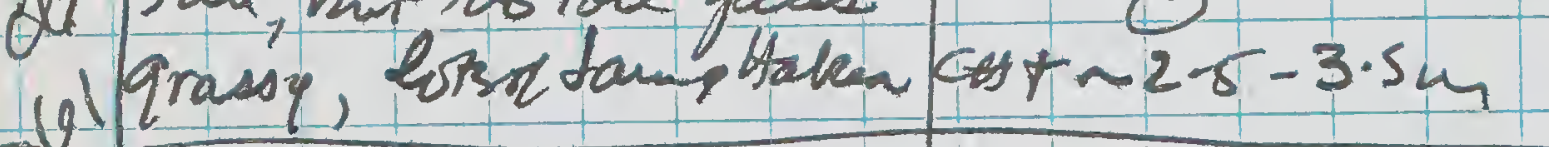

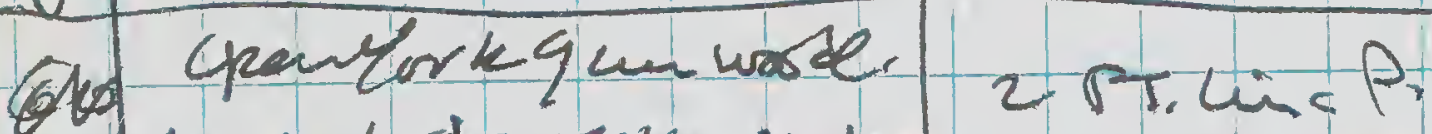

(N) W. Aud-stryozuan, sake

67 lith 459

CPt " (smallat.

1 nore-genimplaces

65 ditto, but 2 tree

1 Yivk jums

\&6 like 59

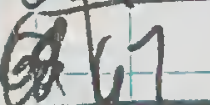

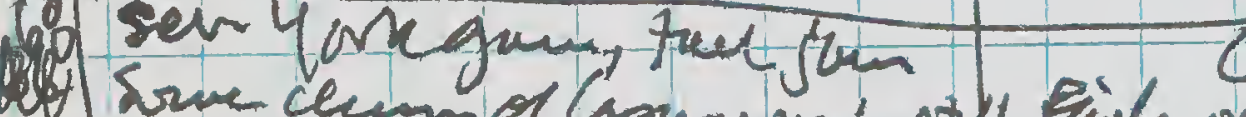

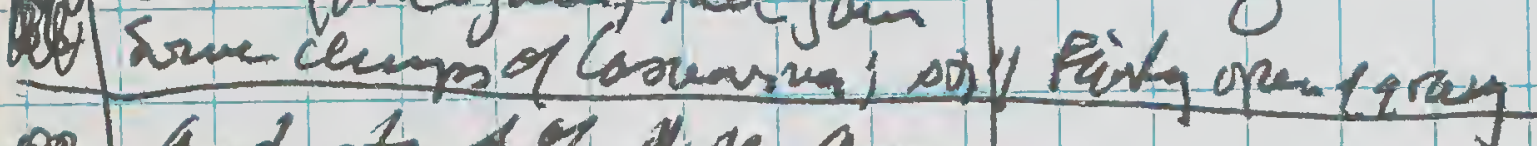

12 God stand of yok gum

9) Tor $12 \mathrm{~m}$; spueses of bou

6910

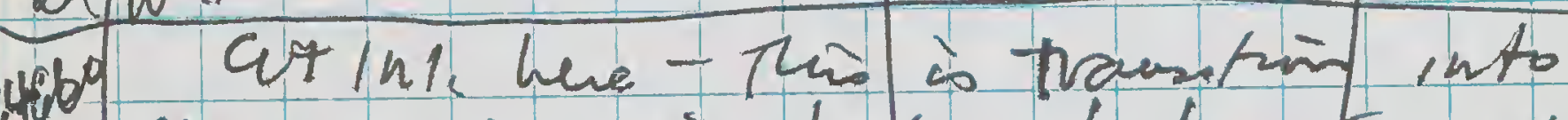

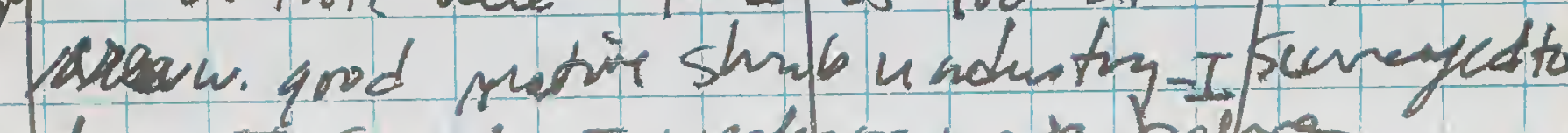
herethom th sw la t weeter weat bestore

STr. Pastalde-gleaing 'Needles' of Cosuariva $15 \mathrm{~m}$ up us ovita caupis r koviz "/4"keliy

R.e Robun vest w. 3 eq90 - in tork of dead Jam 1.5 m of anowd

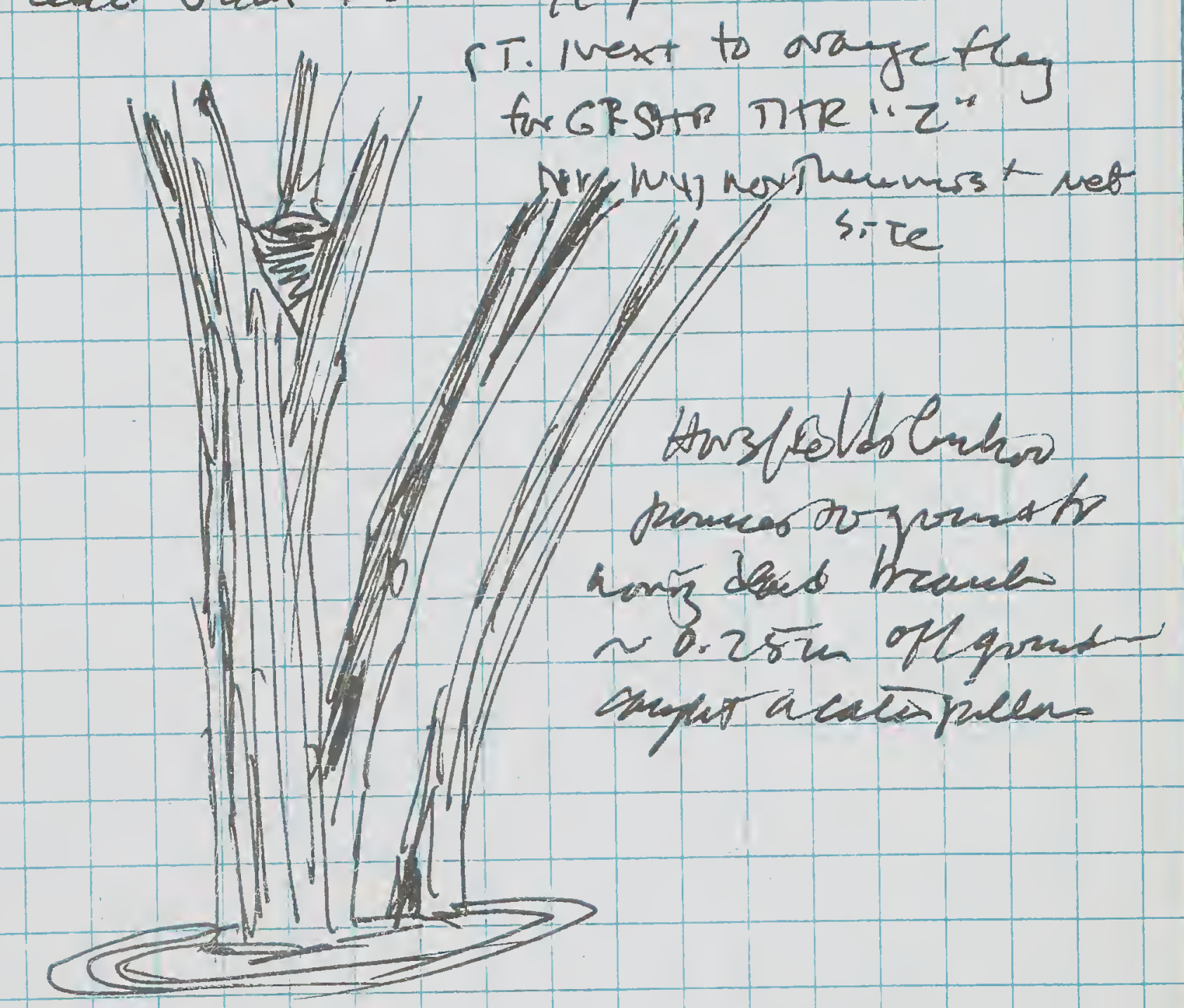




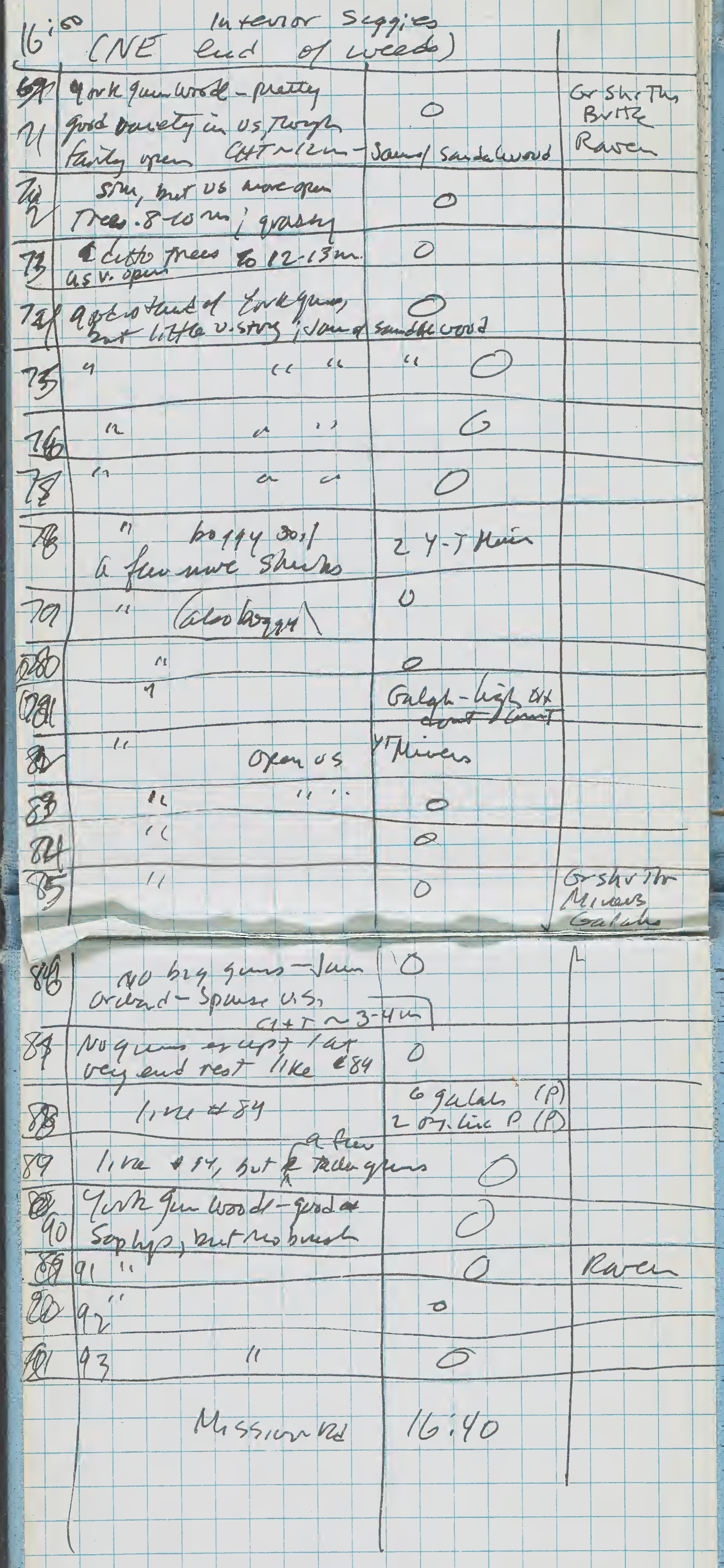



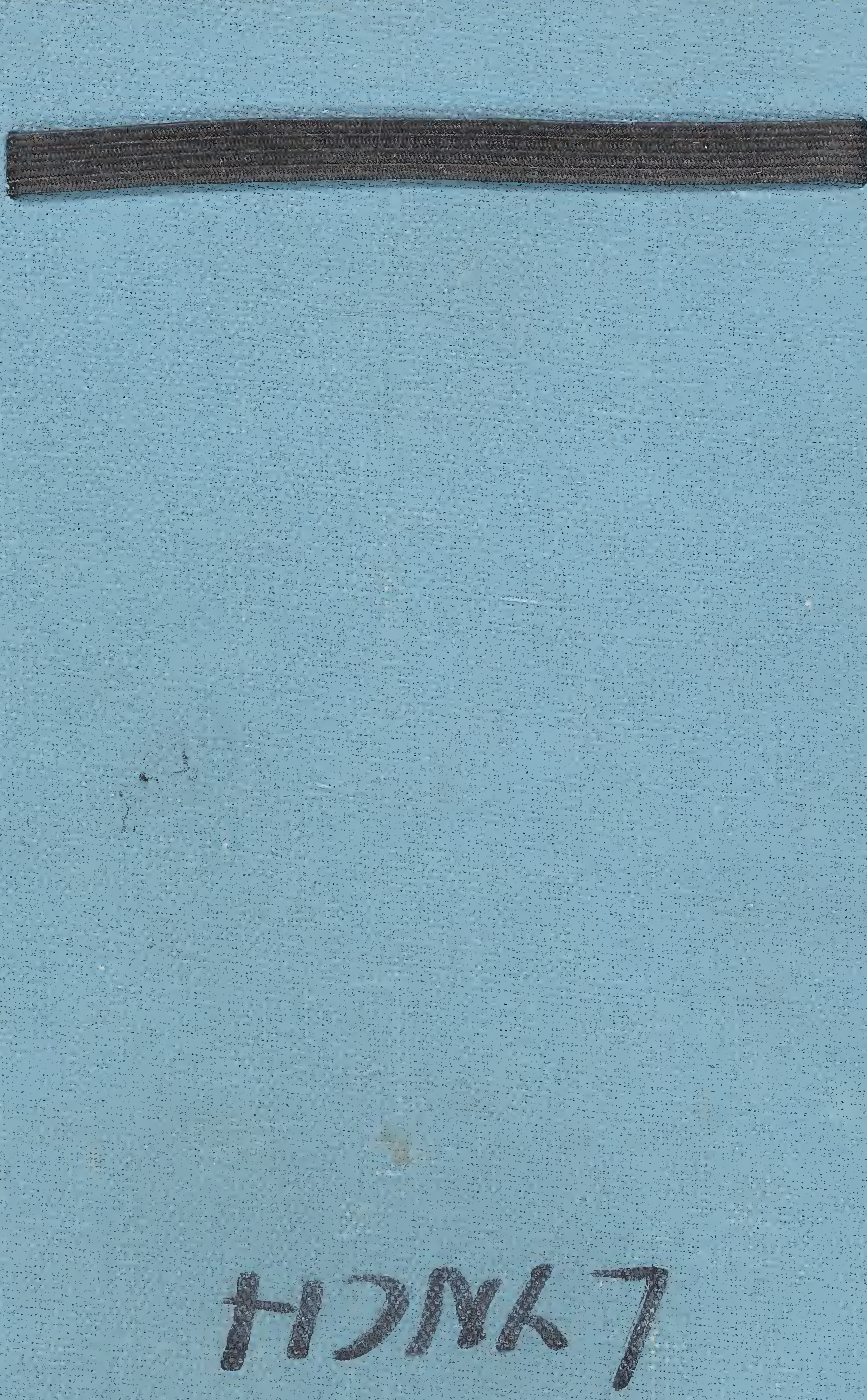